Florida International University FIU Digital Commons

$11-4-2016$

\title{
Carotenoids and Fatty Acids in Early Lactation: A Study of a Peruvian Population
}

Vanesa Mendez

vmend008@fiu.edu

DOI: $10.25148 /$ etd.FIDC001215

Follow this and additional works at: https://digitalcommons.fiu.edu/etd

Part of the Analytical Chemistry Commons, and the Dietetics and Clinical Nutrition Commons

\section{Recommended Citation}

Mendez, Vanesa, "Carotenoids and Fatty Acids in Early Lactation: A Study of a Peruvian Population" (2016). FIU Electronic Theses and Dissertations. 3007.

https://digitalcommons.fiu.edu/etd/3007 


\section{FLORIDA INTERNATIONAL UNIVERSITY}

Miami, Florida

\section{CAROTENOIDS AND FATTY ACIDS IN EARLY LACTATION: A STUDY OF A PERUVIAN POPULATION}

A dissertation submitted in partial fulfillment of the

requirements for the degree of

DOCTOR OF PHILOSOPHY

in

CHEMISTRY

by

VANESA MENDEZ 
To: Dean Michael R. Heithaus

College of Arts, Sciences and Education

This dissertation, written by Vanesa Mendez, and entitled Carotenoids and Fatty Acids in Early Lactation: A Study of a Peruvian Population, having been approved in respect to style and intellectual content, is referred to you for judgment.

We have read this thesis and recommend that it be approved.

$\begin{array}{r}\text { Richard Bone } \\ \hline \text { Drancisco Fernandez-Lima } \\ \hline \text { Fenfei Leng } \\ \hline \text { John T. Landrum, Major Professor }\end{array}$

Date of Defense: November 4, 2016

The dissertation of Vanesa Mendez is approved.

Dean Michael R. Heithaus

College of Arts, Sciences and Education

Andrés G. Gil

Vice President for Research and Economic Development and Dean of the University Graduate School

Florida International University, 2016 


\section{DEDICATION}

I dedicate this dissertation to my mother for being an example to me and for her unconditional love. 


\section{ACKNOWLEDGMENTS}

First, I would like to thank my advisor Dr. John Landrum for his guidance, support, patience, and for all the time that he had invested helping, and encouraging me during these years; in particular for keeping a positive attitude at all times. I am glad that I had you as my mentor. I would like to thank the members of my committee Dr. Richard Bone, Dr. David Chatifield, Dr. Fenfei Leng, and Dr. Francisco Fernandez-Lima for their time and helpful feedback on my research. I also, would like to thank Dr. Anthony DeCaprio for allowing us to use his research facilities and Professor Ramon Gomez for his help during my research.

I would like to thank my friends from FIU and lab mates for all their support and friendship; it has been a real pleasure to know you all and had the opportunity to spend time with you during the past years.

A special thank you to my parents from the bottom of my heart, for supporting and encouraging me to pursue a career far from our homeland. Also, I am sincerely grateful for my family in Peru and in Miami. I am happy and proud to have each of you in my life.

I wish to express my endless gratitude to my mother, Amanda, and Malu. They have been the best cheer leaders during this journey. I feel blessed for your care, love and support.

Thank you God for always guiding my footsteps. 


\title{
ABSTRACT OF THE DISSERTATION
}

\section{CAROTENOIDS AND FATTY ACIDS IN EARLY LACTATION: A STUDY OF A PERUVIAN POPULATION}

\author{
by \\ Vanesa Mendez \\ Florida International University, 2016 \\ Miami, Florida \\ Professor John T. Landrum, Major Professor
}

Lipid soluble carotenoids are micronutrients present in human milk that serve as precursors of vitamin A and also play an important role protecting cells from damage arising from photooxidative processes and reactive oxygen species. Fatty acids comprise about 3-5\% of human milk and are mainly present as triglycerides. They are a major energy source for the infant and are necessary to support cell growth required for normal development and maturation of critical organs. Transport of carotenoids into milk has been little studied and there has been no previous investigation of the relationship of carotenoid transport with that of individual fatty acid secretion into milk.

In the present study, levels of the carotenoids, lutein, zeaxanthin, $\beta$-cryptoxanthin, and $\beta$-carotene, in maternal serum, infant cord blood, and milk obtained from 74 Peruvian mothers were measured by HPLC methods. The fat content and fatty acid profile of maternal milk were determined by GC-FID and confirmed by GC-MS. Twenty nine fatty acids were identified and quantified after conversion to methyl esters. 
Statistical analysis was employed to investigate potential trends and relationships among the carotenoids in all three fluids as well as between carotenoids and fatty acids present.

Concentrations of lutein in maternal serum and milk as well as maternal serum and infant cord blood were highly correlated $(r=0.43, \mathrm{p}<0.03)$ and $(\mathrm{r}=0.40, \mathrm{p}<0.05)$, respectively. Body mass index was inversely correlated with the concentration of provitamin A carotenoids in milk and serum. Carotenoid concentrations declined during the initial weeks of lactation and did not significantly correlate with fat content which remained constant. Concentrations of essential n-3 and n-6 fatty acids were found to be in the upper quartile among others populations studied worldwide. An inverse correlation between lutein and the concentrations of linoleic acid $(\mathrm{r}=-0.432, \mathrm{p}<0.02)$ and $\alpha$-linoleic acid $(\mathrm{r}=-0.435, \mathrm{p}<0.02)$ present in transitional milk was seen. Moreover, secretion of carotenoids into milk shows a positive correlation with a small number of fatty acids that are metabolically produced within the mammary duct. A competitive relationship between the transport of these essential polyunsaturated fatty acids is hypothesized to play a role in the secretion of early milk but not that of mature milk. 


\section{TABLE OF CONTENTS}

CHAPTER

PAGE

CHAPTER I: Carotenoid properties, functions and bioactivity influence in the human

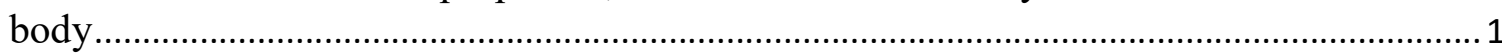

1.1. Carotenoid Biosynthesis............................................................................ 1

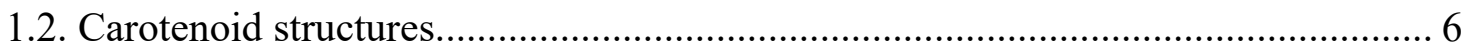

1.3. Physical and Chemical properties ............................................................. 10

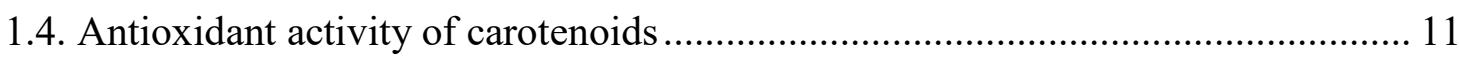

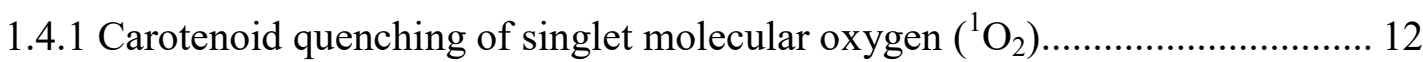

1.5. Bioactivity and disease prevention.......................................................... 14

1.6. Transport and distribution of carotenoids in the human body ........................... 15

1.7. Pro-vitamin A and non-pro-vitamin A carotenoids......................................... 18

1.7.1. $\beta$-carotene, $\alpha$-carotene, and $\beta$-cryptoxanthin (pro-vitamin A carotenoids) ..... 18

1.7.2 Lutein and Zeaxanthin (non-pro-vitamin A carotenoids)............................. 21

CHAPTER II: Carotenoids in maternal serum and cord blood..........................................26

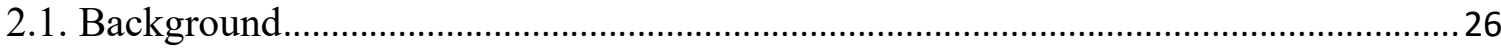

2.1.1. Analysis of carotenoids in human serum ................................................ 26

2.1.2. Carotenoids in maternal plasma and infant cord blood ................................ 27

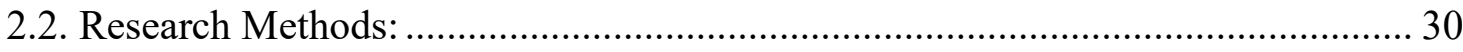

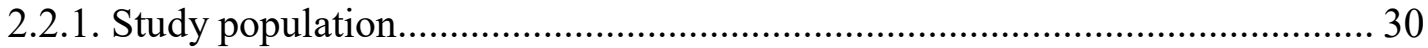

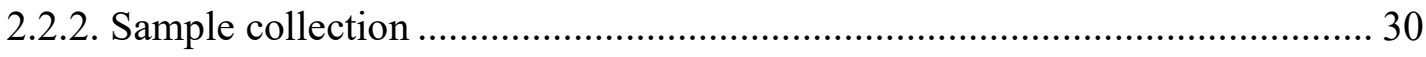

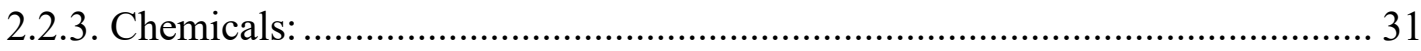

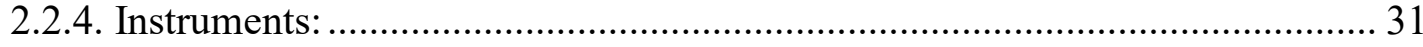

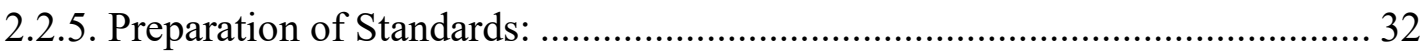

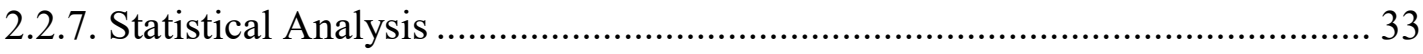

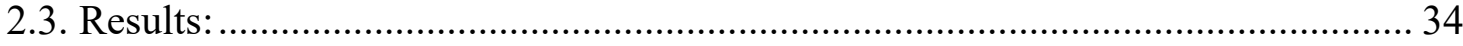

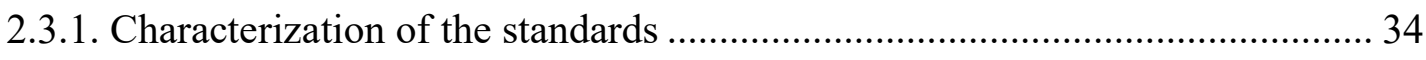

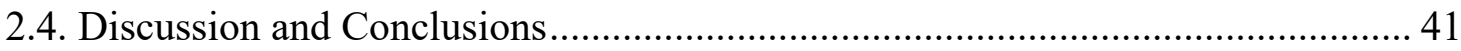


CHAPTER III: Fatty acids in human milk ................................................................. 46

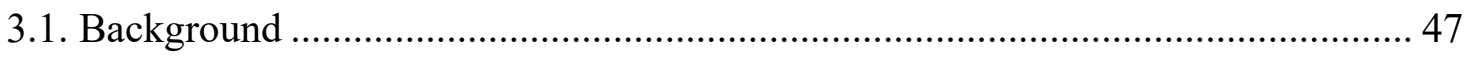

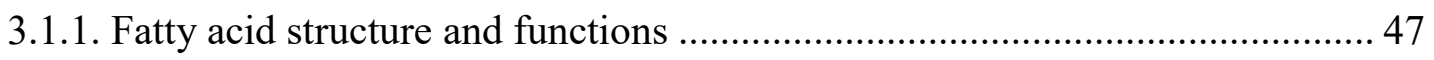

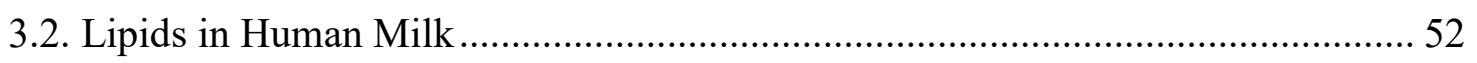

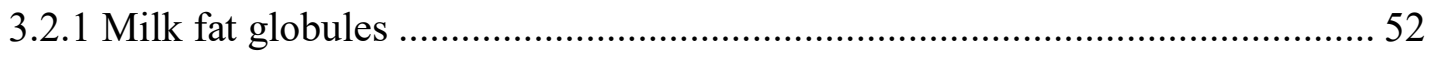

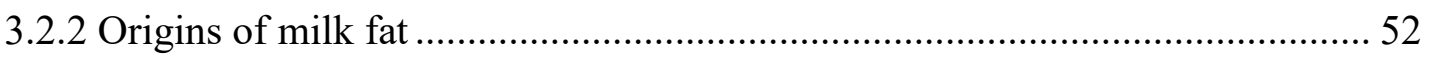

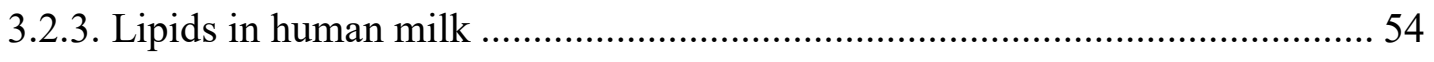

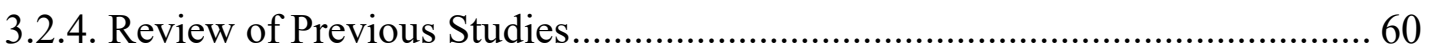

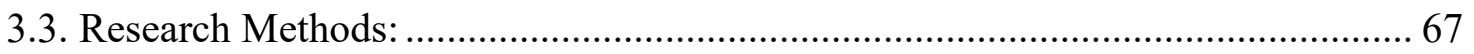

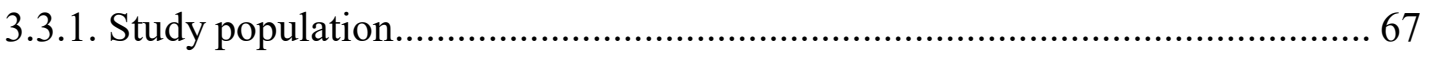

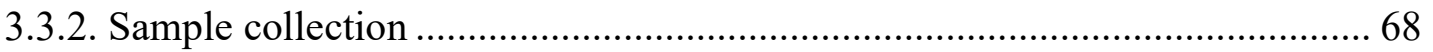

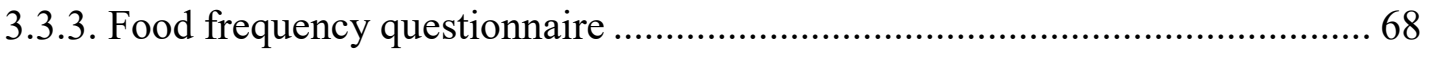

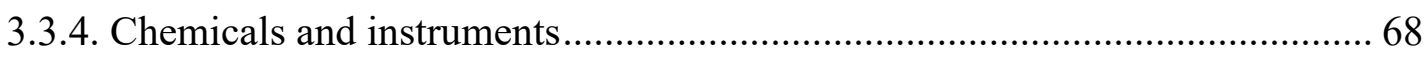

3.3.5. Determination of FAME in human milk .............................................. 70

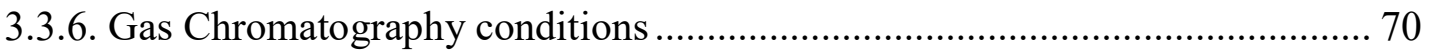

3.3.7. Identification and quantification of fatty acids........................................ 70

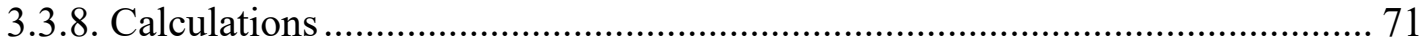

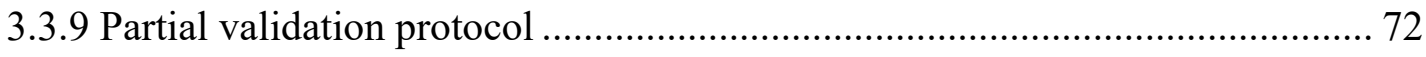

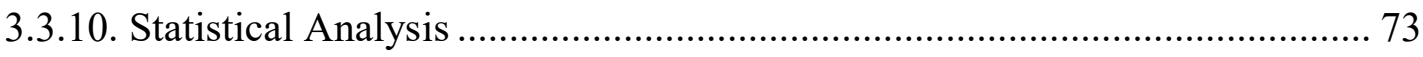

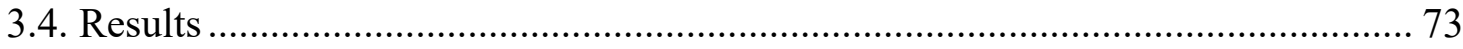

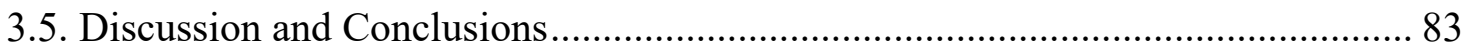

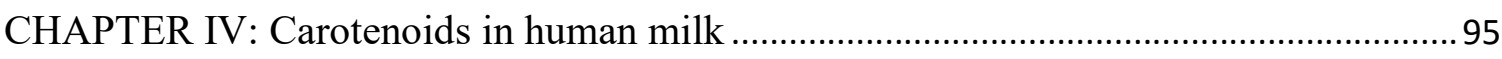

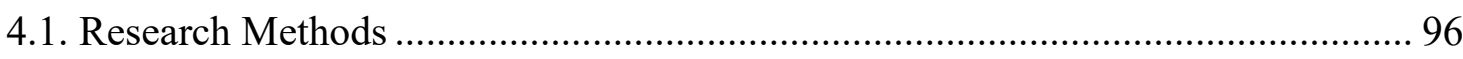

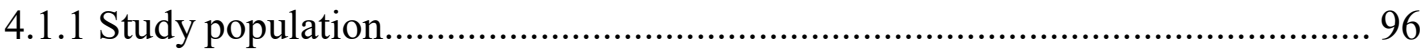

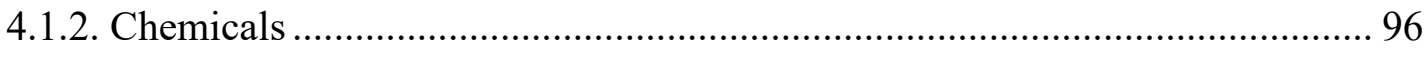

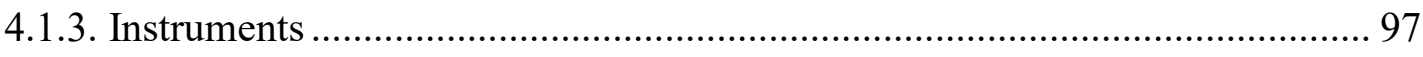

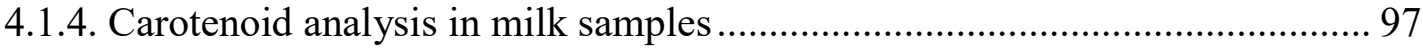

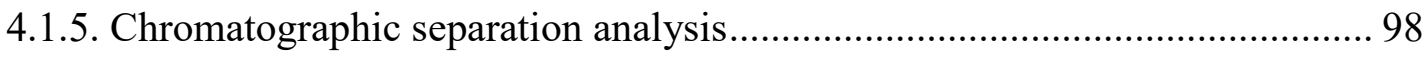

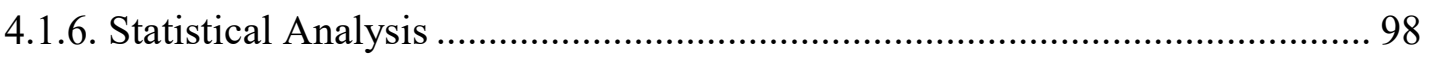

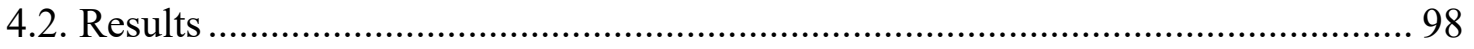




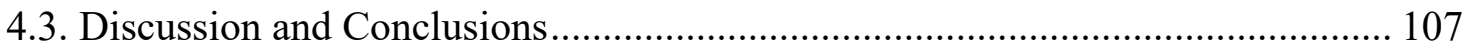

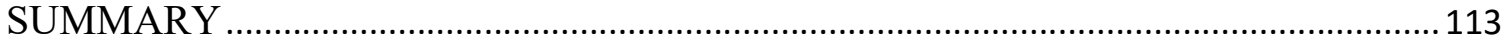

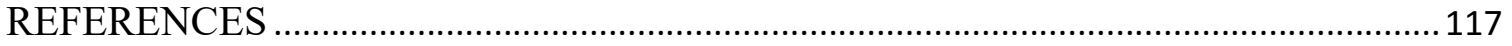

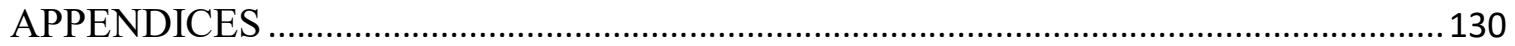

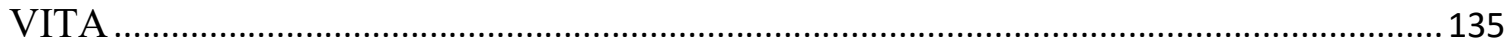




\section{LIST OF TABLES}

TABLE

PAGE

Table 1. End-Group Designations of Carotenes. 8

Table 2. Carotenoids, functions and effects associated with prevention of human diseases and optimization of human health (taken from Takana et al. [26]). .16

Table 3. Studies showing carotenoid levels $(\mu \mathrm{mol} / \mathrm{L})$ in maternal plasma though pregnancy and cord blood of new born infants.

Table 4. Results from the analysis of carotenoids in cord blood for all subjects, amounts in $\mu \mathrm{mol} / \mathrm{L}$

Table 5. Results of analysis of carotenoids in mother's serum for all subjects, amounts in $\mu \mathrm{mol} / \mathrm{L}$ 38

Table 6. Correlations (r) of carotenoids in serum 39

Table 7. Correlation between BMI and pro-vitamin A carotenoids in maternal serum. ...41

Table 8. Lutein and zeaxanthin concentrations ( $\mu \mathrm{mol} / \mathrm{L})$ in cord blood according to newborn's sex.

Table 9. Average carotenoids levels $(\mu \mathrm{mol} / \mathrm{L})$ in mother's serum.

Table 10. Average carotenoids levels $(\mu \mathrm{mol} / \mathrm{L})$ in cord blood.

Table 11. Carotenoid concentrations $(\mu \mathrm{mol} / \mathrm{L})$ for maternal and cord blood in paired samples.

Table 12. Multiple functions of the major nutrients of human milk (adapted from [77] p. 70). LC-PUFA = long chain polyunsaturated fatty acids; FFA = free fatty acids, produced from triglycerides during fat digestion in the stomach and intestine.

Table 13. Factors associated with changes in the total lipid content of human milk (adapted from [75] p. 1254).

Table 14. Lipid class composition during lactation (adapted from [92]). Amounts in wt $\%$, total lipid $\%$ in mean \pm SEM. 
Table 15. Distribution (\%) of essential fatty acids in the glycerol structure in colostrum and mature milk (adapted from [96] p. 270). LA = linoleic acid, ALA $=\alpha-$ linoleic acid, $\mathrm{AA}=$ arachidonic acid, $\mathrm{DHA}=$ docosahexaenoic acid

Table 16. List of studies grouped according to their geographic location, country and first author of the citation.

Table 17. Amounts of fatty acid methyl esters $(\mu \mathrm{g})$ used to prepare calibration curves relative to the internal standard. .72

Table 18. Retention times and response factors for FAMES calculated from the standard mixture run by GC-FID. .76

Table 19. Repeatability measurements from five replicates (mean $\pm \%$ RSD). .78

Table 20 . Total $\%$ of fat in all milk samples during $2 \mathrm{nd}$, 3rd and 4 th week after delivery.

Table 21 . Fatty acid composition of transitional and mature milk $(\% \mathrm{w} / \mathrm{w})$ in all subjects. .80

Table 22 . Fatty acid composition of mother's milk ( $\% \mathrm{w} / \mathrm{w})$ for the same subjects. .81

Table 23 . Total $\%$ of fat in milk samples during week 2,3 and 4 th after delivery for the same subjects. .82

Table 24. Food frequency intake. .83

Table 25. FAs with a significant difference between weeks 2 and 4 of lactation .83

Table 26. Total amounts $(\% \mathrm{w} / \mathrm{w})$ variation during the first month by type of FA..........85

Table 27. Elaidic acid in human milk in different countries $(\% \mathrm{w} / \mathrm{w})$.

Table 28. DHA content ( $\% \mathrm{w} / \mathrm{w})$ in human milk of US women in studies conducted from 1992-2012. Taken from Brenna et al.[126] and Martin et al.[114].

Table 29. FA concentrations (mg/L) from Peruvian mothers in mature milk (4th week of lactation). $\mathrm{N}=74$.

Table 30. Human milk FA concentrations (mg/L). Taken from Yuhas et al.[104] .93 
Table 31. Carotenoid concentrations in human milk during the 2 nd week of lactation (transitional milk, W2)

Table 32. Carotenoid concentrations in human milk during the 3rd week of lactation (mature milk, W3).

Table 33. Carotenoid concentrations in human milk during the 4th week of lactation (mature milk, W4).

Table 34. Carotenoid concentrations in maternal serum and milk during the 4th week (W4) after delivery.

Table 35. Longitudinal, subject by subject comparison of carotenoid concentrations and total fatty acid content between weeks. Values reported are mean concentrations (mean $\pm \mathrm{SEM}$ ) in $\mu \mathrm{mol} / \mathrm{L}$

Table 36. Carotenoid concentrations (mean \pm st.dev., $\mu \mathrm{mol} / \mathrm{L}$ ) in mature maternal milk (W4) grouped according to the subject's body mass index....

Table 37. Pearson correlation coefficients obtained from the subject by subject comparison of carotenoid concentrations and individual fatty acid concentrations in transitional milk (W2).

Table 38. Pearson correlation coefficients obtained from the subject by subject comparision of carotenoid concentrations and individual fatty acid concentrations in in mature milk (W3 and W4). 


\section{LIST OF FIGURES}

FIGURE

PAGE

Figure 1. Formation of IPP from MVA …..............................................................2

Figure 2. General pathway for the formation of phytoene [7]. The arrows on the side represent the enzymes involved. GGPS: geranylgeranyl diphosphate synthase. PSY: phytoene synthase. 3

Figure 3. General pathway for the formation of lycopene [7]. The arrows on the side represent the enzymes involved. PDS: phytoene desaturase. ZDS: $\zeta$-carotene desaturase.

Figure 4. Cyclization of lycopene. Enzymatic conversions are shown by arrows with the enzymes responsible. LCY- $\beta$ : lycopene beta-cyclase; LCY- $\varepsilon$, lycopene epsiloncyclase; CRH- $\beta, \beta$-carotene hydroxylase; $\mathrm{CRH}-\varepsilon$, epsilon-carotene hydroxylase [8].........5

Figure 5. Basic mechanism for biosynthesis of $\varepsilon$ and $\beta$-rings in carotenoids...................6

Figure 6. Carotenoid structure. A) Isoprene unit. B) C40 Structure of Lycopene..............7

Figure 7. Carotene numbering system. .............................................................

Figure 8. Carotene ( $\beta$-carotene) and Xanthophylls (lutein and zeaxanthin).....................8

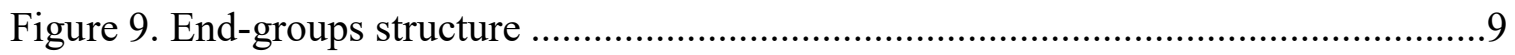

Figure 10. Electronic absorption spectra of different carotenoids (extracted from tomatoe in $80 \%$ acetone/diethyl ether). Taken from [13] ........................................ 11

Figure 11. Diagram of the electronic states of the $\mathrm{O}_{2}$ molecule....................................12

Figure 12. Examples of provitamin A carotenoids .................................................... 18

Figure 13. Central and eccentric cleavage of $\beta$-carotene (taken from Harrison [32])......19

Figure 14. Major components of the macular pigment............................................21

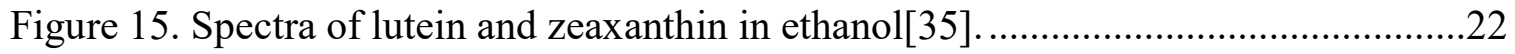


Figure 16. A) Human retina, taken from:

http://www.gene.com/gene/products/education/tgr/eye-health-glossary.html. B)

Frozen section through the fovea of a rhesus monkey, taken from the Snodderly Lab, http://www.sbs.utexas.edu/SnodderlyLab/galler.

Figure 17. UV-visible spectra of standards used for analysis and identification.

A)Monopentyl lutein and B) $\beta$-cryptoxanthin. .34

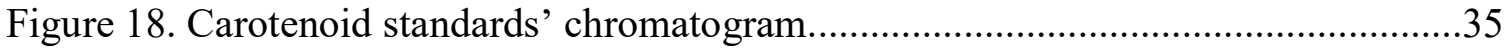

Figure 19. Example of mother's serum chromatogram ..................................................35

Figure 20. A typical chromatogram of the carotenoids extracted from cord blood............36

Figure 21. Correlation of lutein in maternal serum and cord blood $(\mathrm{r}=0.395, \mathrm{p}=0.046$, $\alpha=0.05)$

Figure 22 Correlation of lutein and zeaxanthin in (A) cord blood $(\mathrm{r}=0.69, \mathrm{p}<0.001)$

(B) maternal serum $(\mathrm{r}=0.81, \mathrm{p}<0.001, \alpha=0.05)$.

Figure 23 Correlation of BMI to maternal serum of (A) $\beta$-cryptoxanthin $(r=-0.28$, $\mathrm{p}=0.02)(\mathrm{B}) \beta$-carotene $(\mathrm{r}=-0.26, \mathrm{p}=0.055, \alpha=0.05)$

Figure 24. The structure of linoleic acid (LA), short formula 18:2n-6 .............................48

Figure 25. The structure of a saturated fatty acid, stearic acid (18:0).

Figure 26. The structures of the monounsaturated fatty acids of oleic acid (18:1n-9)

(a) the trans structure, elaidic acid and (b) the naturally occurring cis structure. .50

Figure 27. The structures of polyunsaturated fatty acid, (a) $\alpha$-linolenic acid (ALA, 18:3n-3), (b) eicosapentaenoic acid (EPA, 20:5n-3), and (c) docosahexaenoic acid (DHA, 22:6n-3).

Figure 28. The stereospecific number of the substitution of fatty acids in triacylglycerols

Figure 29. Metabolic conversion of linoleic acid ( $\mathrm{n}-6$ series) and $\alpha$-linolenic acid (n-3 series) to form the long-chain polyunsaturated fatty acids (adapted from [97] p.7). .57

Figure 30. Fatty acids concentrations in human milk summarized by geographic regions of the world a) SFA: palmitic acid, mean $=21.5 \% \mathrm{w} / \mathrm{w}$; b) MUFA: oleic acid, mean $=29.8 \%$. w $/ \mathrm{w}$. 
Figure 31. Essential PUFAs summarized by geographic regions over the world A)

Linoleic acid, mean $=13.5 \% \mathrm{w} / \mathrm{w}$. B) $\alpha$-linoleic acid, mean $=1.2 \% \mathrm{w} / \mathrm{w}$. .63

Figure 32. Long Chain-PUFAs by country. A) AA, mean $=0.51 \% \mathrm{w} / \mathrm{w}$ and median $=0.46 \% \mathrm{w} / \mathrm{w}$. B) DHA, mean $=0.32 \% \mathrm{w} / \mathrm{w}$ and median $=0.23 \% \mathrm{w} / \mathrm{w}$. .65

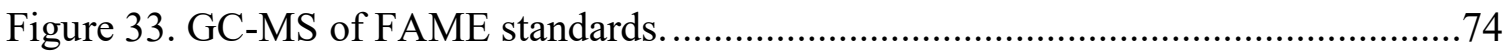

Figure 34. Example of a GC-MS chromatogram of a maternal milk sample...................75

Figure 35. Calibration curves for FAMEs relative to the internal standard with different chain lengths (a) $\mathrm{C} 12$, (b) $\mathrm{C} 16$, (c) $\mathrm{C} 18$, and (d) C20..................................77

Figure 36. Calibration curve for to the internal standard C13 ...................................79

Figure 37 . Total amounts $(\% \mathrm{w} / \mathrm{w})$ variation during the first month by type of FA..........85

Figure 38. Histogram, all countries including Peru, for A) Palmitic acid, Mean $=$ $21.77 \%, \mathrm{~N}=29$. B) Oleic acid, Mean $=30.3 \%, \mathrm{~N}=31$. Blue dashed line represents the mean of all countries. Red square represents Peru.

Figure 39. Histogram, all countries reviewed, for A) Linoleic acid, Mean $=14.08 \%$, Median $=13.8 \%, N=33$. B) $\alpha$-linoleic acid, Mean $=1.12 \%$, Median $=1.05 \%, N=33$. Blue dashed line represents the mean of all countries. Red square represents Peru.

Figure 40. LA:ALA ratios for different countries. Peru is represented in red. Dashed line represents the median of all countries $=13.6$.

Figure 41. Typical reversed-phase HPLC chromatogram of the carotenoid analysis of a sample of human milk.

Figure 42. Carotenoid concentrations (mean $\pm \mathrm{SEM}$ ) in $\mu \mathrm{mol} / \mathrm{L}$ during the first month post-partum (data from Table 25).

Figure 43. Comparison of pro-vitamin A carotenoids by BMI group (data from Table 26). Concentrations (mean \pm standard deviation)......

Figure 44. Composition of carotenoids in serum and mature milk during the 4th week post-partum.

Figure 45. Relation of essential fatty acids with lutein and zeaxanthin in transitional milk. 
Figure 46. Carotenoid concentration in maternal milk, serum, and cord blood.

Concentration (mean \pm S.E.M.). Serum, $n=74$; milk W2, $n=30$; milk W3, $n=28$; milk $\mathrm{W} 4, \mathrm{n}=74$; and cord blood, $\mathrm{n}=26$.

Figure 47. Lutein, zeaxanthin, $\alpha$-linoleic (ALA), and linoleic acid (LA) variations $($ mean \pm SEM) between transitional and mature milk...........................................115 
CHAPTER I: Carotenoid properties, functions and bioactivity influence in the human body

Carotenoids exist in nature as pigments in microorganisms and plants. They are synthesized in plants, fungi, bacteria and algae, therefore these compounds are considered essential for life since they actively participate in the process of photosynthesis [1]. Carotenoids are responsible for the coloration in many flowers, fruits and vegetables and they are also the principle pigments in various animals: fish, birds' feathers and crustaceans [2]. Humans cannot synthesize carotenoids but they can obtain them from their diet and use them as antioxidants. In early life, newborns acquire these micronutrients during lactation and they are important for their development. More than 750 of these compounds had been identified in nature [3], but only 33, including geometric isomers and metabolites, have been detected in human plasma tissues, and breast milk $[4,5]$.

\subsection{Carotenoid Biosynthesis $[6,7]$}

In plants, carotenoids are a family of pigments that participate in light harvesting and are essential for photoprotection against excess light. Carotenoids are synthesized in the plastids by enzymes that are encoded by the nucleus. Carotenoids are built from the biological isoprene precursor isopentenyl pyrophosphate (IPP). The first specific terpenoid precursor is mevalonic acid (MVA). Mevalonic acid is converted into IPP by means of a three step enzymatic pathway; each step requires one mole of ATP per substrate. The first step is regulated by MVA kinase as been demonstrated in plant 
species and microorganisms. The identity of MVA-5-pyrophosphate, the product resulting from the second step, has been evidenced in yeast and has been identified as an intermediate in a bacterial system that can convert MVA into carotenes. Isopentenyl pyrophosphate can be found in enzymatic systems capable of carotenoid synthesis, such as tomato fruit.

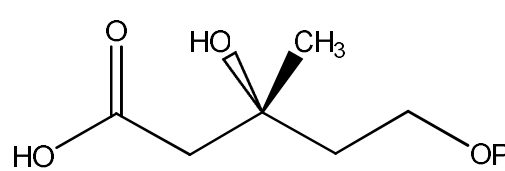

Mevalonate-5-phosphate

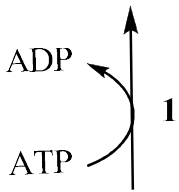

MVA
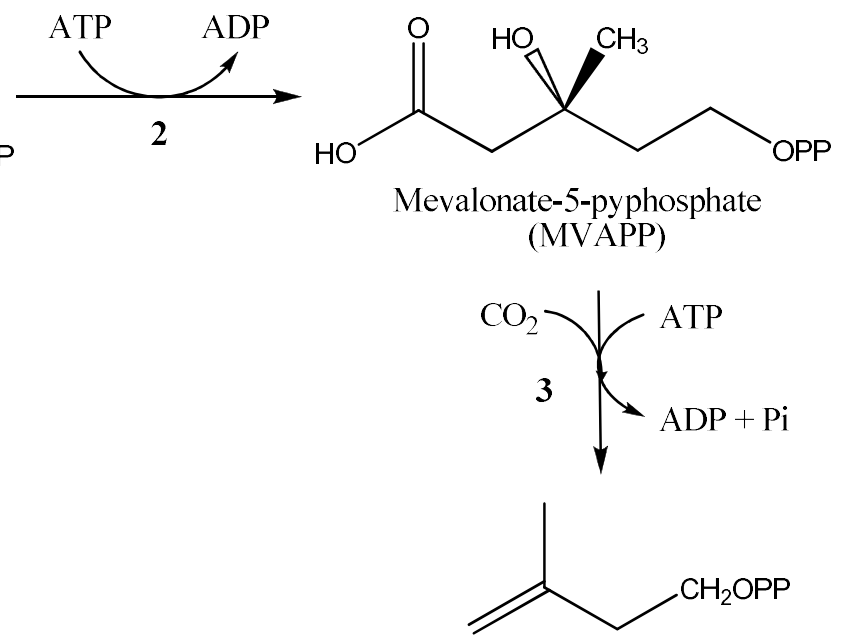

IPP

Figure 1. Formation of IPP from MVA

To continue chain elongation, IPP is isomerized to dimethyl lallyl diphosphate (DMAPP) in the first step by the enzyme IPP isomerase [8] (See Figure 1). The sequential addition of three IPP molecules to DMAPP, lengthens the chain to 10 carbons. Formation of the $\mathrm{C}_{15}$ molecular intermediate (FPP) is catalyzed by the enzyme geranylgeranyl diphosphate synthase (GGPS) that links DMAPP. The enzyme is one member of a family of closely related prenyl transferase enzymes distinguished by the 
length of the final product. The 20-carbon molecule geranyl geranyl pyrophosphate (GGPP) is generated by two identical $\mathrm{C}_{5}$ addition steps.

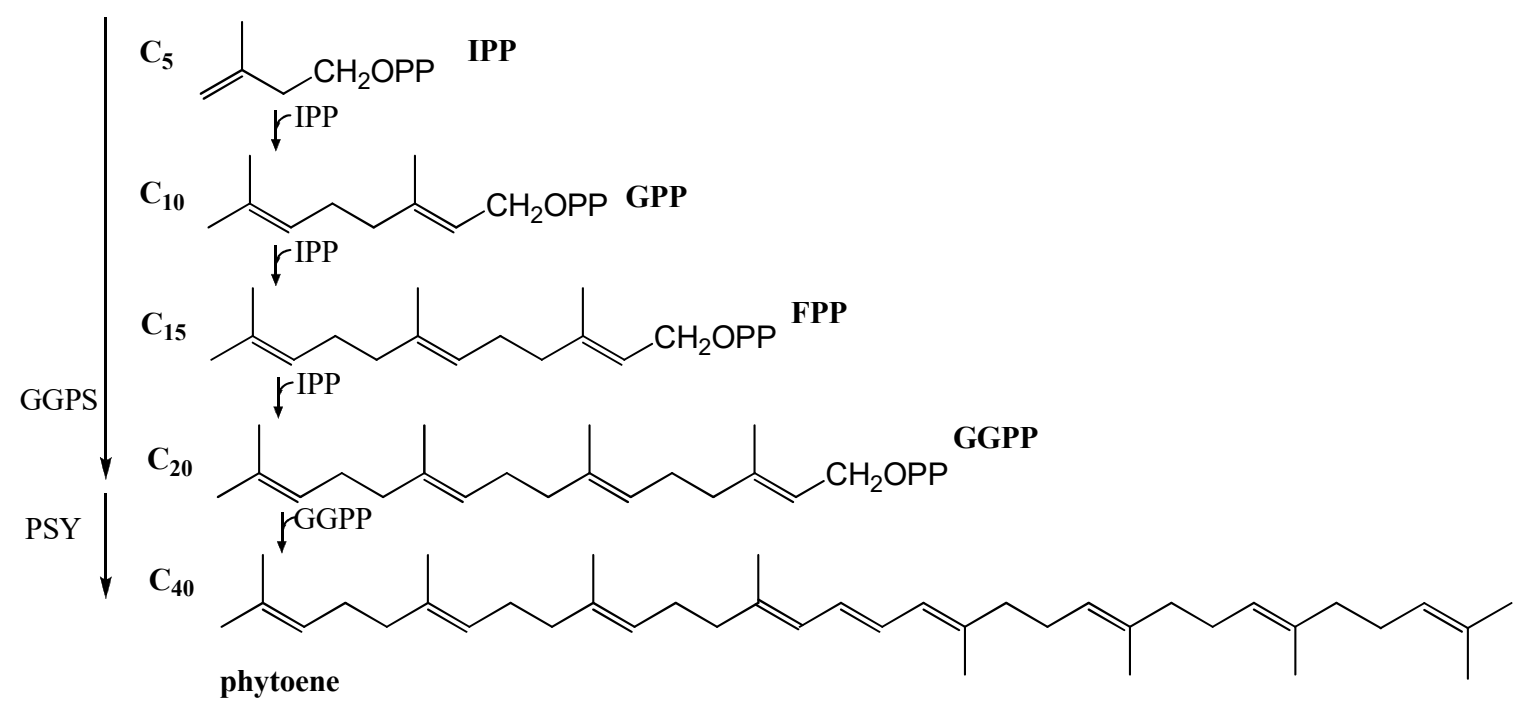

Figure 2. General pathway for the formation of phytoene [7]. The arrows on the side represent the enzymes involved. GGPS: geranylgeranyl diphosphate synthase. PSY: phytoene synthase.

The next step in the carotenoid pathway is the condensation of two GGPP molecules to produce phytoene, which is catalyzed by a membrane-associated enzyme, phytoene synthase (PSY). Phytoene synthase shares amino-acid sequence similarity with GGPP synthase and other prenyl-transferases [8]. The condensation of two molecules of farnesyl pyrophosphate to produce squalene, the $\mathrm{C}_{30}$ precursor of sterols, is similar to the formation of phytoene from geranylgeranyl diphosphate synthase. In the biosynthesis pathway, NADPH is not required and one hydrogen is lost from $\mathrm{C} 1$ of each of the participating GGPP molecules. The GG residues are linked via sulphonium ylide. 
Four desaturation steps are required for the conversion of phytoene to lycopene and are catalyzed by two related enzymes in plants: phytoene desaturase and $\zeta$-carotene desaturase. However, bacteria and fungi achieve the same result via a single enzymatic complex. The first desaturation of phytoene produces phytofluene; then phytofluene undergoes dehydrogenation and isomeration to be converted into $\zeta$-carotene. $\zeta$-carotene is subsequently desaturated to produce neurosporene and lycopene. In the end, the four reactions build the conjugated carbon backbone of lycopene.

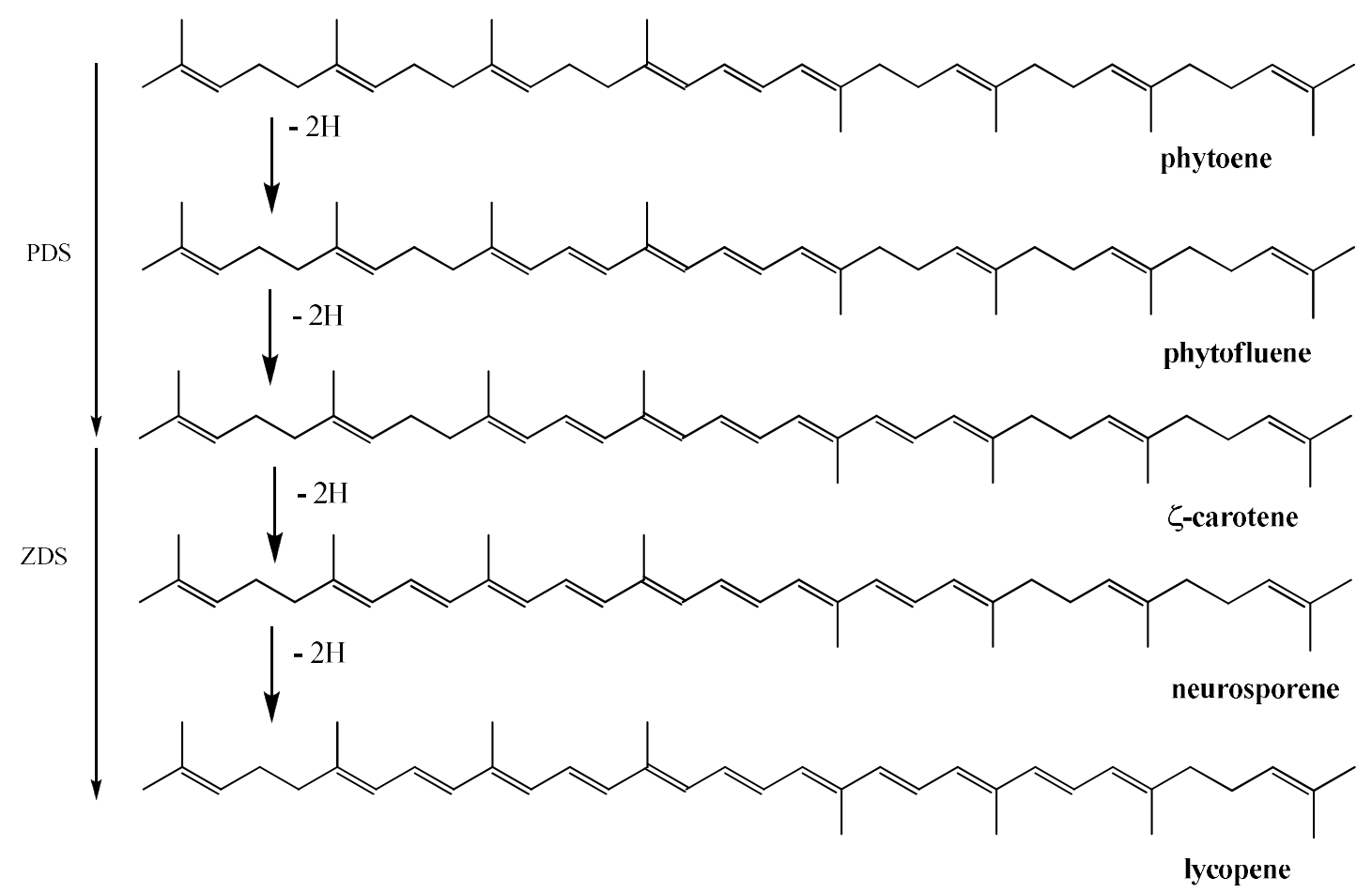

Figure 3. General pathway for the formation of lycopene [7]. The arrows on the side represent the enzymes involved. PDS: phytoene desaturase. ZDS: $\zeta$-carotene desaturase.

At this point in the biosynthesis, the pathway branches and the cyclization of lycopene may occur to produce either $\beta$ - or $\varepsilon$-ring end-group structures. One branch leads 
to the formation of $\beta$-carotene and its derivative xanthophylls, and the other branch leads to $\alpha$-carotene and lutein. $\varepsilon$-carotene is also produced from $\delta$-carotene.

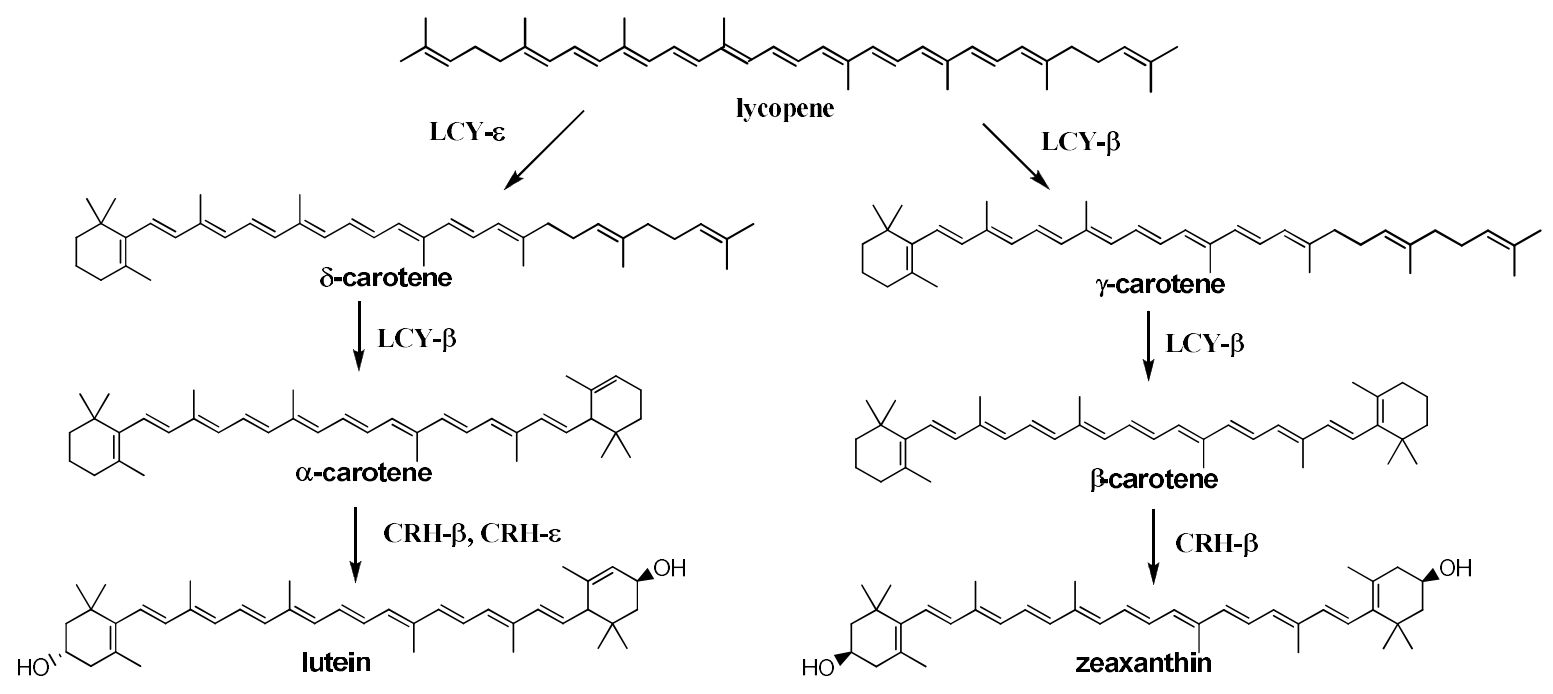

Figure 4. Cyclization of lycopene. Enzymatic conversions are shown by arrows with the enzymes responsible. LCY- $\beta$ : lycopene beta-cyclase; LCY- $\varepsilon$, lycopene epsilon-cyclase; $\mathrm{CRH}-\beta, \beta$-carotene hydroxylase; $\mathrm{CRH}-\varepsilon$, epsilon-carotene hydroxylase [8].

During the biosynthesis of the cyclic carotenoids, the formation of $\varepsilon$ - and $\beta$ ionone rings take place at a late stage in the pathway. Experiments indicate that the $\beta$ ionone ring is formed by a mechanism that involves a carbonium ion (Figure 5). The cyclization concludes with the release of the $\mathrm{H}_{\mathrm{B}}$ proton from the $\mathrm{C}-6$-ionone is also formed by via a carbonium ion but in this case it is stabilized by expulsion of the $\mathrm{H}_{\mathrm{A}}$ proton at $\mathrm{C}-4$.

During the cyclization of lycopene, the reactions that are necessary for the formation of the carotenoid end-groups in both branches (the one that leads to lutein and the other that leads to zeaxanthin, Figure 4) are dependent on the relative activities of the 
cyclases involved in the reaction. The most common xanthophylls in the green tissue of higher plants, algae, and non-photosynthetic bacteria have hydroxyl substitutents at C-3, an oxo at C-4 or an epoxy at the 5, 6 position of the ionone ring [9]. Genetic evidence and functional analysis of an Arabidopsis $\beta$-hydroxylase enzyme support the existence of separate hydroxylases specific for the $\beta$ - and $\varepsilon$-rings.

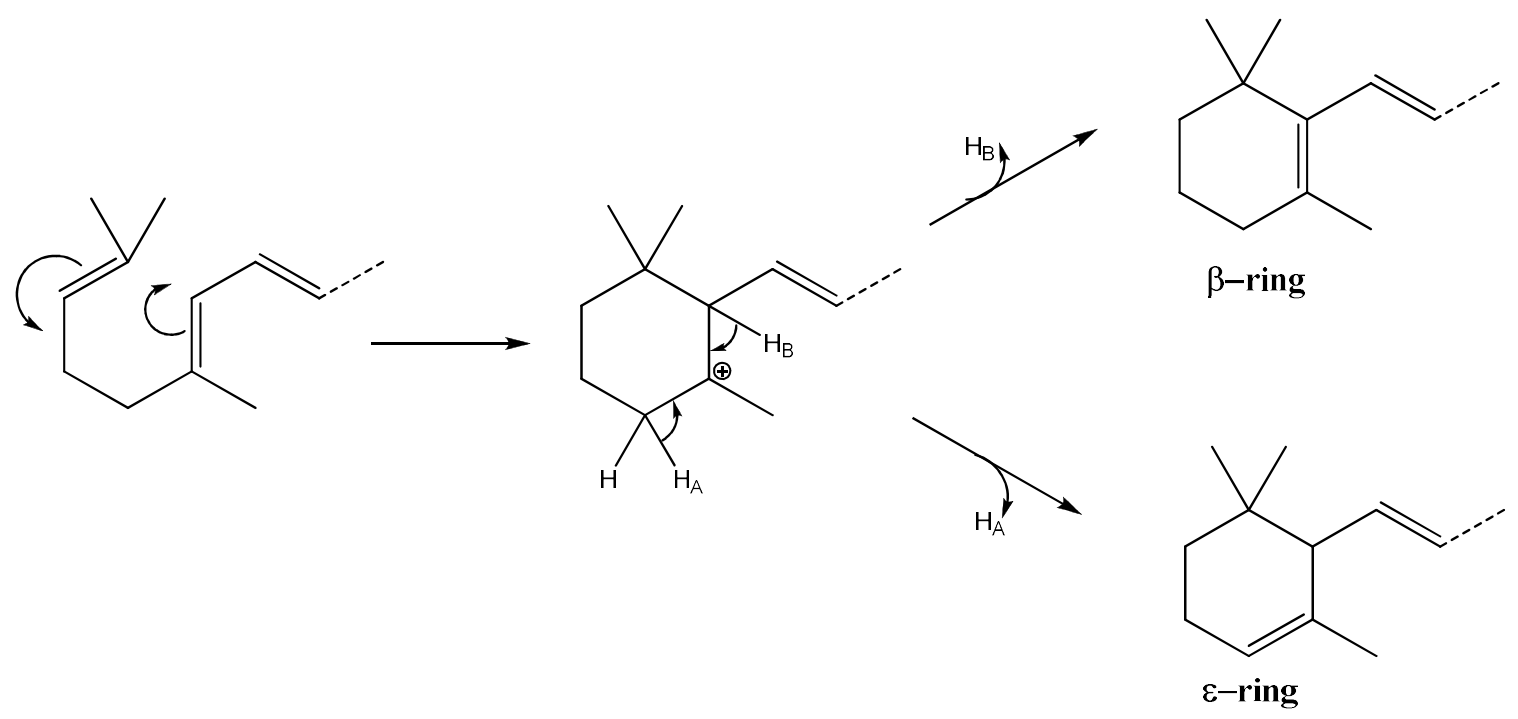

Figure 5. Basic mechanism for biosynthesis of $\varepsilon$ and $\beta$-rings in carotenoids.

\subsection{Carotenoid structures}

Carotenoids are tetraterpenoids that contain eight isoprenoid units [6], Figure 6. They have a $\mathrm{C}_{40}$ carbon skeleton. Isoprenoid units link together in a head to tail pattern to form two $\mathrm{C}_{20}$ fragments that are again linked together in a head to head fashion at the center to form a carotenoid unit. Therefore, neighboring methyl groups of the polyene chain are six carbons apart from each other at the chain center but only 5 carbons apart in 
rest of the molecule. As shown in the previous section, all $\mathrm{C}_{40}$ carotenoids are derived from lycopene by synthetic pathways that include: cyclization, oxygen insertion, double bond migration, methyl migration, chain elongation, and chain shortening [6].

A

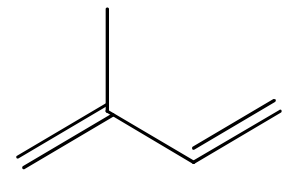

B

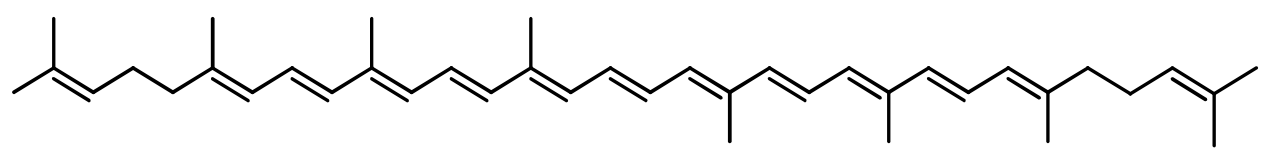

Figure 6. Carotenoid structure. A) Isoprene unit. B) C40 Structure of Lycopene.

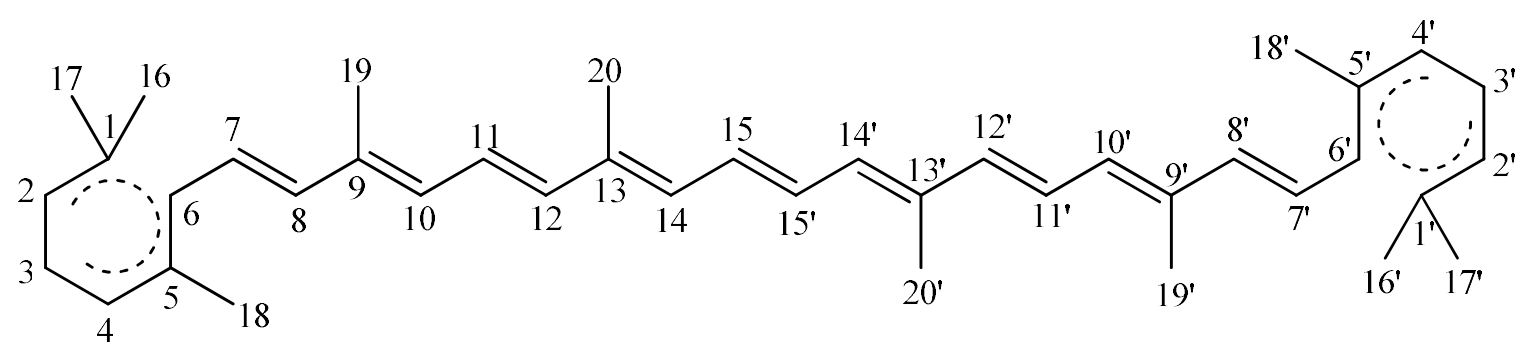

Figure 7. Carotene numbering system.

Traditionally, carotenoids can be grouped into two classes: (a) carotenes or (b) xanthophylls. Carotenes are hydrocarbons (example: lycopene or $\beta$-carotene). Xanthophylls have oxygen atoms in their structure (example: lutein and zeaxanthin) [10]. For carotenes, all specific names are based on the stem name "carotene", which corresponds to the structure and numbering shown in Figure 7. 

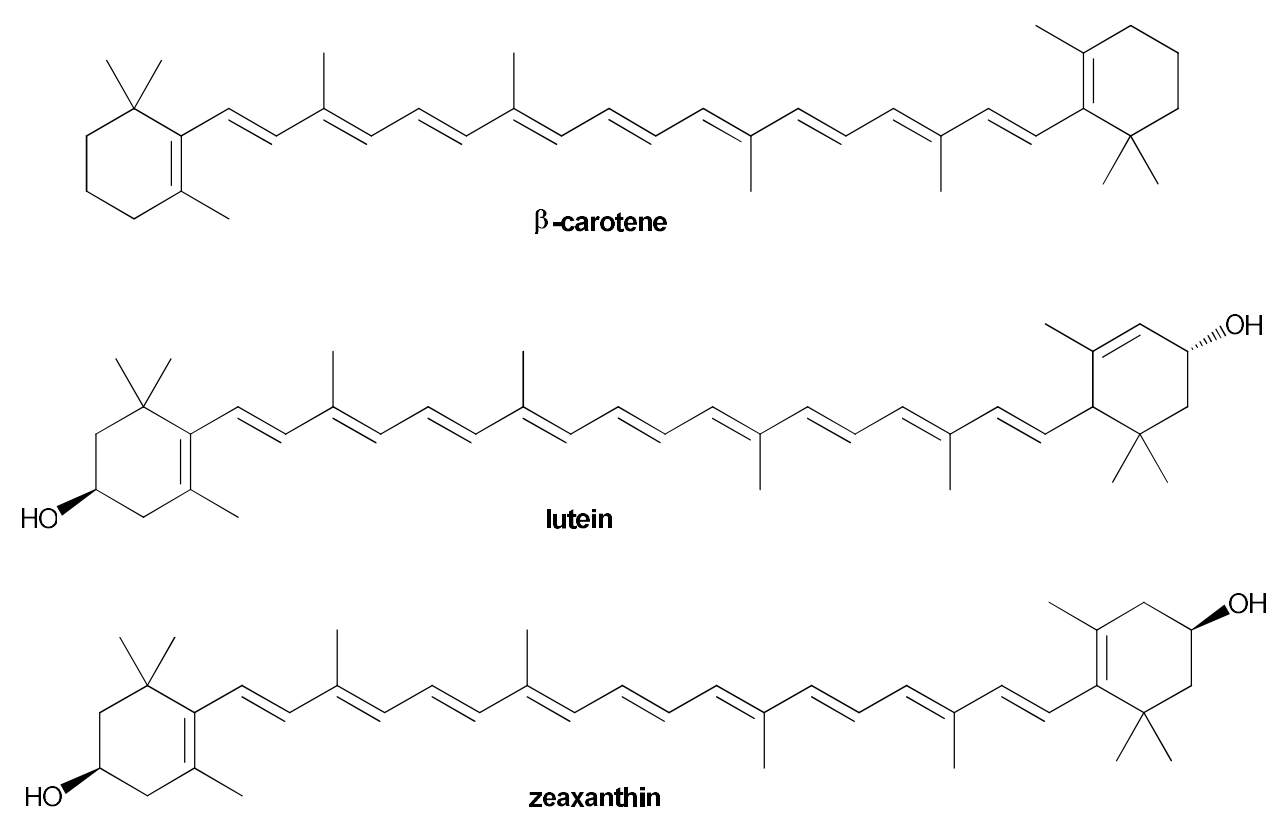

Figure 8. Carotene ( $\beta$-carotene) and Xanthophylls (lutein and zeaxanthin).

Table 1. End-Group Designations of Carotenes.

\begin{tabular}{|l|c|c|}
\hline \multicolumn{1}{|c|}{ Type } & Prefix & Structure \\
\hline Acyclic & $\psi$ & (a) \\
\hline Cyclohexene & $\beta, \varepsilon$ & $(\mathrm{b}, \mathrm{c})$ \\
\hline Methylenecyclohexane & $\delta$ & (d) \\
\hline Cyclopentene & $\varepsilon$ & $(\mathrm{e})$ \\
\hline Aryl & $\phi, \chi$ & $(\mathrm{f}, \mathrm{g})$ \\
\hline
\end{tabular}

Structurally, the carotenoids possess end-groups that are either acyclic or rings of five or six carbons at one or both ends of the molecule. Carotenes are named according to the end group(s) that are present in their structures[6]. The end-groups and their prefixes are indicated in Table 1.

In green tissues, algae, and many fungi the major carotenoids are cyclic compounds such as: $\beta$-carotene and $\alpha$-carotene. The carotenes mentioned contain ionone 
rings in their structure. $\beta$-carotene has two $\beta$-ionone groups and $\alpha$-carotene has one $\varepsilon$ ionone and one $\beta$-ionone ring.<smiles>[R]C=C(C)CCC=C(C)C</smiles>

(a) $\Psi$<smiles>[R]C1C(=C)CCCC1(C)C</smiles>

(d) $\gamma$

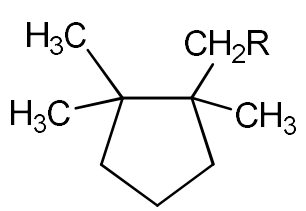

(e) $\kappa$<smiles>[R]C1=C(C)CCCC1(C)C</smiles>

(b) $\beta$<smiles>[R]c1c(C)ccc(C)c1C</smiles>

(f) $\phi$<smiles>[R]C1C(C)=CCCC1(C)C</smiles>

(c) $\varepsilon$<smiles>[R]c1ccc(C)c(C)c1C</smiles>

(g) $\chi$

Figure 9. End-groups structure

In xanthophylls, which contain oxygen functional groups, the compounds are named according to the usual IUPAC nomenclature rules, indicating the position of the oxygen containing substituent. For example, the name of zeaxanthin (see Figure 8 ) is $\beta$, $\beta$ carotene-3,3'-diol.

The conjugated double bonds present in the structures of carotenoids give them the possibility to exist as several "cis-trans" geometrical isomers. Usually, cis and trans are used to indicate the orientation of substituent groups in a molecule relative to a double bond. When there are more than two different substituents on a double bond, $E$ and $Z$ are the more correct designations used to describe the geometrical isomerism. In 
nature, most carotenoids are found in the all-E (trans) configuration. It is also possible for the structures to have one or more "cis" or $Z$ double bonds, but these are energetically disfavored.

\subsection{Physical and Chemical properties}

The size and shape of the carotenoid molecule are important in determining its properties and functionality. All carotenoids in the all-trans configuration have an extended conjugated double-bond system, in cells they are soluble in the hydrophobic components and especially the interior of the cell membrane [11]. Polar xanthophylls are known to span the cell membrane, placing the polar hydroxyl groups at the membrane surface; whereas more non-polar carotenoids, such as $\beta$-carotene take a position fully embedded within the hydrophobic interior [12]. The shape of cis carotenoid isomers differs from that of the all-trans forms, and consequently their fit into subcellular structures is altered. Carotenoids may be associated with other molecules in the living cell such as proteins and lipids, a result of their size, shape, and lipophilicity. Likewise, for some carotenoids, the presence of polar functional groups on their structure gives them the ability to interact with other polar molecules. The presence of carotenoids in the cell membrane affects the membrane's thickness, strength, fluidity, and permeability to water, oxygen, and other molecules. Interactions between carotenoids and proteins are common in living organisms [11]. They are associated with the lipid components of lipoproteins, which allows them to be transported and to function in aqueous

environments [11]. In vivo, carotenoids are more stable than when isolated in pure compounds, especially when in organic solution. 


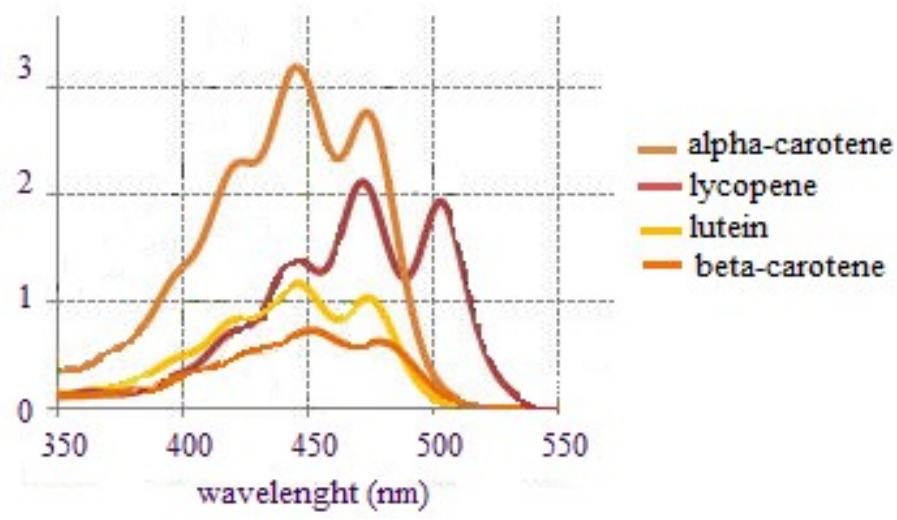

Figure 10. Electronic absorption spectra of different carotenoids (extracted from tomatoe in $80 \%$ acetone/diethyl ether). Taken from [13]

Most carotenoids are unstable and in the presence of light and oxygen are readily degraded. The ability of carotenoids to absorb light is conferred by the presence of the extended conjugation of double bonds present in the polyene chain. Most carotenoids absorb light in regions of the visible and ultraviolet spectrum where few other biological compounds absorb. Many carotenoids show three peaks or two peaks with a shoulder absorbance spectrum around $450 \mathrm{~nm}$ [13]. Their characteristic visible absorption spectrum is principally a consequence of the number of bonds in the conjugated chain but is, in addition, modified by other functional groups in the structure. A result of an additional conjugated double bonds is an increase of between $7-35 \mathrm{~nm}$ in the wavelength maximum. [14]. Geometrical isomerization also influences the electronic absorption spectra of carotenoids.

\subsection{Antioxidant activity of carotenoids $[15,16]$}

Carotenoids have an important role protecting cells and organisms against damage by light and reactive oxygen species (ROS) generated by light [17] and allow them to act 
as antioxidants. Because of their ability to accept energy through space from neighboring excited state species via a quantum coupling mechanism or by collisional interactions and to rapidly relax dissipating energy through vibrionic interconversion, carotenoids are very effective at quenching and inactivating those high-energy excited states resulting from the absorption of short wavelength light by molecules that are capable of activating oxygen, particularly hemes, chorophyll, and vitamin A. Krinsky elegantly explained how carotenoids perform as antioxidants by reacting with reactive singlet oxygen generated during photosynthesis and in other biological systems exposed to both light and oxygen $[16]$.

1.4.1 Carotenoid quenching of singlet molecular oxygen $\left({ }^{1} \mathrm{O}_{2}\right)$

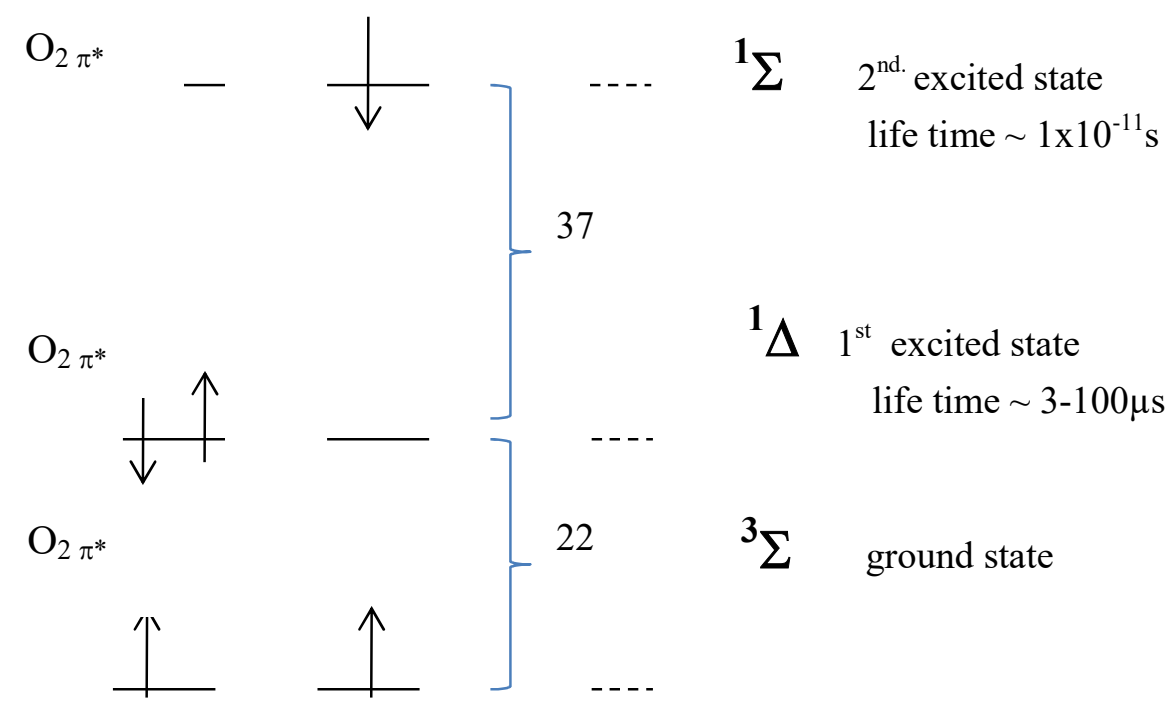

Figure 11. Diagram of the electronic states of the $\mathrm{O}_{2}$ molecule.

When light of the correct energy strikes a biological system that contains an appropriate sensitizer (S), the energy of the photon is absorbed and the sensitizer is electronically excited, most commonly to produce the ${ }^{1} \mathrm{~S}$, excited state. The singlet 
excited states for most molecular species are very short-lived. The lifetime, $\tau$, for most species is measured in nanoseconds (typically $\tau \sim 0.1-100 \times 10^{-9} \mathrm{~s}$ ). By intersystemcrossing the singlet excited states, ${ }^{1} \mathrm{~S}$, can efficiently interconvert to a longer lived triplet excited state, ${ }^{3} \mathrm{~S}$ (Equation 1).

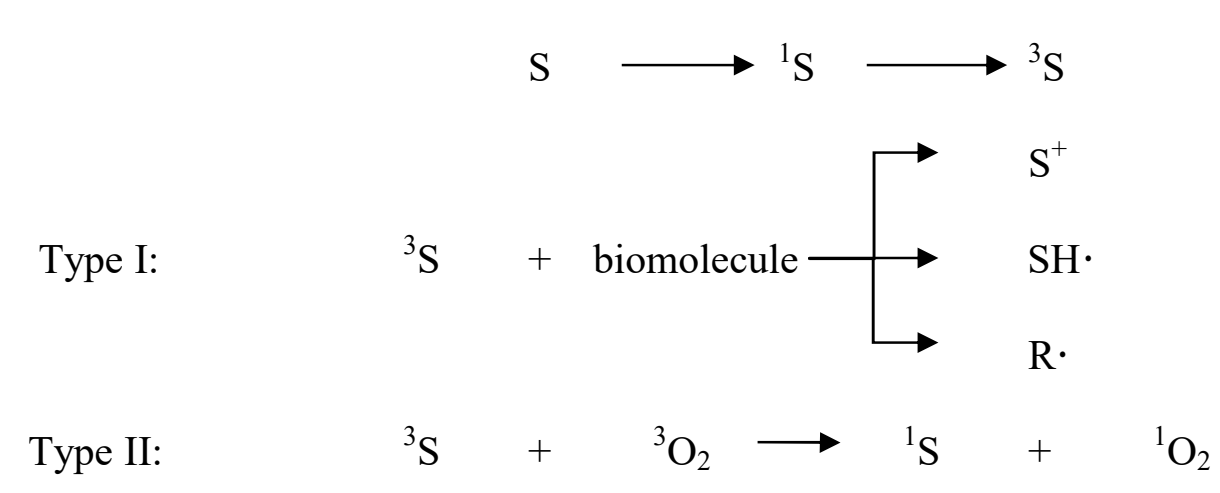

The ${ }^{3} \mathrm{~S}$ can initiate Norrish Type I or Type II photochemical reactions. In Norrish Type I reactions the triplet initiates radical reactions by abstracting an electron or hydrogen atom from nearby biomolecules creating reactive free radical species, Equation 2. In the Norrish Type II reaction, the triplet sensitizer reacts with molecular oxygen. $\mathrm{O}_{2}$, also a triplet species, to form of the highly reactive ${ }^{1} \mathrm{O}_{2}$, regenerating the ground-state sensitizer, Equation 3, while conserving the total spin of the system as required by quantum mechanics.

Ground state carotenoid interacts readily with ${ }^{1} \mathrm{O}_{2}$, accepting the excess energy and quenching this reactive excited state, Equation 4. This reaction also conforms to the quantum mechanical requirement that net spin be conserved during any allowed process.

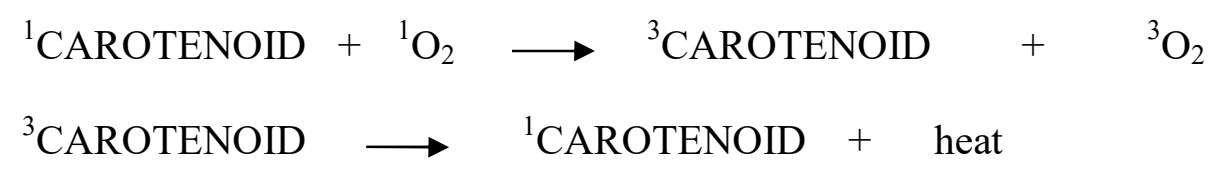


The geometry of the linear conjugated polyene chain confers on the carotenoid molecules a large number of closely spaced vibrational energy states that facilitate the dissipation of energy from the excited state through vibrational and rotational transitions. Coupled with the process of energy transfer involve in intermolecular collisions with the solvent energy is readily and harmlessly lost from the excited state carotenoid to the surrounding as excess heat. Thus the triplet carotenoids return to their ground state unaltered and ready to repeat the process of quenching, Equation 5.

Lipophilic carotenoids rapidly react with peroxyl radicals competing for free radicals generated when free radicals react with oxygen. Peroxyl radicals are formed during lipid peroxidation and scavenging of this species interrupts the chain reaction sequence that can rapidly produce extensive damage to unsaturated species in lipophilic compartments [18]. Therefore, carotenoids play an important role in the protection of cellular membranes and lipoproteins against oxidative damage because of their lipophilicity and ability to scavenge peroxyl radicals [19].

\subsection{Bioactivity and disease prevention}

In humans, carotenoids act as antioxidants by neutralizing the ROS in cellular tissues, as described above. ROS are generated by normal metabolic activity as well as photic processes and many environmental and behavioral factors may contribute to their formation. These include smoking, air pollutants, diet, and exposure to either natural or industrial chemicals [20]. Studies have demonstrated that carotenoids are positively correlated with a reduced risk of many diseases that are associated with oxidative 
processes, including some types of cancer, cardiovascular disease, and age-related macular degeneration [21], (see Table 2). Carotenoids may also act to optimize health through mechanisms such as promoting transcription of gap junction proteins and associated gap junction communication, effects on regulation of cell growth, modulation of gene expression and immune response, and as modulators of Phase I and II drug metabolizing enzymes [22].

In other in vitro studies, $\beta$-cryptoxanthin has been found to have a stimulatory effect on bone calcification and, osteoblastic bone formation, and inhibitory effects on osteoclastic bone resorption. Moreover, in animal models the intake of $\beta$-cryptoxanthin appears to have a preventive effect on bone loss leading to osteoporosis and may also function in this manner in healthy humans or postmenopausal women [23].

\subsection{Transport and distribution of carotenoids in the human body}

In animal models, the pathway for absorption and metabolism of carotenoids starts after the consumption of carotenoids from the daily diet [24]. As mentioned above, carotenoids are implicated in the prevention of degenerative diseases. They have a lower bioavailability than other lipophilic components such as $\alpha$-tocopherol and may be a factor that influences their action. The main cause of this lowered bioavailability is the poor solubility of carotenoids in digestive fluids [25]. 
Table 2. Carotenoids, functions and effects associated with prevention of human diseases and optimization of human health (taken from Takana et al.[26]).

\begin{tabular}{|c|c|c|c|}
\hline Carotenoids & Dietary Sources & Function & Effects \\
\hline$\alpha$-Carotene & $\begin{array}{l}\text { Yellow-orange vegetables } \\
\text { (carrots, sweet totatoes, } \\
\text { pumplin) and Dark-green } \\
\text { vegetables (broccoli, green } \\
\text { beans, spinach) }\end{array}$ & $\begin{array}{l}\text { Provitamin A activity; } \\
\text { Anti-oxidant }\end{array}$ & $\begin{array}{l}\text { Immune- enhancement; Stimulate } \\
\text { cell to cell communication; } \\
\text { Decreases risk of some cancers }\end{array}$ \\
\hline$\beta$-Carotene & $\begin{array}{l}\text { Green leafy vegetables and } \\
\text { orange and yellow fruits and } \\
\text { vegetables (carrots, apricots, } \\
\text { spinach, sweet potetoes, } \\
\text { pumplin, pepper, kale, } \\
\text { cantaloupe) }\end{array}$ & $\begin{array}{l}\text { Provitamin A activity; } \\
\text { Antioxidant }\end{array}$ & $\begin{array}{l}\text { Immune-enhancement; Decreases } \\
\text { risk of some cancers and some } \\
\text { cardiovascular events; high-dose } \\
\text { supplementation may increase the } \\
\text { risk of lung cancer among smokers }\end{array}$ \\
\hline Lycopene & $\begin{array}{l}\text { Tomatoes, water melon, } \\
\text { apricot, peaches }\end{array}$ & Anti-oxidant & $\begin{array}{l}\text { Decreases risk of some cancers } \\
\text { and some cardiovascular events, } \\
\text { diabetes, and osteoporosis }\end{array}$ \\
\hline$\beta$-Cyptoxanthin & $\begin{array}{l}\text { Orange fruits (mandarin } \\
\text { orange and papaya, etc.), } \\
\text { corn, peas, and egg yolks }\end{array}$ & $\begin{array}{l}\text { Provitamin A activity; } \\
\text { Anti-oxidant }\end{array}$ & $\begin{array}{l}\text { Anti-inflammatory effects; Inhibits } \\
\text { risks of some cancer and } \\
\text { cardiovascular events; Immune } \\
\text { enhancement }\end{array}$ \\
\hline Lutein/Zeaxanthin & $\begin{array}{l}\text { Dark green leafy vegetables } \\
\text { (spinach, kale), red peppers, } \\
\text { maize, tomatoes, corn, and } \\
\text { egg yolks }\end{array}$ & $\begin{array}{l}\text { Anti-photosensitizing } \\
\text { agent and photosynthetic } \\
\text { pigment; Acts as } \\
\text { antioxidants and blue } \\
\text { light filters }\end{array}$ & $\begin{array}{l}\text { Decrease age-related macular } \\
\text { degeneration, cataract, and risk of } \\
\text { cardiovascular disease and certain } \\
\text { cancers }\end{array}$ \\
\hline Astaxanthin & $\begin{array}{l}\text { Green algae, salmon, trout, } \\
\text { crustacea }\end{array}$ & Antioxidant; Coloration & $\begin{array}{l}\text { Prevent certain cancers, cataract, } \\
\text { diabetes, and inflammatory } \\
\text { neurodegenerative and } \\
\text { cardiovascular diseases }\end{array}$ \\
\hline Canthaxanthin & Salmon, crustacea & Antioxidant; Coloration & $\begin{array}{l}\text { Immune enhancement; Decreases } \\
\text { risk of some cancers }\end{array}$ \\
\hline Focoxanthin & Brown algae, heterokonts & Antioxidant & $\begin{array}{l}\text { Anti-cancer, anti-allergic, } \\
\text { anti-obese, anti-inflammatory, and } \\
\text { anti-osteoporotic activities }\end{array}$ \\
\hline
\end{tabular}

Carotenoids are lipid-soluble and follow absorption pathways similar to those of other dietary lipids, but the pathway is more complex because there are several additional steps. We should mention here that digestion and absorption of dietary lipids is a prerequisite for optimum absorption of carotenoids in the body [26]. Carotenoids are incorporated into a complex food matrix that contains proteins and lipids. After the food 
matrix is digested, carotenoids are combined with lipids and bile salts in micelles. The polarity of each individual carotenoid influences the extent to which it is incorporated into micelles. [27]. The micelles, together with incorporated carotenoids, are captured and transported into the enterocyte at the intestinal brush border membrane. Once in the enterocyte, micelles are deconstructed and components may be metabolized. Carotenoids appear to be absorbed by the mucosa of the small intestine (mainly in the duodenum) via passive diffusion to become packaged into triacylglycerol-rich chylomicrons. Carotenoids repackaged in chylomicrons are secreted into the hepatic portal vein leading to their transport to the liver for further reprocessing. Sugawara et al.[28] investigated the absorption of 15 different carotenoids solubilized in mixed micelles in differentiated Caco-2 human intestinal cells, as a model of absorption by intestinal cells and showed a linear correlation between carotenoid uptake and lipophilicity (defined as their partition coefficient in 1-octanol/water). Therefore, the uptake of the relatively lipophilic carotenes by Caco-2 cells is higher than that of the less lipophilic xanthophylls. Carotenoids that are more lipophilic can diffuse more easily through the membrane than the less lipophilic ones. Provitamin A carotenoids, such as $\alpha$-, and $\beta$-carotene and $\beta$ cryptoxanthin, are partly converted to vitamin A (primarily retinyl esters) in the intestinal mucosa cells. Carotenoids and retinyl esters are incorporated into chylomicrons and secreted into lymph for delivery to the blood stream, where the chylomicrons are rapidly degraded by lipoprotein lipase [27]. The remaining chylomicron containing carotenoids are taken up by the liver, where they can be processed and secreted as a component of hepatic VLDL (very low-low density-lipoprotein), LDL (low density lipoproteins) and 
HDL (high density lipoproteins). Carotenoids that are not filtered from circulation in the liver can be captured by extra-hepatic tissues before they are taken to the liver.

\subsection{Pro-vitamin A and non-pro-vitamin A carotenoids}

1.7.1. $\beta$-carotene, $\alpha$-carotene, and $\beta$-cryptoxanthin (pro-vitamin A carotenoids)

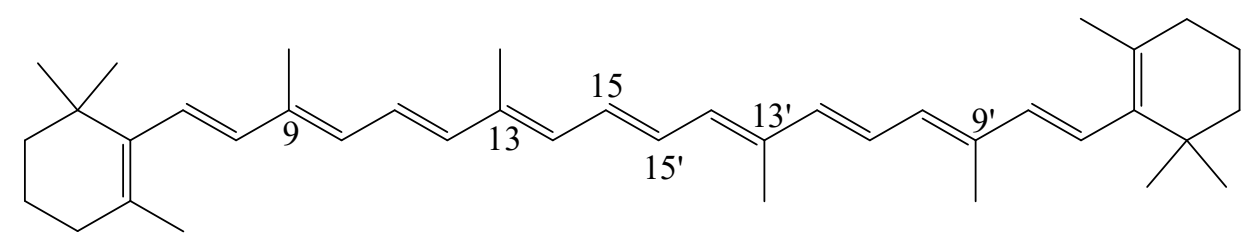

$\beta$-carotene

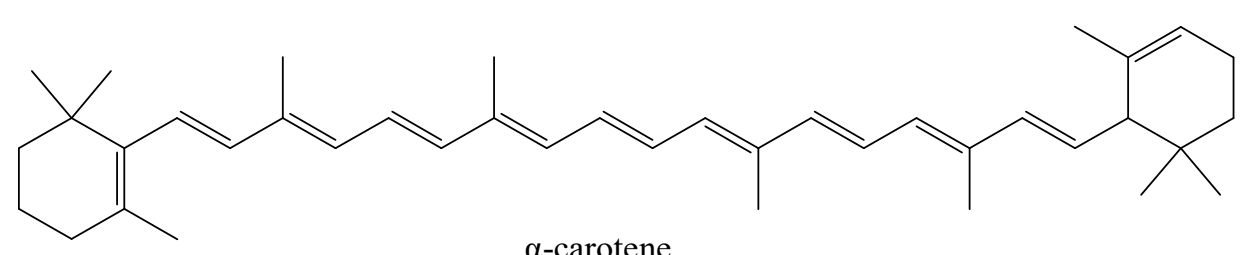

$\alpha$-carotene

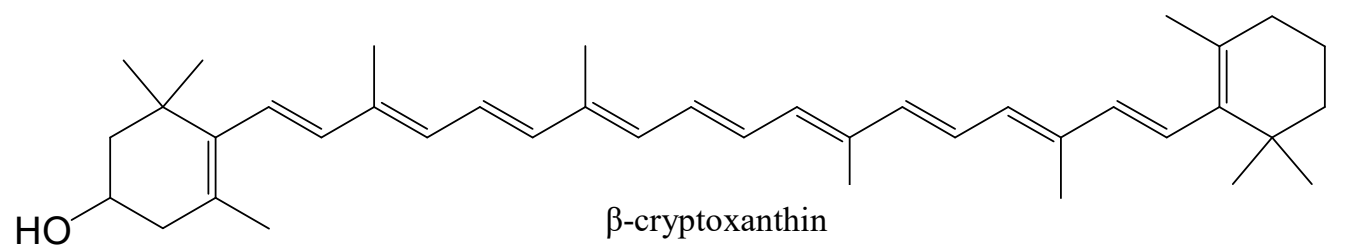

Figure 12. Examples of provitamin A carotenoids

In order to have pro-vitamin A activity, the carotenoid must have at least one unsubstituted $\beta$-ionone ring with methyl groups present in the correct numbers and positions on the polyene chain [29], see Figure 12. The conversion of one mole of $\beta$ carotene (two $\beta$-ionone rings in its structure, see Figure 12) theoretically gives two moles of retinal, while the conversion of one mole of $\beta$-cryptoxanthin, $\alpha$-carotene, or $\gamma$-carotene (each one with one $\beta$-ionone ring) gives only one mole of retinal $[29,30]$. Retinal is a 
direct precursor of retinol (vitamin A) and retinoic acid. The conversion efficiency in vivo is much lower and the exact stoichiometry of the process is much debated.

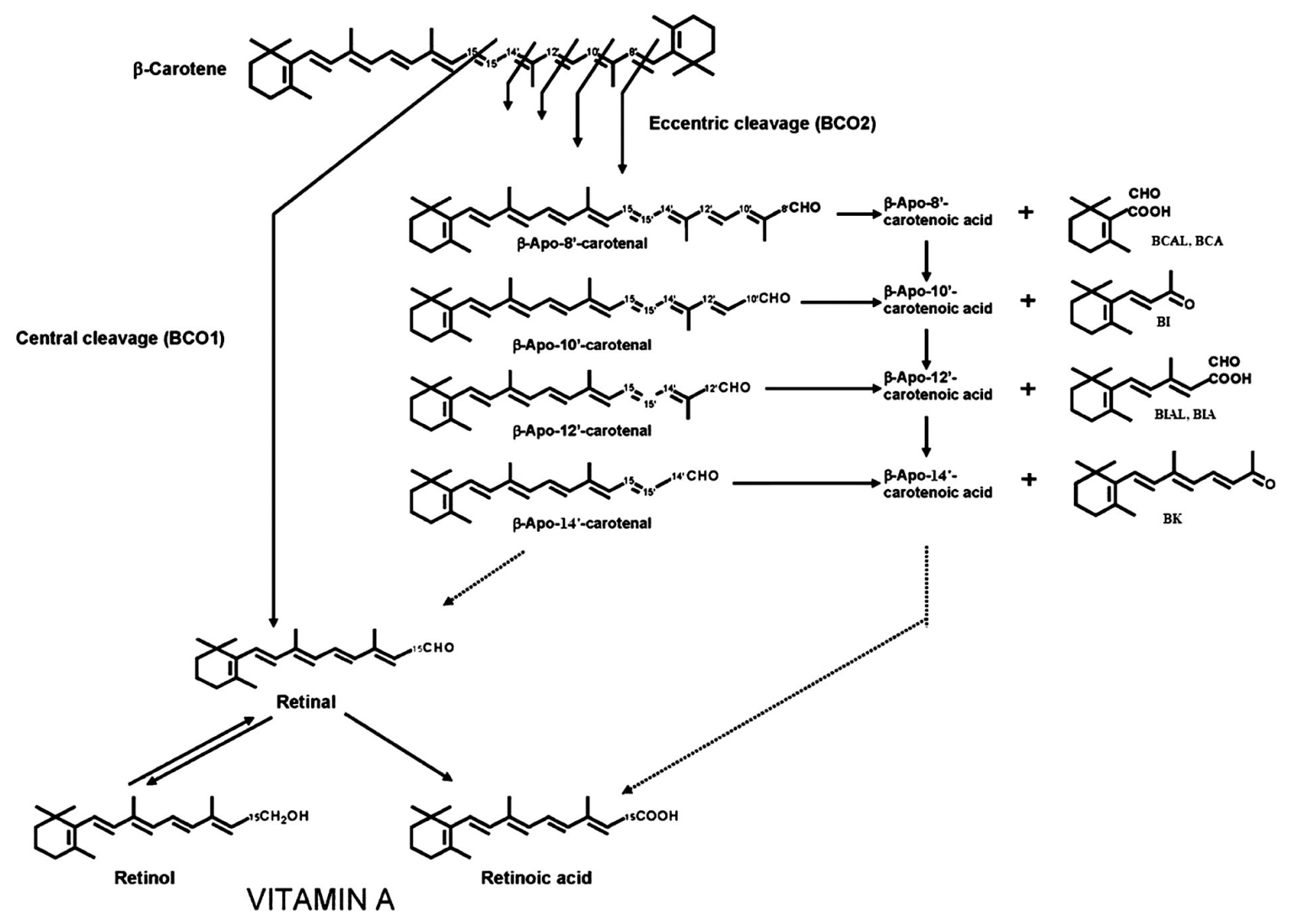

Figure 13. Central and eccentric cleavage of $\beta$-carotene (taken from Harrison [32]).

$\beta$-Cryptoxanthin is most prevalent in the diet as esters in fruits and vegetables. However these esters must be hydrolyzed to become bioavailable [30]. $\beta$-carotene is the most potent vitamin A precursor of all carotenoids; while $\alpha$-carotene, $\beta$-cryptoxanthin, and $\gamma$-carotene show 30 to 50\% of pro-vitamin A activity and 9-cis and 13-cis isomers of $\beta$-carotene less than $10 \%$, compared to all-trans $\beta$-carotene [29]. The highest concentrations of vitamin A as pro-vitamin A carotenoids are found in carrots, sweet potatoes, pumpkin, kale, spinach, collards and squash, roughly 5-10 $\mathrm{mg}$ retinol activity 
equivalents per $100 \mathrm{~g}$ (a retinol activity equivalent is equal to $1 \mu \mathrm{g}$ retinol or $12 \mu \mathrm{g} \beta$ carotene, or $24 \mu \mathrm{g}$ of $\alpha$-carotene or $\beta$-cryptoxanthin) [31].

The conversion of pro-vitamin A carotenoids into vitamin A (retinoids) has been described to occur by two pathways: central and eccentric cleavage. Both pathways are presented in Figure 8. The principal pathway by which retinal is formed is that of central cleavage and $\beta$-carotene is converted to retinal by the cytosolic enzyme, $\beta$-carotene$15,15^{\prime}$-oxygenase 1 (BCO1). BCO1 cleaves $\beta$-carotene at its central double bond $\left(15,15^{\prime}\right)$ to produce retinal. For the enzymatic central cleavage two mechanisms have been proposed, one by a dioxygenase reaction and one by a monooxygenase reaction mechanism [29]. In the second pathway, eccentric cleavage occurs at double bonds other than the central $15,15^{\prime}$ double bond of the polyene chain. $\beta$-carotene can be cleaved to produce $\beta$-apo-carotenals having different chain lengths. The cleavage at the $9^{\prime}, 10^{\prime}$ double bond is catalyzed by $\beta$-carotene- $9^{\prime}, 10^{\prime}$-oxygenase $2(\mathrm{BCO} 2)$. The two major sites of $\beta$-carotene conversion to vitamin $\mathrm{A}$ in humans are the intestine and liver. In the intestine about half the dietary pro-vitamin A carotenoids are converted to retinol (presumably in the enterocyte, but possibly some conversion occurs in the lumen through the action of enzymes secreted in the bile) and about half are absorbed intact; however, the conversion varies between individuals [29]. 
1.7.2 Lutein and Zeaxanthin (non-pro-vitamin A carotenoids)

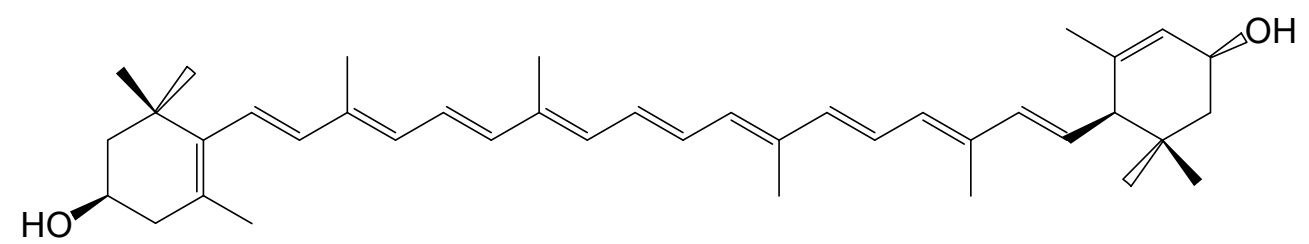

lutein

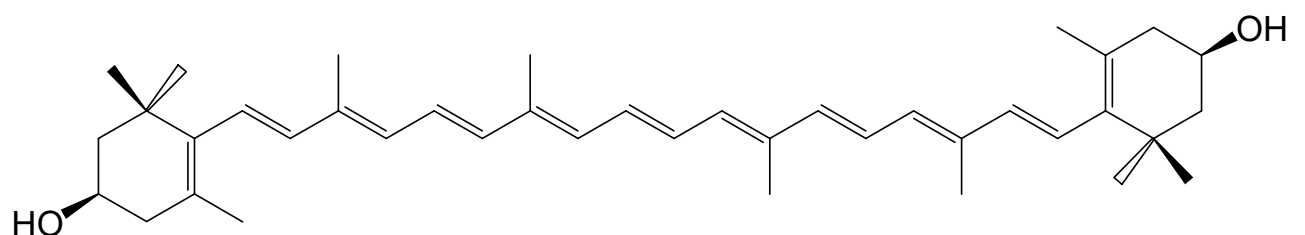

zeaxanthin

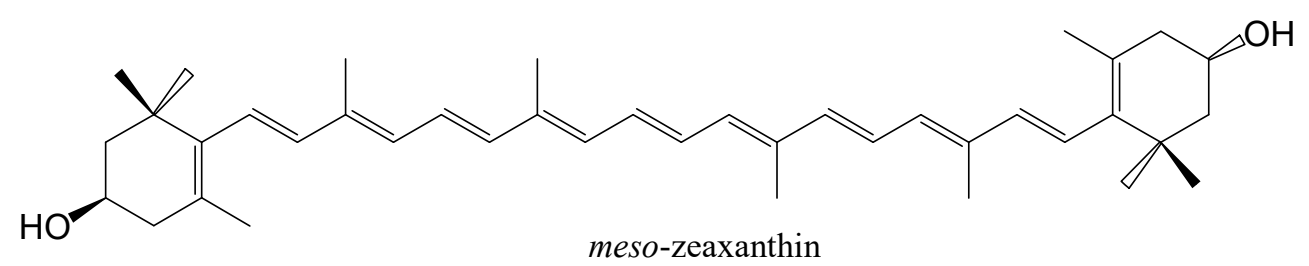

Figure 14. Major components of the macular pigment.

Like the other carotenoids, lutein and zeaxanthin are found abundantly in nature.

Humans obtain them only from their diet and they are most abundant in green leafy vegetables, fruits, and egg yolk. These two yellow carotenoids are selectively accumulated in the macular area of the retina. They are also known as macular pigment carotenoids.

The structural difference between lutein and zeaxanthin is found in a single endgroup (the $\varepsilon$-ring of lutein differs from the $\beta$-ring found in zeaxanthin). The configuration of the hydroxyl group in the $\beta$ - ring of zeaxanthin has a different stereo-chemical configuration than that in the $\varepsilon$-ring of lutein (see Figure 14). Dietary lutein is metabolized to produce meso-zeaxanthin within the retina, although the exact mechanism remains unproven [32]. 


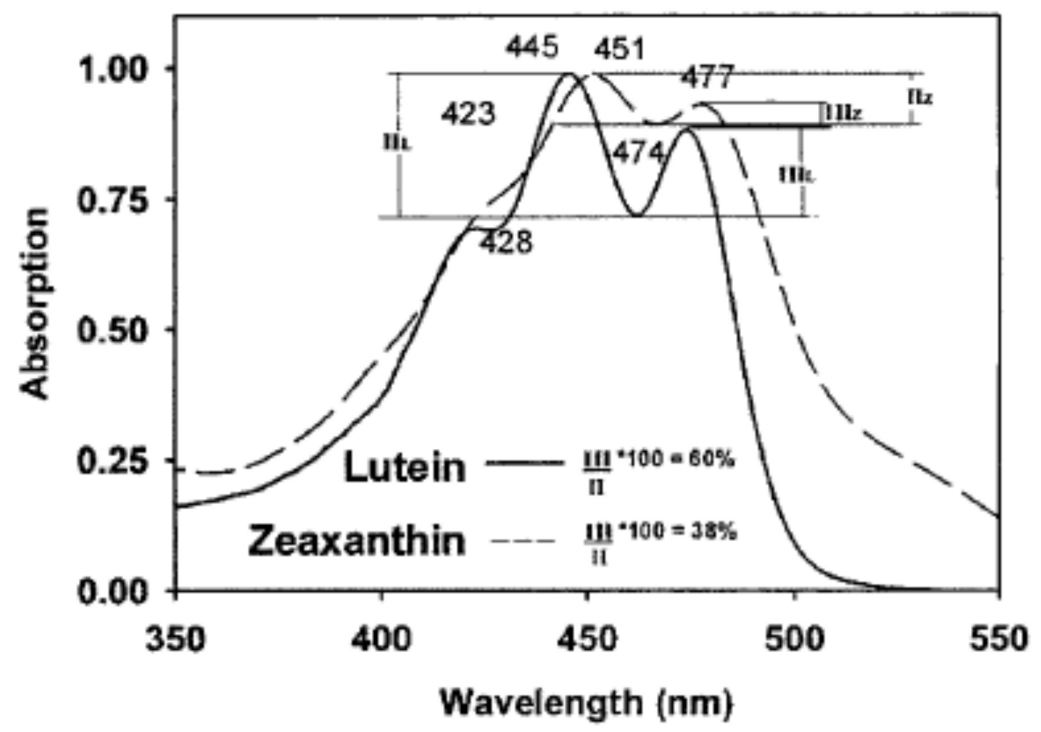

Figure 15. Spectra of lutein and zeaxanthin in ethanol[35].

Lutein and zeaxanthin are characterized by unique but similar UV-visible spectra (Figure 15). The presence of two $\beta$-ionone end-group rings in the zeaxanthin structure extends the conjugation of the polyene chain and lowers the energy separation between the ground and excited state, shifting its absorption spectrum slightly to the red. Lutein absorbs light of slightly shorter wavelengths than zeaxanthin, (see figure 14).

\section{A. Lutein and zeaxanthin in visual development}

Bone and Landrum showed that the major components of the macular pigment were lutein, zeaxanthin, and meso-zeaxanthin [32]. The macula is a yellow region with 5-6 $\mathrm{mm}$ of diameter (in adults) located in the posterior pole of the retina and it is unique to humans, see Figure 11. Lutein and zeaxanthin are concentrated in the fovea, a depression located in the central macula coinciding with the position of maximum visual acuity. These carotenoids have also been detected in the lens and the ciliary body [33]. The 
concentration of these carotenoids in the central retina approaches $1 \mathrm{mM}$, more than 10,000 times the concentration in human serum or the liver [34]. The major roles of lutein and zeaxanthin in the eye are believed to be: enhancement of visual acuity by decreasing glare and chromatic aberrations; and protection against light-induced oxidative damage by filtering blue light incident on the retina and by scavenging ROS in the retina tissue [35].

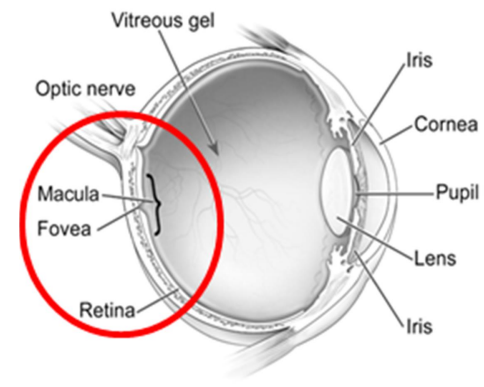

A

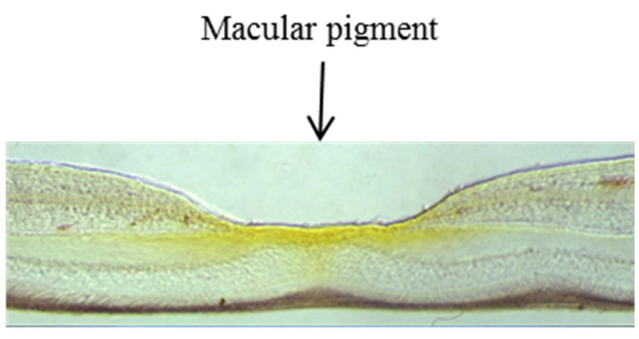

B

Figure16.A) Human retina, taken http://www.gene.com/gene/products/education/tgr/eye-health-glossary.html. B) Frozen section through the fovea of a rhesus monkey, taken from the Snodderly Lab, http://www.sbs.utexas.edu/SnodderlyLab/galler.

Studies have shown that lutein and zeaxanthin are present in prenatal eye as early as about 17 to 22 weeks of gestation, but there is no visible yellow spot (macula lutea) [36]. In the neonate human retina [37], lutein is the major carotenoid found in the central region, where the macula will develop, while in the fully developed retina zeaxanthin is the major carotenoid of the macula. The foveal depression in the infant macula does not develop until approximately 16 weeks of age, and foveal cones (which are located in the center of the fovea) do not become fully mature until approximately 4 years of age. Cones are responsible for color vision and for high spatial acuity. The transition to foveal 
zeaxanthin predominance occurs sometime before 3 years of age [38]. Studies in nonhuman primates by Leung et al. [39] indicate that lifetime deprivation of dietary lutein and zeaxanthin and a low intake of n-3 fatty acids lead to changes in the fovea and retina damage, suggesting that both macular pigment carotenoids and n-3 fatty acids are essential for development and/or maintenance of the central retina.

The retina is susceptible to oxidative stress because of the high concentrations of oxygen and the intensity of energetic short wavelength light. Infant retinas may be more susceptible to light damage than adults because of the greater transmission of higherenergy blue light through the infant's crystalline lens, which is not fully mature at birth $[40]$.

\section{B. Lutein in neuro development}

It has been reported that lutein is the predominant dietary carotenoid in human brain tissue in the infant $[41,42]$. Studies of primates show that concentration of lutein in the retina is significantly related to the concentration lutein in the brain tissue [43]. Also, in adults it was shown that cognitive functions are related to macular pigment density and lutein supplementation is correlated with improved cognitive performance in a small study of elder subjects [44]. Leibelin-Boff et al.[42] found a correlation between lutein concentration and different fatty acids in the infant brain tissue; in addition, they found a positive correlation between amino acid neurotransmitters and lutein. In other studies in mouse models, $[45,46]$ lutein was shown to have a protective role in neurons against oxidative stress. These results suggest that lutein may be important in brain development. 
In this introductory chapter, we have presented the biochemical foundation of carotenoids. We have explained their most distinctive role in nature as antioxidants. Carotenoids are believed to be important as dietary micronutrients in humans since early life. They have been most widely studied in relation to human nutrition and health because they have shown to be beneficial in decreasing the risk of degenerative diseases.

The research presented in the following chapters studies the importance of carotenoids in early life. We have studied the levels of carotenoids in human milk, maternal serum and cord blood in a group of Peruvian women to identify the variations in concentrations and to understand the influence and relationships in carotenoid transport from mother to child. In addition we have studied the profile of fatty acids in human milk and the relationship between carotenoids and fatty acid composition in maternal milk. 
CHAPTER II: Carotenoids in maternal serum and cord blood

Dietary carotenoids have been extensively studied because of their association with health benefits. The study and measurements of carotenoids in human serum and plasma have been of great interest to help evaluate patterns of consumption, bioavailability, and potential for impact on human health and nutrition [47]. As described in the previous chapter, humans absorb carotenes and xanthophylls from the diet. But mechanisms and regulation of metabolism, distribution, tissue uptake and deposition of carotenoids in tissues are still not fully understood.

\subsection{Background}

\subsubsection{Analysis of carotenoids in human serum}

Khachik et al.[5] identified 34 different carotenoids, including 13 geometrical isomers, and 8 carotenoid metabolites in human plasma. As a result of the many carotenoids found in the circulating system, carotenoids in plasma and serum have been study for dietary clinical and epidemiological interventions [48]. Various factors including total dietary intake, efficiency of carotenoid to release from the food matrix, the efficiency of their absorption and metabolism, and rate of tissue uptake all influence the levels of carotenoids in serum. From among all the various carotenoids found in food (50 are commonly available in the human diet [49]), only six major carotenoids: $\beta$-carotene, $\alpha$-carotene, lycopene, $\beta$-cryptoxanthin, lutein, and zeaxanthin are found in serum at levels higher than their metabolites or than other acyclic carotenes $[5,25]$. As a consequence, these six carotenoids are the most studied and considered the most important carotenoids when evaluating human nutrition. 
High performance liquid chromatography (HPLC) has been the preferred method of analysis and isolation of carotenoids, and photodiode array detectors are the preferred detection method. Carotenoids are ideal for detection of UV-Vis absorbance due to their conjugated double bond structure, and the PDA detection method has provided the absorption spectra to facilitate carotenoid identification [13, 48]. Reversed-phase separation systems are most commonly used, with C18 columns and C30 columns having been proven useful for the demanding separation of carotenoids required for the identification and quantification [13]. HPLC-NMR has, in certain instances, been a useful technique for elucidation of cis-trans isomers and it has been applied to the determination of stereoisomers of zeaxanthin and lutein in retina [50].

\subsubsection{Carotenoids in maternal plasma and infant cord blood}

During pregnancy, carotenoids are transferred across the placenta to the fetus. Carotenoids are powerful antioxidants that can protect cells from oxidative stress. They are known to have an important role in human health and they are important in the diet during pregnancy and post-delivery, particularly $\beta$-carotene, which serves as major source of vitamin A. Oxidative stress has been suggested as a possible cause of human pregnancy-related disorders, such as preeclampsia [51]. Some studies found that women with preeclampsia generally have lower levels of $\beta$-carotene in plasma [52-54]. Although in a separate study conducted by Zhang et al. [51] this was not confirmed. Nutritional and environmental factors are sources of oxidative stress and are suspected causes of preterm birth or low birth weight. Premature birth and low birth weight are the most common cause of neonatal mortality [55]. There is no clear relationship between ROS 
induced oxidative stress and pregnancy related disorders [56], but the available information suggests that antioxidants including carotenoids are important to maintaining a health of the mother during pregnancy and contribute to improved fetal nourishment. Therefore, research is important to determine the maternal antioxidant micronutrient requirements.

Table 3. Studies showing carotenoid levels $(\mu \mathrm{mol} / \mathrm{L})$ in maternal plasma though pregnancy and cord blood of new born infants.

\begin{tabular}{|c|c|c|c|c|c|}
\hline & Yeum et al.[57] & $\begin{array}{c}\text { Oostenbrug et } \\
\text { al.[58] }\end{array}$ & $\begin{array}{c}\text { Oostenbrug et } \\
\text { al.[59] }\end{array}$ & $\dagger$ Kiely et al. [60] & Zhang et al.[51] \\
\hline $\begin{array}{c}1 \text { st } \\
\text { trimester }\end{array}$ & & $\begin{array}{c}\mathrm{L}=0.48 \pm 0.03 \\
\beta-C=0.30 \pm 0.02\end{array}$ & & & \\
\hline $\begin{array}{l}\text { 2nd } \\
\text { trimester }\end{array}$ & & $\begin{array}{c}L=0.57 \pm 0.03 \\
\beta-C=0.27 \pm 0.02\end{array}$ & & $\begin{array}{c}L+Z=0.46 \pm 0.20 \\
\beta-C r y=0.14 \pm 0.10 \\
\beta-C=0.23 \pm 0.10\end{array}$ & \\
\hline $\begin{array}{c}\text { 3rd } \\
\text { trimester }\end{array}$ & & $\begin{array}{c}L=0.65 \pm 0.04 \\
\beta-C=0.24 \pm 0.02\end{array}$ & $\begin{array}{c}L=0.64 \pm 0.04 \\
\beta-C=0.25 \pm 0.03\end{array}$ & & $\begin{array}{c}L=0.35 \pm 0.01 \\
Z=0.07 \pm 002 \\
\beta-C r y=0.43 \pm 0.03 \\
\beta-C=0.26 \pm 0.01\end{array}$ \\
\hline $\begin{array}{l}\text { Post- } \\
\text { partum }\end{array}$ & $\begin{array}{c}L=0.33 \pm 0.05 \\
Z=0.08 \pm 001 \\
\beta-C r y=0.59 \pm 0.12 \\
\beta-C=0.69 \pm 0.11\end{array}$ & $\begin{array}{c}L=0.64 \pm 0.04 \\
\beta-C=0.28 \pm 0.05\end{array}$ & $\begin{array}{c}L=0.65 \pm 0.04 \\
\beta-C=0.25 \pm 0.03\end{array}$ & & \\
\hline $\begin{array}{l}\text { Cord } \\
\text { Blood }\end{array}$ & $\begin{array}{c}L=0.05 \pm 0.01 \\
Z=0.01 \pm 0.00 \\
\beta-C r y=0.06 \pm 0.01 \\
\beta-C=0.03 \pm 0.01\end{array}$ & $\begin{array}{c}L=0.15 \pm 0.02 \\
\beta-C=0.03 \pm 0.00\end{array}$ & $\begin{array}{c}\mathrm{L}=0.13 \pm 0.03 \\
\beta-C=0.03 \pm 0.00\end{array}$ & $\begin{array}{c}L+Z=0.13 \pm 0.10 \\
\beta-C r y=0.04 \pm 0.03 \\
\beta-C=0.04 \pm 0.03\end{array}$ & \\
\hline
\end{tabular}

All values reported as mean \pm S.E.M., except $\uparrow$ mean \pm s.d.

$\mathrm{L}=$ lutein, $\mathrm{Z}=$ =eaxanthin, $\beta-\mathrm{C}=\beta$-carotene, $\beta$-Cry $=\beta$-cryptoxanthin.

Studies on $\beta$-carotene levels in cord blood have been reported [61-66], but there are only a few studies [57-60] that include lutein levels in addition to $\beta$-carotene in the new-born at time of birth. These studies compared the carotenoid levels in mother's plasma during pregnancy and postpartum with the levels found in cord blood in an effort to understand the maternal-fetal transfer of lipophilic molecules like carotenoids, retinol, 
and vitamins $\mathrm{A}$ and $\mathrm{E}$; in addition to assess their impact on pregnancy related disorders. An informative collection of data on cord blood and maternal carotenoid levels postpartum is shown in Table 3 (only studies including lutein were tabulated). In Table 3, we can see that carotenoid levels are lower in cord blood than in plasma. Almost all of these studies found a correlation between maternal serum and cord blood for lutein, but only a few studies showed a correlation between maternal and cord blood for $\beta$-carotene and $\beta$-cryptoxanthin.

Oostenbrug et al. [58] showed that lutein increased during pregnancy while $\beta$ carotene decreased. No significant change in the levels of carotenoids during the third trimester and post-partum was found by Oostenbrug. Some of the studies reported lutein to be the carotenoid found in the highest concentration in serum and cord blood, although they did not analyze for all carotenoids.

The objective of our research described in this chapter was to determine carotenoid levels in mothers' serum during post-partum and to correlate these levels with those in infants' cord blood serum to understand if there is a selective mobilization from mothers to their infants. Not many studies that address this question have been reported. Therefore, it is important to understand the role of carotenoids before and after delivery since they support the nutritional needs of the new born and contribute to optimal health in the mother. We are especially interested in lutein and zeaxanthin because they appear to play a central role in the development of the healthy new-born retina. 


\subsection{Research Methods}

\subsubsection{Study population}

The participants of the serum study were 74 Peruvian mothers and had a mean age was 26.3 years. All participants were in their first pregnancy and had no chronic health concerns. All participants had a full-term pregnancy (the deliveries occurred after the full 37 weeks of gestation). The volunteers for the cord blood study were 26 Peruvian women who were also participants of the serum analysis study. All volunteers were informed about the study and signed an IRB consent document (\#040411). Anthropometric measurements were also collected to estimate body mass index (BMI).

\subsubsection{Sample collection}

Cord blood samples were collected from 26 volunteers. Cord blood was taken by a trained technician following clamping and cutting of the cord. Blood was drawn from the umbilical vein using a vacutainer tube and was centrifuged to separate and collect the serum fraction.

Maternal venous blood samples $(10 \mathrm{~mL})$ were collected during the $4^{\text {th }}$ week after delivery from 74 volunteers. The drawn blood was then centrifuged to separate and collect the serum fraction.

All serum samples were stored at $-20^{\circ} \mathrm{C}$ for approximately one week at the study site. Samples were transferred weekly for storage at $-80^{\circ} \mathrm{C}$ until they were shipped overnight to our laboratory at Florida International University. Samples were shipped frozen on dried ice. 


\subsubsection{Chemicals}

Ethyl alcohol USP grade, 1-pentanol 99\%, hydrochloric acid, anhydrous diethyl ether, acetone, trimethylamine (TEA), anhydrous sodium sulfate, light petroleum ether, aluminum oxide powder (mesh 100, 99.9\%), potassium hydroxide, and butylated hydroxy toluene, USP grade (BHT) were all purchased from Sigma-Aldrich or Fisher Scientific. HPLC grade hexane, dichloromethane, methanol, and acetonitrile were purchased from Sigma-Aldrich and Fisher Scientific. Lutein, zeaxanthin standards were a gift from Industrial Organica S.A. $\beta$-carotene standard was purchased from Sigma-Aldrich and the $\beta$-cryptoxanthin standard was extracted from red bell peppers.

\subsubsection{Instruments}

System I: High pressure liquid chromatography (HPLC) system equipment was used for standard purification and consisted of a Waters 2690 coupled to a photodiode array detector (PDA) Waters 996. A Cary 100 ultraviolet-visible (UV-vis) spectrophotometer was used to determine the concentration of the carotenoid internal standard.

System II: The HPLC system used for carotenoid analysis was a Varian ProStar HPLC system with coupled to ProStar 335 photodiode array UV-visible detection system. A PhenomenexC-18 Ultracarb $3 \mu \mathrm{m}$ reversed-phase column was used with a mobile phase consisting of acetonitrile: methanol: triethylamine (85\%: $15 \%: 0.1 \% \mathrm{v} / \mathrm{v})$ and a flow rate of $1 \mathrm{~mL} / \mathrm{min}$ with a detection at $450 \mathrm{~nm}$. Chromatographic data were analyzed using the Varian software package. 


\subsubsection{Preparation of Standards:}

\section{A. Isolation of $\beta$-cryptoxanthin}

$\beta$-Cryptoxanthin isomers were extracted from red bell peppers following the procedure used by Breithaupt and Bamedi [67]. The peppers were crushed using a blender followed by multiple extractions with diethyl ether/light petroleum ether $(1: 1 \mathrm{v} / \mathrm{v})$ until the extracts had no color. The extract were combined and dried over anhydrous sodium sulfate. The resulting extract was dissolved in $200 \mathrm{~mL}$ of methanol, immediately after which $30 \mathrm{~mL}$ of methanolic $\mathrm{KOH}(30 \% \mathrm{w} / \mathrm{v})$ was added. The hydroxide solution was neutralized and the carotenoids were extracted in dicholoromethane. The dichloromethane solution was washed three times with $100 \mathrm{~mL}$ of water, dried over anhydrous sodium sulfate and evaporated using a rotary evaporator. The residue was dissolved in $3 \mathrm{~mL}$ of hexane in preparation for open column chromatography. The glass column used was $400 \mathrm{~mm}$ long and alumina was used as the stationary phase. $1 \mathrm{~mL}$ of the extracted carotenoids was separated using the column with hexane as the mobile phase. After the collection of the first band ( $\beta$-carotene), the mobile phase was changed to a mixture of hexane and acetone $(9: 1 \mathrm{v} / \mathrm{v})$ to elute a second band which contained the $\beta$ cryptoxanthin and its $c i s$-isomers.

B. Internal standard preparation:

Monopentyl lutein ether (MPL) was used as an internal standard for this study. The standard was prepared by conversion of pure lutein to the desired compound by the following procedure. $5 \mathrm{mg}$ of lutein was dissolved in $20 \mathrm{~mL}$ of 1-pentanol containing $1 \%$ $\mathrm{v} / \mathrm{v}$ of concentrated hydrochloric acid. The reaction was stirred in the dark for 1.5 hours. 
The resulting solution was extracted with a solution of dichloromethane. The product was concentrated and dissolved with ethanol prior to separation on reversed-phase HPLC. The monopentyl lutein ether component was collected from multiple injections, pooled and dried under $\mathrm{N}_{2}$. After reconstitution in acetonitrile the concentration of the resulting standard was determined using UV-visible spectrophotometry $\left(\varepsilon=1.45 \times 10^{5} \mathrm{moleL}^{-1} \mathrm{~cm}^{-1}\right)$.

\subsubsection{Serum and cord blood carotenoid extractions and analysis:}

The serum samples from the subjects were stored in $2 \mathrm{~mL}$ polypropylene vials, labeled, and stored at $-80^{\circ} \mathrm{C}$ in the freezer before shipment to our laboratory. The carotenoids were extracted according to a previous published technique [68]. Each sample was thawed at room temperature and vortexed briefly to ensure homogeneity. An aliquot of $400 \mu \mathrm{L}$ of serum was placed in a glass tube, 135ng-170ng of MPL was added, and $4 \mathrm{~mL}$ of a mixture of ethanol/deionized water $(1: 1)$ was added to the tube. The tube was vortexed for 30 seconds and carotenoids were extracted four times with $4 \mathrm{~mL}$ of hexane. For each extraction the tube was vortexed and centrifuged for 5 minutes. The top hexane layer was removed and combined in a $2 \mathrm{~mL}$ vial. The resulting aliquot obtained was dried under nitrogen gas flow. The sample was re-dissolved in $80 \mu \mathrm{L}$ of ethanol and transferred to a vial for HPLC injection. HPLC system I was used to separate and quantify the carotenoids in these samples.

\subsubsection{Statistical Analysis}

Data were analyzed using Statistical Packages for Social Sciences version 21.0

(SPSS In c. Chicago, IL, USA). Statistical methods were employed to test our 
hypotheses; a two-way ANOVA test was conducted for a group of 26 subjects that donated milk samples during the three consecutive weeks of the study.

\subsection{Results}

\subsubsection{Characterization of the standards}

The UV-Visible spectra shown in Figure 16 were obtained for the $\beta$-cryptoxanthin and standards prepared as described above. The absorbance spectrum of the monomethyl lutein internal standard in Figure 17A is the same as that of lutein $\left(\lambda_{\max }: 445 \mathrm{~nm}\right.$ and 474 $\mathrm{nm}$ ). The concentration of the standard was determined based on the absorbance at 450nm,. The spectrum in Figure 17B corresponds to $\beta$-cryptoxanthin isolated from red bell peppers, having $\lambda_{\max }$ at $448 \mathrm{~nm}$ and $470 \mathrm{~nm}$, consistent with the literature.(CitationBritton)

A

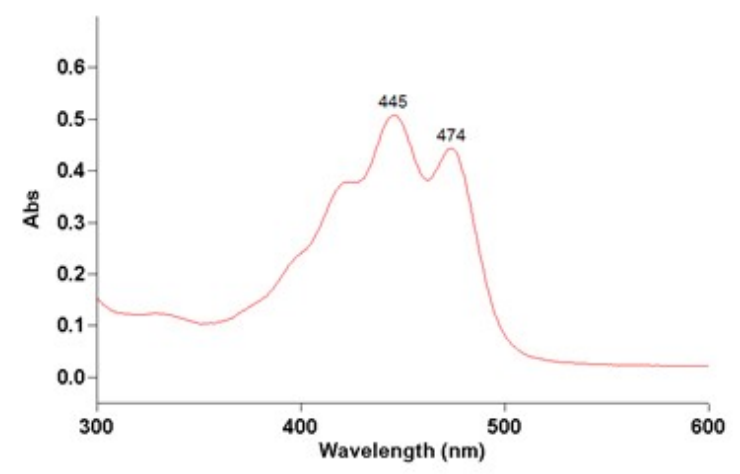

B

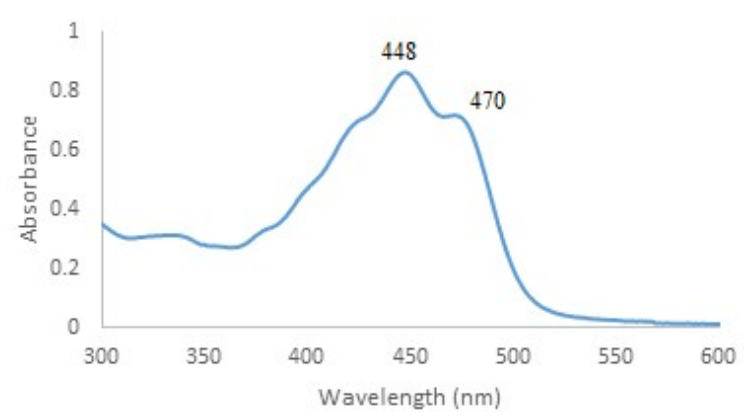

Figure 17. UV-visible spectra of standards used for analysis and identification. A)Monopentyl lutein and B) $\beta$-cryptoxanthin. 


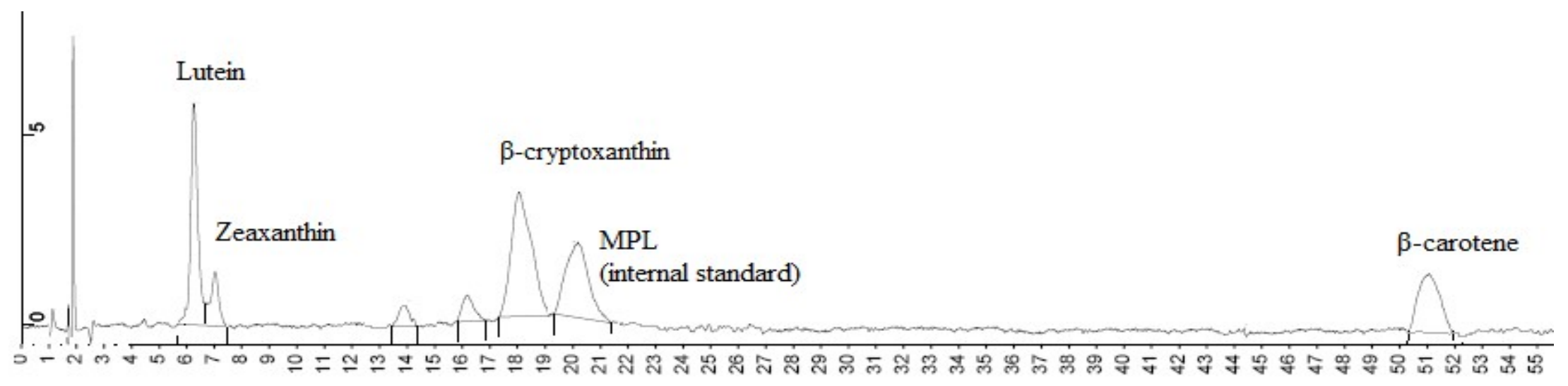

Figure 18. Carotenoid standards' chromatogram.

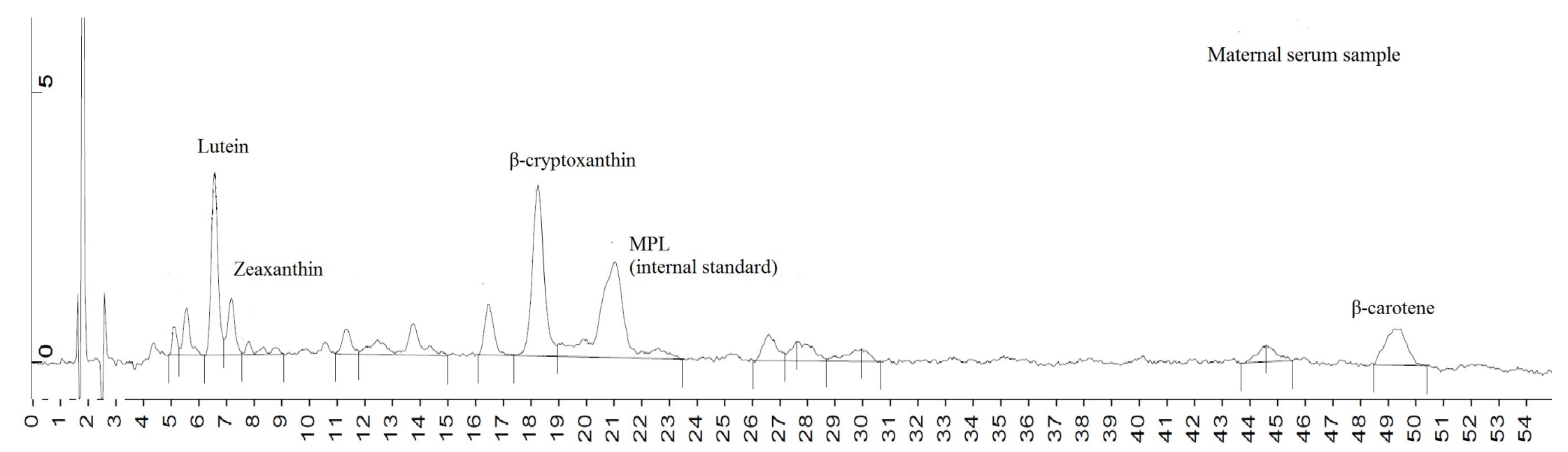

Figure 19. Example of mother's serum chromatogram 


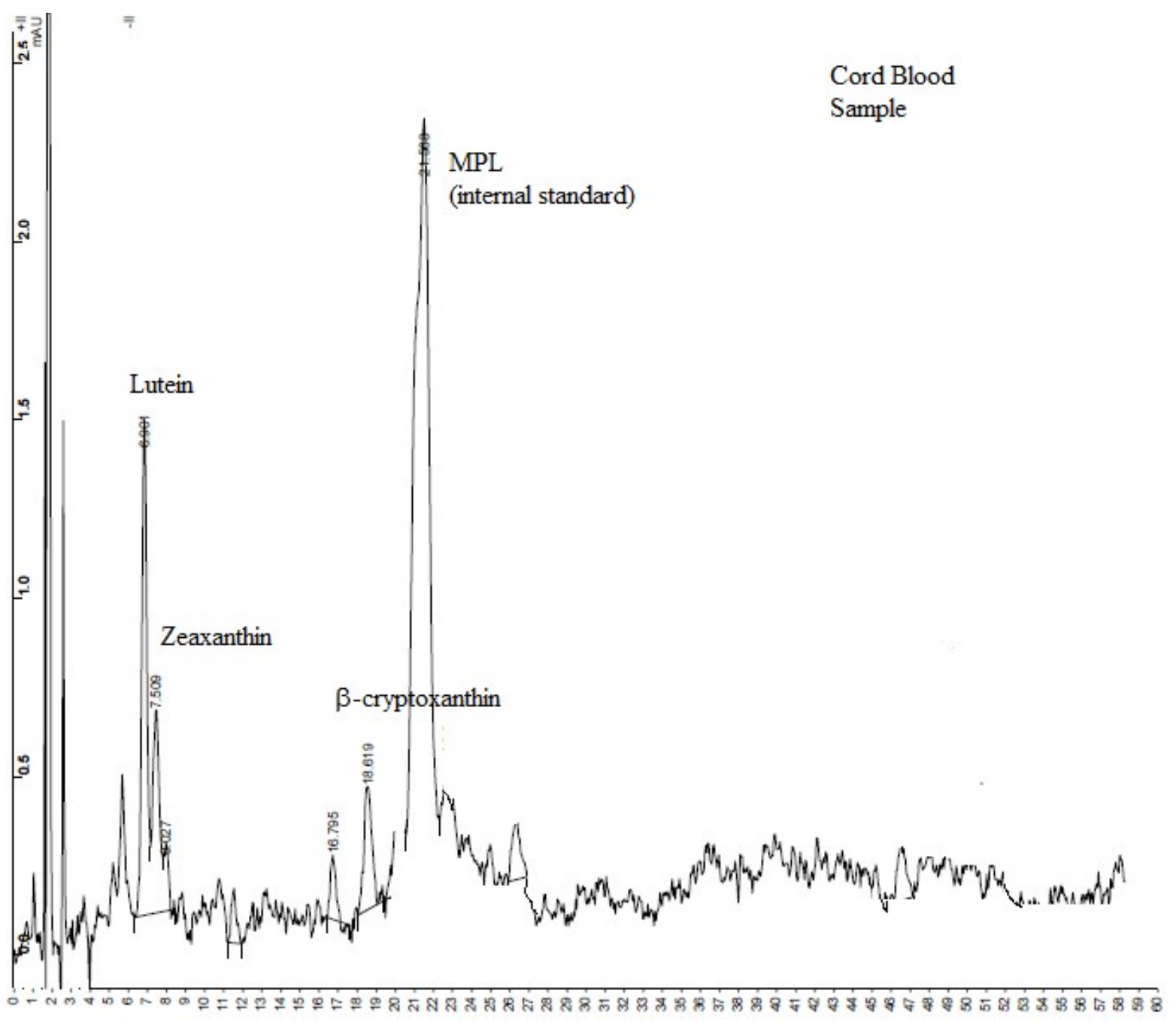

Figure 20. A typical chromatogram of the carotenoids extracted from cord blood. 
Figure 18 shows a chromatogram of the combined carotenoids showing their a typical separation. Figures 19 and 20 correspond to examples of serum and cord blood analyzed in the present study.

Tables 4-5 show the results for all the samples analyzed to measure levels of carotenoids content in serum and cord blood. Our data on carotenoid concentrations are consistent with previous reported values $[51,57,69]$

Table 4. Results from the analysis of carotenoids in cord blood for all subjects, amounts in $\mu \mathrm{mol} / \mathrm{L}$.

\begin{tabular}{|ccccc|ccccc|}
\hline Subject & Lutein & Zea & $\boldsymbol{\beta}$-cry & $\mathbf{L} / \mathbf{Z}$ & Subject & Lutein & Zea & $\boldsymbol{\beta}$-cry & $\mathbf{L} / \mathbf{Z}$ \\
$\mathbf{N}$ & $\mathbf{2 6}$ & $\mathbf{2 6}$ & $\mathbf{1 8}$ & $\mathbf{2 6}$ & & & & & \\
\hline 2701 & 0.08 & 0.04 & 0.04 & 2.12 & 2743 & 0.13 & 0.05 & 0.07 & 2.54 \\
2705 & 0.07 & 0.04 & $* *$ & 2.01 & 4709 & 0.13 & 0.05 & 0.09 & 2.52 \\
2713 & 0.07 & 0.02 & 0.07 & 3.92 & 4712 & 0.06 & 0.04 & 0.05 & 1.39 \\
2716 & 0.09 & 0.04 & 0.13 & 2.00 & 4716 & 0.05 & 0.02 & 0.04 & 2.09 \\
2717 & 0.08 & 0.05 & 0.07 & 1.76 & 4718 & 0.02 & 0.02 & $* *$ & 1.12 \\
2724 & 0.11 & 0.08 & 0.05 & 1.45 & 4725 & 0.05 & 0.02 & 0.06 & 2.00 \\
2727 & 0.08 & 0.04 & 0.04 & 1.70 & 4726 & 0.06 & 0.03 & 0.03 & 2.23 \\
2736 & 0.08 & 0.03 & $* *$ & 2.93 & 4729 & 0.05 & 0.03 & 0.03 & 1.51 \\
2737 & 0.10 & 0.02 & 0.10 & 3.92 & 4730 & 0.04 & 0.02 & $* *$ & 1.74 \\
2738 & 0.05 & 0.02 & $* *$ & 3.15 & 4731 & 0.08 & 0.06 & 0.06 & 1.43 \\
2739 & 0.11 & 0.04 & 0.17 & 2.72 & 4738 & 0.04 & 0.02 & $* *$ & 1.73 \\
2741 & 0.08 & 0.04 & 0.06 & 2.21 & 4739 & 0.07 & 0.05 & 0.62 & 1.46 \\
2742 & 0.06 & 0.03 & $* *$ & 2.16 & 4741 & 0.07 & 0.04 & $* *$ & 1.84 \\
\hline
\end{tabular}

**not detected 
Table 5. Results of analysis of carotenoids in mother's serum for all subjects, amounts in $\mu \mathrm{mol} / \mathrm{L}$.

\begin{tabular}{|c|c|c|c|c|c|c|c|c|c|c|c|}
\hline $\begin{array}{c}\text { Subject } \\
\mathbf{N} \\
\end{array}$ & $\begin{array}{c}\text { Lutein } \\
74 \\
\end{array}$ & $\begin{array}{c}\text { Zea } \\
74 \\
\end{array}$ & $\begin{array}{c}\beta \text {-cry } \\
74 \\
\end{array}$ & $\begin{array}{c}\beta \text {-car } \\
61\end{array}$ & $\begin{array}{c}L / Z \\
74 \\
\end{array}$ & Subject & Lutein & Zea & $\beta$-cry & $\beta$-car & $\mathbf{L} / \mathbf{Z}$ \\
\hline 2701 & 0.47 & 0.09 & 0.15 & $* *$ & 3.02 & 2741 & 0.30 & 0.14 & 0.46 & 0.17 & 2.19 \\
\hline 2702 & 0.17 & 0.07 & 0.09 & $* *$ & 2.56 & 2742 & 0.21 & 0.08 & 0.23 & $* *$ & 2.54 \\
\hline 2703 & 0.44 & 0.17 & 0.07 & 0.12 & 2.65 & 2743 & 0.41 & 0.12 & 0.67 & 0.24 & 3.53 \\
\hline 2704 & 0.55 & 0.16 & 0.38 & $* *$ & 3.38 & 4701 & 0.28 & 0.12 & 0.13 & 0.18 & 2.33 \\
\hline 2705 & 0.37 & 0.15 & 0.19 & $* *$ & 2.47 & 4702 & 0.35 & 0.16 & 0.12 & 0.13 & 2.21 \\
\hline 2706 & 0.19 & 0.11 & 0.40 & 0.07 & 1.81 & 4704 & 0.22 & 0.13 & 0.14 & 0.16 & 1.71 \\
\hline 2707 & 0.25 & 0.08 & 0.36 & 0.36 & 3.03 & 4705 & 0.21 & 0.08 & 0.15 & 0.12 & 2.65 \\
\hline 2708 & 0.22 & 0.10 & 0.23 & 0.10 & 2.31 & 4706 & 0.12 & 0.06 & 0.10 & $* *$ & 2.04 \\
\hline 2709 & 0.20 & 0.09 & 0.46 & 0.13 & 2.22 & 4708 & 0.22 & 0.10 & 0.11 & $* *$ & 2.33 \\
\hline 2710 & 0.48 & 0.15 & 0.13 & 0.19 & 3.25 & 4709 & 0.33 & 0.16 & 0.31 & 0.62 & 2.06 \\
\hline 2712 & 0.24 & 0.11 & 0.10 & 0.33 & 2.11 & 4710 & 0.23 & 0.07 & 0.08 & 0.45 & 3.33 \\
\hline 2713 & 0.26 & 0.10 & 0.29 & $* *$ & 2.51 & 4712 & 0.15 & 0.08 & 0.23 & 0.20 & 1.84 \\
\hline 2714 & 0.16 & 0.09 & 0.26 & 0.36 & 1.87 & 4713 & 0.21 & 0.10 & 0.45 & 0.23 & 2.15 \\
\hline 2715 & 0.22 & 0.18 & 1.83 & 0.29 & 1.22 & 4714 & 0.24 & 0.08 & 0.20 & 0.56 & 3.06 \\
\hline 2716 & 0.17 & 0.06 & 0.43 & 0.23 & 3.00 & 4715 & 0.14 & 0.08 & 0.23 & 0.45 & 1.86 \\
\hline 2717 & 0.25 & 0.09 & 0.16 & 0.23 & 2.64 & 4716 & 0.33 & 0.18 & 1.30 & 0.29 & 1.84 \\
\hline 2718 & 0.18 & 0.09 & 0.29 & 0.15 & 2.05 & 4717 & 0.16 & 0.10 & 0.59 & 0.90 & 1.68 \\
\hline 2719 & 0.22 & 0.12 & 0.69 & 0.28 & 1.79 & 4718 & 0.13 & 0.05 & 0.18 & 0.23 & 2.67 \\
\hline 2720 & 0.25 & 0.10 & 0.32 & 0.51 & 2.57 & 4719 & 0.13 & 0.09 & 0.09 & 0.21 & 1.51 \\
\hline 2721 & 0.16 & 0.05 & 0.22 & $* *$ & 2.90 & 4720 & 0.20 & 0.10 & 0.15 & 0.17 & 2.01 \\
\hline 2722 & 0.21 & 0.12 & 0.51 & 0.36 & 1.82 & 4721 & 0.12 & 0.08 & 0.75 & 0.00 & 1.55 \\
\hline 2723 & 0.46 & 0.14 & 0.44 & 1.10 & 3.22 & 4723 & 0.28 & 0.12 & 0.63 & 0.37 & 2.30 \\
\hline 2724 & 0.20 & 0.06 & 0.29 & 0.16 & 3.24 & 4724 & 0.19 & 0.09 & 0.53 & 0.20 & 2.24 \\
\hline 2725 & 0.24 & 0.07 & 0.30 & 0.24 & 3.30 & 4725 & 0.40 & 0.12 & 0.88 & 0.36 & 3.32 \\
\hline 2726 & 0.69 & 0.26 & 0.99 & 0.21 & 2.65 & 4726 & 0.32 & 0.12 & 0.55 & $* *$ & 2.66 \\
\hline 2727 & 0.29 & 0.10 & 0.48 & 0.19 & 2.97 & 4727 & 0.41 & 0.16 & 0.58 & 0.05 & 2.50 \\
\hline 2728 & 0.30 & 0.13 & 0.79 & 0.15 & 2.34 & 4728 & 0.31 & 0.13 & 0.66 & 0.26 & 2.44 \\
\hline 2729 & 0.39 & 0.17 & 0.28 & $* *$ & 2.23 & 4729 & 0.17 & 0.08 & 0.46 & 0.28 & 2.03 \\
\hline 2730 & 0.65 & 0.27 & 0.46 & 0.27 & 2.43 & 4730 & 0.24 & 0.08 & 0.09 & $* *$ & 3.16 \\
\hline 2732 & 0.16 & 0.07 & 0.21 & 0.14 & 2.34 & 4731 & 0.36 & 0.17 & 0.85 & 0.21 & 2.07 \\
\hline 2733 & 0.24 & 0.05 & 0.12 & $* *$ & 5.00 & 4732 & 0.23 & 0.10 & 0.32 & 0.17 & 2.36 \\
\hline 2735 & 0.40 & 0.17 & 1.31 & 0.43 & 2.35 & 4733 & 0.12 & 0.05 & 0.23 & 0.17 & 2.35 \\
\hline 2736 & 0.25 & 0.14 & 0.25 & 0.23 & 1.85 & 4736 & 0.36 & 0.08 & 0.13 & 0.12 & 4.57 \\
\hline 2737 & 0.33 & 0.13 & 0.44 & 0.31 & 2.55 & 4738 & 0.14 & 0.07 & 0.22 & 0.13 & 2.14 \\
\hline 2738 & 0.34 & 0.19 & 0.36 & 0.14 & 1.79 & 4739 & 0.16 & 0.08 & 0.45 & 0.16 & 1.87 \\
\hline 2739 & 0.34 & 0.13 & 0.57 & 0.41 & 2.64 & 4740 & 0.30 & 0.12 & 0.22 & 0.18 & 2.58 \\
\hline 2740 & 0.37 & 0.13 & 0.72 & 0.12 & 2.79 & 4741 & 0.29 & 0.13 & 0.32 & 0.27 & 2.19 \\
\hline
\end{tabular}

**not detected 
In maternal serum, $\beta$-carotene was detected in the majority of the subjects $(81 \%)$, however in cord blood, $\beta$-carotene was either not detected or barely detected, therefore we chose to not to include $\beta$-carotene in the results for cord blood. $\beta$-cryptoxanthin was detected in cord blood in $69 \%$ of the subjects as well as in all maternal serum samples.

Table 6 shows the correlations found in serum for lutein, zeaxanthin, and $\beta$ cryptoxanthin; $\beta$-carotene did not show any significant correlation with the rest of the carotenoids. In cord blood, only lutein and zeaxanthin correlate; $\beta$-cryptoxanthin did not correlate with either lutein or zeaxanthin.

Table 6. Correlations ( $\mathrm{r}$ ) of carotenoids in serum

\begin{tabular}{|c|ccc|}
\hline $\mathrm{N}=74$ & lutein & zeaxanthin & $\beta$-cryptoxanthin \\
\hline lutein & -- & $0.81^{* *}$ & $0.25^{*}$ \\
zeaxanthin & $0.81^{* *}$ & -- & $0.48^{* *}$ \\
cryptoxanthin & $0.25^{*}$ & $0.48^{* *}$ & -- \\
\hline
\end{tabular}

** Correlation is significant at the 0.01 level (2-tailed).

* Correlation is significant at the 0.05 level (2-tailed).

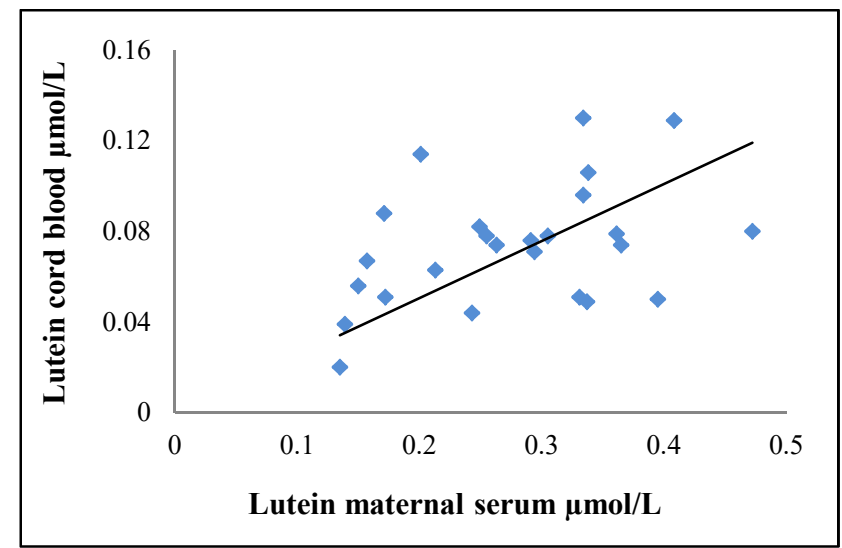

Figure 21. Correlation of lutein in maternal serum and cord blood $(\mathrm{r}=0.395, \mathrm{p}=0.046$, $\alpha=0.05)$ 


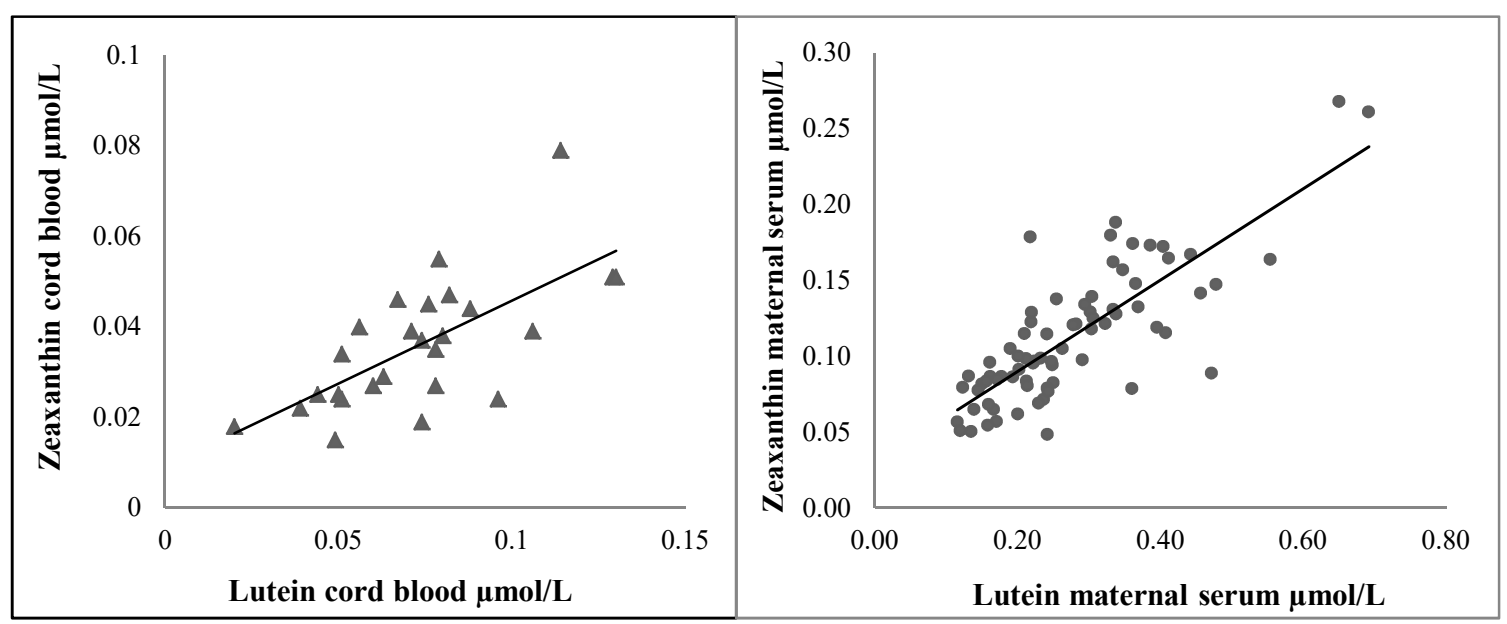

Figure 22 Correlation of lutein and zeaxanthin in (A) cord blood (r=0.69, $\mathrm{p}<0.001)$ (B) maternal serum $(r=0.81, \mathrm{p}<0.001, \alpha=0.05)$.

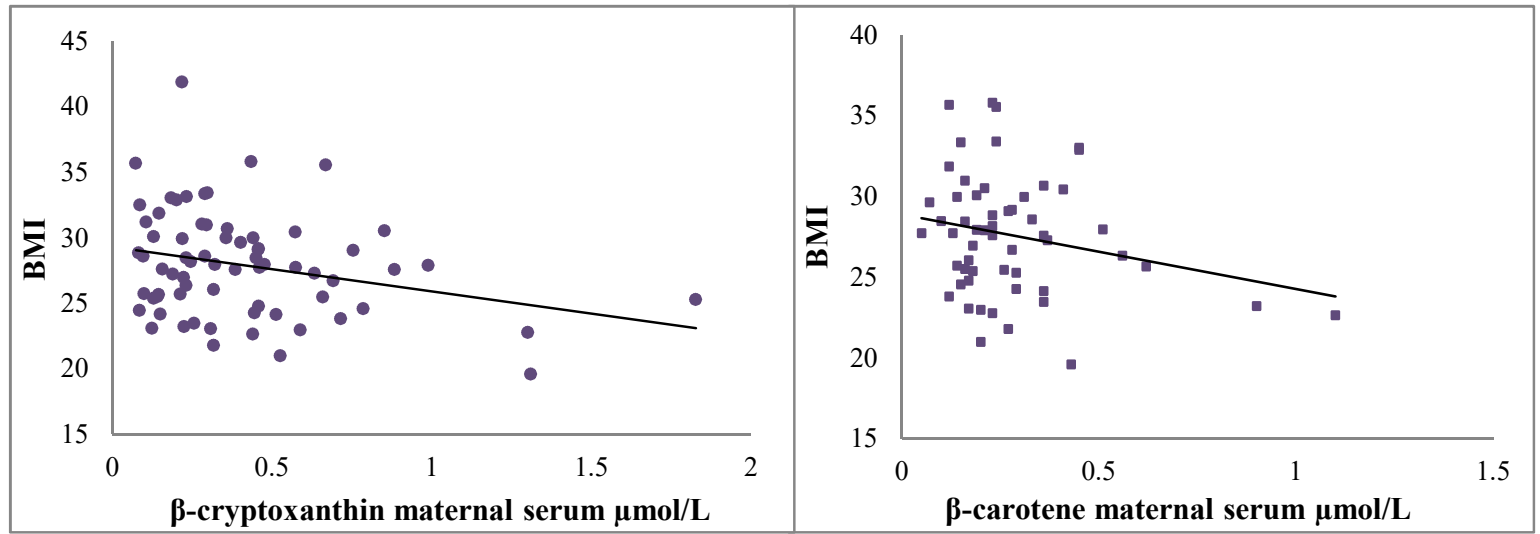

Figure 23 Correlation of BMI to maternal serum of $(A) \beta$-cryptoxanthin $(r=-0.28$, $\mathrm{p}=0.02)(\mathrm{B}) \beta$-carotene $(\mathrm{r}=-0.26, \mathrm{p}=0.055, \alpha=0.05)$.

Figures 21-23 show the different correlations found for carotenoids in maternal serum and cord blood. Only lutein shows a correlation between cord blood and maternal serum $(\mathrm{r}=0.395, \mathrm{p}=0.046, \mathrm{~N}=26)$, Figure 21 , while zeaxanthin and $\beta$-cryptoxanthin did not. Table 7 shows the correlations found between BMI and pro-vitamin A carotenoids, lutein and zeaxanthin did not correlate with BMI. 
Table 7. Correlation between BMI and pro-vitamin A carotenoids in maternal serum.

\begin{tabular}{|c|cc|}
\hline & $\begin{array}{c}\beta \text {-cryptoxanthin } \\
\mathrm{N}=69\end{array}$ & $\begin{array}{c}\beta \text {-carotene } \\
\mathrm{N}=56\end{array}$ \\
\hline \multirow{2}{*}{ BMI } & $\mathrm{r}=-0.272$ & $\mathrm{r}=-0.258$ \\
& $\mathrm{p}=0.024$ & $\mathrm{p}=0.055$ \\
\hline
\end{tabular}

Table 8. Lutein and zeaxanthin concentrations ( $\mu \mathrm{mol} / \mathrm{L})$ in cord blood according to newborn's sex.

\begin{tabular}{|l|cc|}
\hline & $\begin{array}{c}\text { Female } \\
\mathrm{N}=15\end{array}$ & $\begin{array}{c}\text { Male } \\
\mathrm{N}=10\end{array}$ \\
\hline Lutein & $0.07 \pm 0.02$ & $0.07 \pm 0.03$ \\
Zeaxanthin & $0.04 \pm 0.01$ & $0.03 \pm 0.01$ \\
\hline
\end{tabular}

After dividing the cord blood data by newborn's sex (Table 8), we did not observe any significant difference for the levels of lutein or carotenoids between the groups.

\subsection{Discussion and Conclusions}

Our study measured the carotenoid levels in cord blood and in maternal serum during the first month post-partum and investigated the relationships between these values. Tables 9 and 10 summarize these data averaged for all samples. Our results are within the ranges reported by previous investigations, see Table 2. The only study of Peruvian women's serum was reported by Zhang et al. [51] during the third trimester of gestation. The carotenoids levels in that study were reported to be $L=0.35 \pm 0.01, Z=0.07$ $\pm 002, \beta-C r y=0.43 \pm 0.03$, and $\beta-C=0.26 \pm 0.01$. The values reported here (see Table 9) for lutein and $\beta$-cryptoxanthin are lower in comparison. It should be recognized that our 
results were taken during the $4^{\text {th }}$ week post-partum, which could affect the levels reflected in serum attributable to micronutrients transfer to milk during the lactation period. In addition, the dietary intake that varies by subject will change the concentration of carotenoids in maternal serum, and our data are from a smaller sample than in the study by Zhang.

Table 9. Average carotenoids levels $(\mu \mathrm{mol} / \mathrm{L})$ in mother's serum.

\begin{tabular}{|c|cccccc|}
\hline \multicolumn{7}{|c|}{ Mothers' serum } \\
\hline & N & Min & Max & Mean & Std. Dev. & Median \\
\hline Lutein & 74 & 0.12 & 0.69 & 0.27 & 0.12 & 0.24 \\
Zeaxanthin & 74 & 0.05 & 0.27 & 0.11 & 0.04 & 0.10 \\
$\beta$-cryptoxanthin & 74 & 0.07 & 1.82 & 0.40 & 0.32 & 0.30 \\
$\beta$-carotene & 60 & 0.05 & 1.10 & 0.27 & 0.18 & 0.21 \\
L/Z & 74 & 1.22 & 5.00 & 2.47 & 0.64 & 2.35 \\
BMI & 69 & 19.61 & 41.89 & 27.94 & 4.00 & 27.74 \\
\hline
\end{tabular}

Table 10. Average carotenoids levels $(\mu \mathrm{mol} / \mathrm{L})$ in cord blood.

\begin{tabular}{|c|cccccc|}
\hline \multicolumn{7}{|c|}{ Cord Blood } \\
\hline & N & Min & Max & Mean & Std. Dev. & Median \\
\hline Lutein & 26 & 0.02 & 0.13 & 0.07 & 0.03 & 0.07 \\
Zeaxanthin & 26 & 0.02 & 0.08 & 0.04 & 0.01 & 0.04 \\
$\beta$-cryptoxanthin & 18 & 0.03 & 0.61 & 0.10 & 0.13 & 0.06 \\
L/Z & 26 & 1.12 & 3.92 & 2.14 & 0.72 & 2.01 \\
\hline
\end{tabular}

As anticipated based on previous reports [57, 60], for the paired results (see Table 11), comparing cord blood to maternal serum shows that carotenoid concentrations are significantly lower $(\mathrm{p}<0.001)$ in cord blood than in maternal serum for lutein, zeaxanthin, and $\beta$-cryptoxanthin. In cord blood, the levels of lutein, zeaxanthin, and $\beta$-cryptoxanthin 
were found on average to be $26.3 \%, 32.1 \%$, and $19.5 \%$ that of maternal serum. Lutein was the most abundant carotenoid found in cord blood in more than $87 \%$ of the cases, but in maternal serum $\beta$-cryptoxanthin was the most abundant carotenoid in $60 \%$ of the cases. Similar results were observed by Yeum et al. [57] in their study. We found that $\beta$ carotene levels are barely detected in cord blood; other investigators have found low levels of $\beta$-carotene in cord blood. It has been proposed by Dimenstein et al. [64] that this may reflect enhanced metabolism of $\beta$-carotene by the embryo or reduced placental transport of $\beta$-carotene in comparison to other carotenoids. A comparison of the ratio of lutein to zeaxanthin in cord blood (L: $Z=2.14 \pm 0.72)$ to that in maternal serum (L: $Z=$ $2.49 \pm 0.52)$ does not show any statistically significant variation. On this basis we conclude that lutein is not preferably uptake by the cord blood or actively transported to the fetus during the gestation period.

Table 11. Carotenoid concentrations $(\mu \mathrm{mol} / \mathrm{L})$ for maternal and cord blood in paired samples.

\begin{tabular}{|c|ccc|}
\hline & $\mathrm{N}$ & Cord blood & Maternal Serum \\
\hline Lutein & 26 & $0.07 \pm 0.03^{*}$ & $0.28 \pm 0.09$ \\
Zeaxanthin & 26 & $0.04 \pm 0.01^{*}$ & $0.11 \pm 0.04$ \\
B-cryptoxanthin & 18 & $0.10 \pm 0.13^{*}$ & $0.50 \pm 0.29$ \\
L/Z & 26 & $2.14 \pm 0.72$ & $2.49 \pm 0.52$ \\
\hline
\end{tabular}

*significantly different $(\mathrm{p}<0.001)$

There was a significant correlation of lutein $(\mathrm{r}=0.41, \mathrm{p}=0.04)$ between serum and cord blood, see Figure 20. This correlation has been reported before, while the correlation between other carotenoids, such as zeaxanthin and pro-vitamin A carotenoids, has not been consistently reported. In addition, lutein correlates highly with zeaxanthin 
in both serum $(\mathrm{r}=0.81, \mathrm{p}<0.001)$ and cord blood $(\mathrm{r}=0.69, \mathrm{p}<0.001)$, see Figure 22, whereas $\beta$-cryptoxanthin is correlated with lutein $(\mathrm{r}=0.25, \mathrm{p}=0.03)$ and zeaxanthin $(\mathrm{r}=0.48, \mathrm{p}<0.001)$ only in serum and not in cord blood.

It was also observed that in maternal serum, $\beta$-carotene concentrations do not correlate with any other carotenoid measured in the study. Interestingly in maternal serum, body mass index was found to inversely associate with pro-vitamin A carotenoids, $\beta$-cryptoxanthin $(\mathrm{r}=-0.28, \mathrm{p}=0.02)$, and $\beta$-carotene $(\mathrm{r}=-0.26, \mathrm{p}=0.055)$, see Table 7 and Figure 23, but did not correlate with lutein or zeaxanthin. A number of other investigations [70-72] have also found an inverse association between BMI and carotenoid concentrations in serum. In all of the cases, $\beta$-carotene was found to be correlated with BMI. Andersen et al.[71] have suggested that this relationship, between BMI and carotenoids, may be explained by three factors. First, the correlations could reflect dietary and lifestyle factors that are particular to each individual. Second, since carotenoids are lipophilic, they are distributed between blood and fat tissue. Thus, carotenoids are lower in individuals with higher BMI because a relatively larger proportion is solubilized in adipose. A third possible explanation is that individuals with more adipose tissue may experience greater oxidative stress, leading to degradation of carotenoids at a greater rate. In addition, $\beta$-carotene conversion to vitamin A depends on several factors, one which is BMI [73]. This relationship is established by adipose tissue being a site of $\beta$-carotene accumulation and $\mathrm{BCMO} 1$ expression. Therefore, the inverse relationship between $\beta$-carotene and BMI could reflect the partition of pro-vitamin A carotenoids between plasma and adipose tissue. Because of these factors, the 
concentration of $\beta$-carotene is greater in serum when BMI is low, but with a higher BMI it would be concentrated primarily within the adipose tissue. We cannot say the same about $\beta$-cryptoxanthin because the conversion of $\beta$-cryptoxanthin to vitamin $\mathrm{A}$ is lower; thus additional studies are needed to address this finding. Lastly, in this study we did not find the lutein levels in cord blood to be sex dependent as suggested in a previous investigation [74] of lutein levels in cord blood.

In conclusion, this is the first time levels and relationships of carotenoids between mothers and new-born were study in this particular population. Our study found an inverse correlation between BMI and $\beta$-cryptoxanthin in maternal serum. It is informative to address the carotenoid maternal nutrition status in Peruvian women. Carotenoids in cord blood are lower in concentration than those in maternal serum. Lutein was also found to be a major component in both maternal serum and cord blood. Lutein showed a positive correlation between maternal serum and cord blood, and the ratio of lutein to zeaxanthin was similar in both. One possible explanation for this result could be that the levels in maternal serum measured were depleted because of the preferential transfer of this nutrient to breast milk to maintain the nourishment required by the infant during the same period. Consequently, we cannot confirm that placental lutein transfer is favored during early life. More research is needed to help understand this relationship. 
CHAPTER III: Fatty acids in human milk

Human milk is the sole source of nutrition for infants for the first six months of life. Milk is a complex fluid that contains carbohydrates, salts in solution, milk proteins (casein) in colloidal dispersion, cells, cellular debris, and lipids, mostly in emulsified globules [75]. It also contains biological components such as antibodies, enzymes, and hormones that contribute to the development of the digestive tract and the immune system in the new born [76]. Macronutrients such as proteins, fat, and carbohydrates, are the major components present in human milk while micronutrients comprise less than $0.5 \%$ (vitamins, carotenoids, retinol, and some minerals).

Table 12. Multiple functions of the major nutrients of human milk (adapted from [77] p. 70). LC-PUFA = long chain polyunsaturated fatty acids; FFA = free fatty acids, produced from triglycerides during fat digestion in the stomach and intestine.

\begin{tabular}{|c|c|c|c|}
\hline \multicolumn{2}{|l|}{ Macronutrients } & Amount & Function \\
\hline Protein & $\begin{array}{c}\text { IgA } \\
\text { IgM } \\
\text { IgG } \\
\text { Lactoferrin } \\
\text { Lysozyme } \\
\alpha \text {-Lactalbumin } \\
\text { Casein }\end{array}$ & $\begin{array}{c}50-100 \mathrm{mg} / \mathrm{dL} \\
2 \mathrm{mg} / \mathrm{dL} \\
1 \mathrm{mg} / \mathrm{dL} \\
100-300 \mathrm{mg} / \mathrm{dL} \\
5-25 \mathrm{mg} / \mathrm{dL} \\
200-300 \mathrm{mg} / \mathrm{dL} \\
200-300 \mathrm{mg} / \mathrm{dL}\end{array}$ & $\begin{array}{l}\text { Immune protection } \\
\text { Immune protection } \\
\text { Immune protection } \\
\text { Anti-infective, iron carrier } \\
\text { Anti-infective } \\
\text { Ion carrier }(\mathrm{Ca}+) \text { part of lactose synthase } \\
\text { Ion carrier, inhibits microbial adhesion to } \\
\text { mucosal membranes }\end{array}$ \\
\hline Carbohydrate & $\begin{array}{c}\text { Lactose } \\
\text { Oligosaccharides } \\
\text { Glycoconjugates }\end{array}$ & $\begin{array}{l}6.5-7.3 \mathrm{~g} / \mathrm{L} \\
1.0-1.5 \mathrm{~g} / \mathrm{L} \\
-\end{array}$ & $\begin{array}{l}\text { Energy source } \\
\text { Microbial ligands } \\
\text { Microbial and viral ligands }\end{array}$ \\
\hline Fat & $\begin{array}{l}\text { Triglyceride } \\
\text { LC-PUFA } \\
\text { FFA }\end{array}$ & $3.0-4.5 \mathrm{~g} / \mathrm{L}$ & $\begin{array}{l}\text { Energy source } \\
\text { Essential for brain and retinal development } \\
\text { and for infant growth } \\
\text { Anti-infective Fat }\end{array}$ \\
\hline
\end{tabular}


The lipids present in human milk comprise $\sim 3-5 \%$ by mass and occur principally as globules emulsified in the aqueous phase (87\%) of the fluid. Lipids are present as triacylglycerols (TG) ( $98 \%)$, phospholipids ( $\sim .8 \%)$, and cholesterol ( 0.5\%) [77].

This chapter provides an overview of the general concepts of fatty acids and on fatty acids in human milk and includes our results of the analysis of human milk fat in 74 Peruvian women. The data include measurement of the amounts of fatty acids from the triglycerides present in milk. A discussion of the influence of the mother's diet on the fatty acid profiles of human milk is also included.

\subsection{Background}

\subsubsection{Fatty acid structure and functions}

Fatty acids are hydrocarbons composed of four to thirty six carbon atoms with a methyl group at the (omega, $\omega$ ) end of the molecule and a carboxyl group at the other end. The carbon chain can be short (2-4 carbons), medium (6-12 carbons), long (14-18 carbons), or very long (derived from 18 carbon or more) [78]. In naming, the carbon atom adjacent to the carboxyl group is designated as the $\alpha$ carbon, and the following carbons in the chain are labeled sequentially with the Greek letters $\beta, \gamma, \delta$, etc. The Latin letter " $n$ " is regularly used, instead of the Greek $\omega$, when indicating the position of any double bonds as counted from the methyl end, see Figure 1. The nomenclature also allows the location of double bonds to be referenced to the carboxyl group [79]. A short formula that is conventionally used to designate the identity of fatty acids. In the short formula the carbon number is followed by the number of double bonds present and the 
location of the double bonds is assigned relative to the $\omega$ carbon atom. For example, in linoleic acid which has 18 carbon atoms and two double bonds the final double bond is in the omega- $6(n-6)$ position and the fatty acid is designated $18: 2 n-6$.

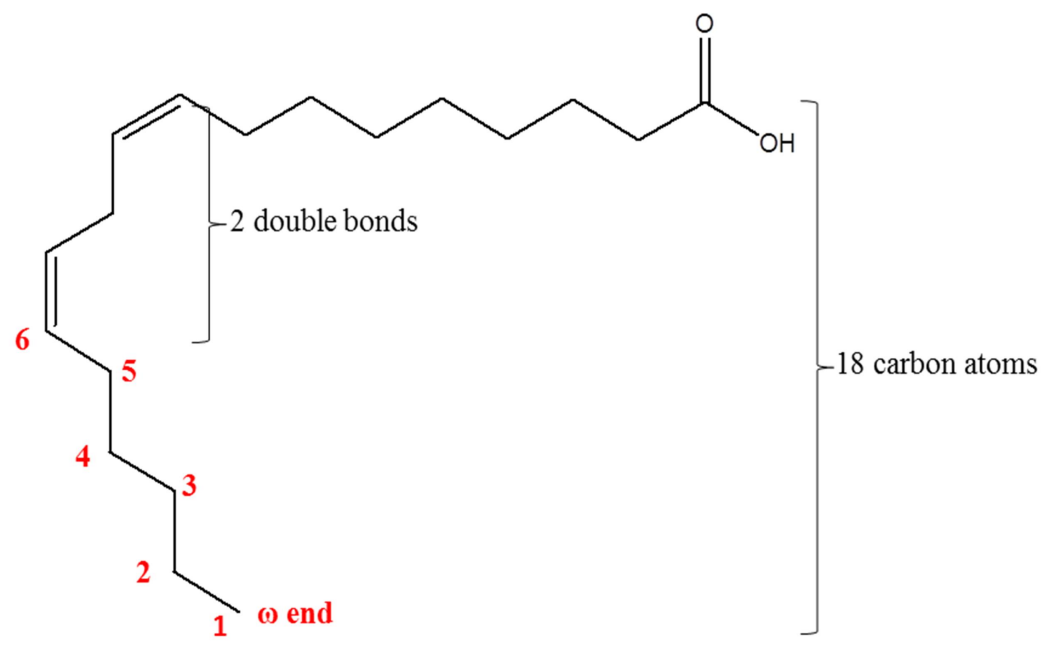

Figure 24. The structure of linoleic acid (LA), short formula 18:2n-6.

For our purposes and based on the extent of unsaturation, fatty acids can be grouped in three categories: saturated, monounsaturated and polyunsaturated. More than 20 different fatty acids are found in foods like fruits, vegetable oils, seeds, nuts, milk, animal fats, and fish oils. Essential fatty acids, such as $\omega-3$ fatty acids (those in which the double bonds are located starting at the third carbon from the methyl end-group), have important cellular functions and are required in the human diet because there is no biochemical pathway capable of producing these molecules in the body [80].

A. Saturated fatty acids (SFA)

Saturated fatty acids (SFA) have only a single carbon-carbon bond; therefore they contain the maximum number of hydrogen atoms attached to every carbon atom. Most 
SFAs are straight hydrocarbon chains with an even number of carbon atoms. The most common fatty acids contain 12-22 carbon atoms. Some particularly representative examples of SFAs are palmitic acid (C16:0), stearic acid (C18:0), and myristic acid (C14:0). Consumption of saturated fatty acids increases low-density lipoprotein cholesterol levels and it has also been associated with an increased risk of coronary heart disease [81, 82]. However, new studies on cellular and physiological functions of individual SFAs emphasizes that short and medium chain SFAs, and myristic acid (C14:0) have important cellular and metabolic roles [83].

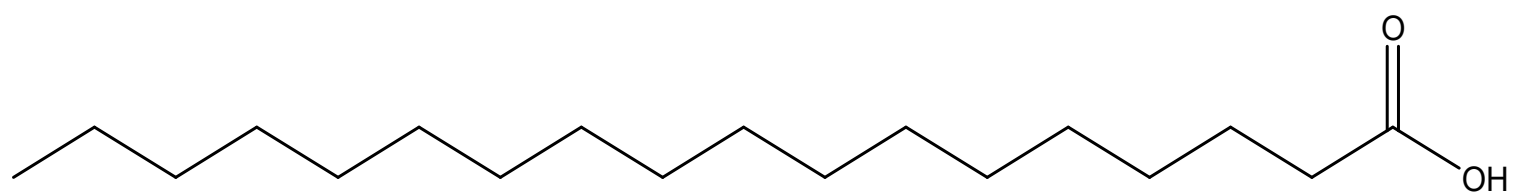

Figure 25. The structure of a saturated fatty acid, stearic acid (18:0).

B. Monounsaturated fatty acids (MUFA)

Monounsaturated fatty acids (MUFA) have one carbon-carbon double bond that can be located in several different positions relative to the methyl-end group. The orientation of the hydrogen atoms around the double bond determines the geometric configuration. In a cis $(\mathrm{Z})$ configuration, the hydrogen atoms are present on the same side of the double bond, while in the trans (E) configuration, they are on opposite sides. The most common naturally occurring MUFAs have a chain length of $16-22$ carbon atoms and a double bond having a cis geometry. Oleic acid (cis C18:1n-9) comprises about $92 \%$ of cis MUFAs in the human diet [84]. The other common MUFAS are cis- 
palmitoleic acid (cis 16:1n-7), and cis-vaccenic acid (cis 18:1n-7). Studies have found that an increased intake of monounsaturated fatty acids correlates with a decreased risk of coronary heart disease $[80,84]$. Geometrical isomerism (cis/trans isomerism) modulates the shape and physical properties of the molecule; cis fatty acids have a 'kink' in the chain whereas trans fatty acids (TFA) are 'straight' and function comparably to SFA [85]. The cis fatty acids are thermodynamically less stable than the trans form. They have lower melting points and are consequently often liquids at room temperature [86].

(a)

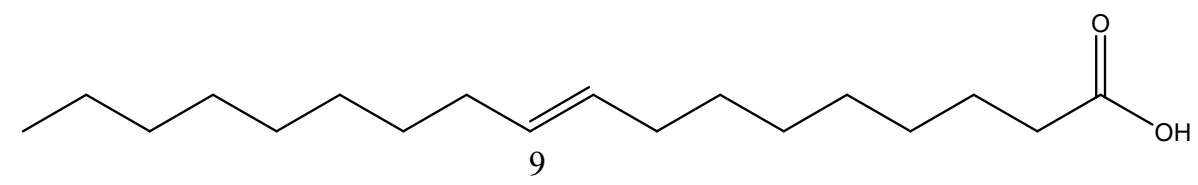

(b)

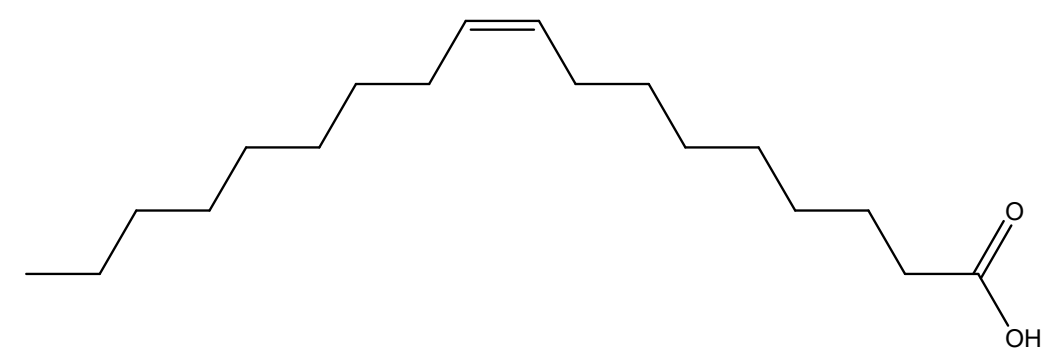

Figure 26. The structures of the monounsaturated fatty acids of oleic acid (18:1n-9) (a) the trans structure, elaidic acid and (b) the naturally occurring cis structure.

C. Polyunsaturated fatty acids (PUFA)

Polyunsaturated fatty acids (PUFA) have two or more carbon-carbon double bonds. If the first double bond is found between the third and the fourth carbon atom from the $\omega$ carbon, these are called $\omega-3$ or $n-3$ fatty acids. If the first double bond is between the sixth and seventh carbon atom, then they are called $\omega-6$ or n-6 fatty acids. 
The double bonds in PUFAs are usually unconjugated and frequently separated from each other by a methylene group [79].

Humans acquire PUFAs from their diet. They are metabolized in the body by elongation (adding carbon atoms) or by further desaturation (extraction of hydrogen atoms). Desaturases are capable of forming new double bonds between carbon atoms found between an existing double bond and the carboxyl group. Linoleic acid (LA or 18:2n-6) and $\alpha$-linolenic acid (ALA or 18:3n-3) are the principal essential PUFAs. LA and ALA are the precursors of arachidonic acid (20:4n-6), eicosapentaenoic acid (EPA; 20:5n-3) and docosahexaenoic acid (DHA; 22:6n-3), respectively. A beneficial effect on the cognitive development of infants and children is attributed to dietary supplementation during pregnancy and lactation with n-3 fatty acids [80].

(a)

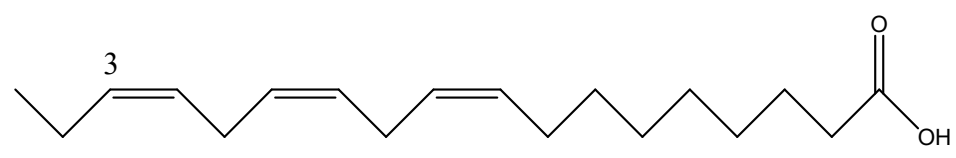

(b)

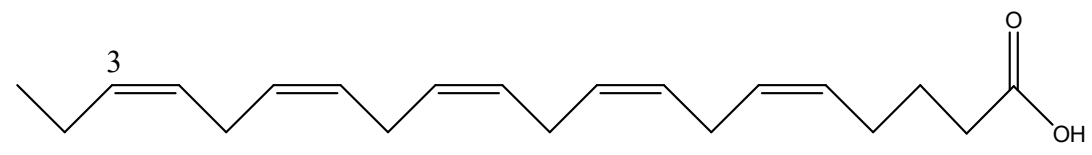

(c)

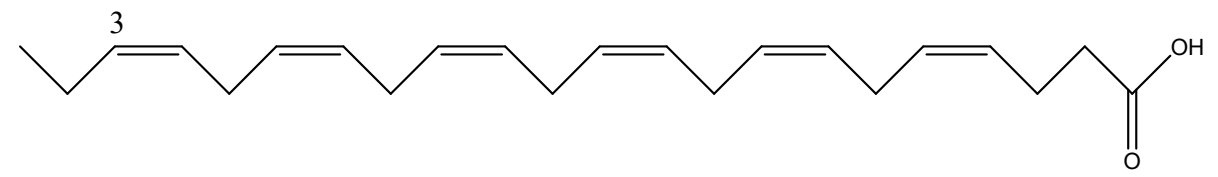

Figure 27. The structures of polyunsaturated fatty acid, (a) $\alpha$-linolenic acid (ALA, 18:3n3), (b) eicosapentaenoic acid (EPA, 20:5n-3), and (c) docosahexaenoic acid (DHA, 22:6n-3). 


\subsection{Lipids in Human Milk}

\subsubsection{Milk fat globules}

Human milk fat globules are the lipid delivery carriers secreted by the mother for nutritional purposes. They provide $40-55 \%$ of the total energy intake for infants [87]. The milk fat globule is formed by a unique mechanism that provides a specific composition and structure [88]. The fat globules in human milk are typically about 1 to $10 \mu \mathrm{m}$ in diameter. Most of them (70-90\%) are about $1 \mu \mathrm{m}$ but most of the lipid mass is contained in those that are about $4 \mu \mathrm{m}$. The large surface area of these globules, particularly the smaller globules, can bind many lipase enzymes that will contribute to triglyceride digestion [89]. The core of the globules is mainly composed of triacylglycerols and nonpolar lipids such as cholesteryl and retinyl esters. The outer surface of fat globules consists of bipolar components such as phospholipids, proteins, mucopolysaccharides, cholesterol, and enzymes [75]. The thin phospholipid layer is commonly referred as the milk fat globule membrane. Milk lipids must be liquid in order to form microdroplets within the secreting cells and they will fuse into droplets that will finally be secreted as globules [75]. Studies have reported that the polar lipids in the milk fat globule membrane have different benefits for human health as a result of their involvement in cell functions such as growth, development, and transport systems [87].

\subsubsection{Origins of milk fat}

Mammary alveolar cells are stimulated to produce milk fat by prolactin secreted from the anterior lobe of the pituitary gland and by emptying of the breast after lactation [89]. Fatty acids in milk can come from the uptake from circulation or from de novo 
synthesis within the mammary epithelial cells. Although many fatty acids are of dietary origin others are formed by metabolism of dietary fatty acids in the maternal liver [90]. Short-chain fatty acids (4 to 8 carbons) and medium-chain fatty acids (10 to 14 carbons) are produced almost exclusively from de novo synthesis [91]. Fatty acids with 16 carbons come from either de novo synthesis or from the uptake, while long-chain fatty acids ( $>16$ carbons) are derived exclusively from the uptake of circulating lipids derived from dietary intake. Preformed fatty acids are taken up from circulating lipoproteins by the mammary gland and may be used as the building blocks for further synthesis of the fats that ultimately comprise milk fat. Non-esterified fatty acids originate from the absorption of lipids from the digestive tract and from the mobilization of body fat reserves [91].

Table 13. Factors associated with changes in the total lipid content of human milk (adapted from [75] p. 1254)

\begin{tabular}{|l|l|}
\hline Factor & Effect \\
\hline Duration of a nursing or feeding & Increase \\
\hline Age postpartum, stage of lactation & Increase \\
\hline Diurnal rhythm & $\begin{array}{l}\text { Variable (related to time of samples and maternal } \\
\text { meals) }\end{array}$ \\
\hline Between breast & Occurs \\
\hline Gestational age at birth (pretermn vs. term) & Occurs \\
\hline Diet regionality & May Occur \\
\hline Nutrition status & Decrease in malnourished women \\
\hline High carbohydrate, low fat diet & May decrease \\
\hline Infections, metabolic disorders & Usually decreases \\
\hline Parity & Decrease \\
\hline Season & Related to diet and regionality \\
\hline Individuality & Adiposity increases \\
\hline
\end{tabular}


The volume of human milk produced by each mother as well as macronutrients and micronutrients varies among individuals. The composition of human milk is influenced by genetics, nutrition, and other factors. In Table 13, a list of factors that have been observed to influence the fat content in human milk and their effects is presented [75]. As seen in Table 13, the lipid content in human milk can be influenced by different factors like diet, duration of nursing, and parity.

\subsubsection{Lipids in human milk}

Triacylglycerols (TG) are the main component of the lipids in milk accounting for more than $98 \%$ of the total fat. Phospholipids and cholesterol contribute about $0.7 \%$ and $0.5 \%$ of milk, respectively $[77,89]$. The TG structure controls the liquidity of the lipid globules and the identity of the products formed by the lipolysis reactions in the stomach and intestine [75]. Table 14 shows data taken from a study [92] of the composition of milk in full- and pre-term mothers. From this Table, we can compare the changes between the first and the $12^{\text {th }}$ week of lactation. As seen in Table 14, the percent total lipid in milk increases with time as well as the amount of triacylglycerols.

Table 14. Lipid class composition during lactation (adapted from [92]). Amounts in wt $\%$, total lipid $\%$ in mean \pm SEM.

\begin{tabular}{|c|cccccc|}
\hline & \multicolumn{5}{|c|}{ Percentage of total lipids at different lactation days } \\
days & $\mathbf{3}$ & $\mathbf{7}$ & $\mathbf{2 1}$ & $\mathbf{4 2}$ & $\mathbf{8 4}$ \\
\hline $\mathbf{n}$ & 39 & 41 & 25 & 18 & 8 \\
\hline Total lipid \% in milk & $2.04 \pm 1.32$ & $2.89 \pm 0.31$ & $3.45 \pm 0.37$ & $3.19 \pm 0.43$ & $4.87 \pm 0.62$ \\
Phospholipid & 1.1 & 0.8 & 0.8 & 0.6 & 0.6 \\
Cholesterol & 1.3 & 0.7 & 0.5 & 0.5 & 0.4 \\
Triacylglycerol & 97.6 & 98.5 & 98.7 & 98.9 & 99.0 \\
Cholesteryl esters (mg/dL) & 5 & 1 & 1 & 1 & 1 \\
\hline
\end{tabular}




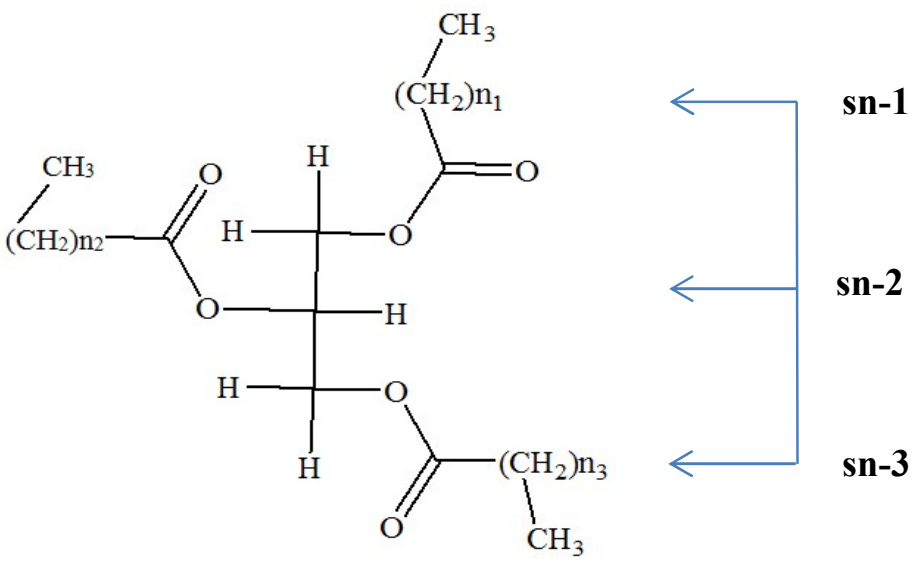

Figure 28. The stereospecific number of the substitution of fatty acids in triacylglycerols.

Human milk usually contains seven major fatty acids in amounts higher than $1 \%$. TG vary in the kinds and amounts of fatty acids present in their structure. In the TG structure, three positions are available for different types of fatty acids (see Figure 15) and the distribution of fatty acids is not random. The stereospecific numbering (sn) designates the location of fatty acids within the triglyceride molecule (see Table 16). The distribution of fatty acids between the outer positions ( 1 and 3, referred as sn-1 and sn-3, see Figure 15) and the inner position, sn-2, in the molecule directs the partition between the free and the 2-monoacyl-sn-glycerol forms. The TG substitution pattern consequently modifies the rate of intestinal fatty acid uptake by the body [93]. Therefore, the pattern of fatty acids substitution in TG and their prevalence are unique variables and in different studies $[93,94]$ it has been shown that palmitic acid is favorably located on the sn-2 position. Presence of palmitic acid in this location improves the absorption of fat and calcium in the small intestine of infants [75]. The other two major fatty acids in human milk, oleic and linoleic acids, are predominantly esterified at the sn- 1 and sn-3 positions 
[94]. Human milk differs from infant formulas in fatty acid composition and their positional distribution. Studies have shown human milk fat to be better absorbed in premature and newborn infants because the TG structure influences the way fatty acids are metabolized and incorporated as nutrients [95].

Milk phospholipids comprise on average about $0.6 \%$ of total fat in milk. The major classes of phospholipids are phosphatidyl choline (28.4\%), phosphatidyl ethanolamine (27.7\%), phosphatidyl serine (8.8\%), phosphatidyl inositol (6.1\%), and sphingomyelin (37.5\%). Phospholipids contribute functionally to maintaining the emulsion of fat globules. Human milk has high cholesterol content and cholesterol is the major milk sterol contributing $90.1 \%$ of the total sterol content, followed by desmosterol (8.6\% of total sterols). The maternal diet has no appreciable effect on milk cholesterol and breastfed infants have a relatively large cholesterol intake, about $25 \mathrm{mg} / \mathrm{kg}$ body weight, (relative to adults). A result of this is that plasma cholesterol values are higher in breastfed than in formula-fed infants [89].

Table 15. Distribution (\%) of essential fatty acids in the glycerol structure in colostrum and mature milk (adapted from [96] p. 270). LA = linoleic acid, ALA $=\alpha$-linoleic acid, $\mathrm{AA}=$ arachidonic acid, DHA $=$ docosahexaenoic acid.

\begin{tabular}{|c|cccc|cccc|}
\hline stereospecific & \multicolumn{4}{|c|}{ Colostrum } & \multicolumn{4}{c|}{ Mature milk } \\
numbering position & LA & ALA & AA & DHA & LA & ALA & AA & DHA \\
& $\%$ & $\%$ & $\%$ & $\%$ & $\%$ & $\%$ & $\%$ & $\%$ \\
sn-1 & 35 & 33 & 10 & 8 & 37 & 29 & 6 & 0 \\
sn-2 & 22 & 18 & 45 & 50 & 21 & 26 & 49 & 67 \\
sn-3 & 43 & 49 & 45 & 42 & 42 & 45 & 45 & 33 \\
\hline
\end{tabular}

In humans, glucose-derived acetyl-CoA is the major source of $\mathrm{C} 2$ units for fatty acid synthesis, and the maternal dietary intake of polyunsaturated fatty acids (PUFA) is a 
major contribution to the amounts of n-6 and n-3 fatty acids secreted into mother's milk [96]. The long-chain saturated fatty acids, cis-monounsaturated fatty acids, trans-fatty acids and n-6 and n-3 PUFA are taken up by the mammary gland from circulation and then acylated into PUFAs for secretion in milk. Mammary gland-specific acyl transferases preferentially esterify 16:0 and 18:0 PUFAs at the center sn-2 position in the formation of TG, rather than at the sn-1 or sn-3 positions, as in other tissues [96].

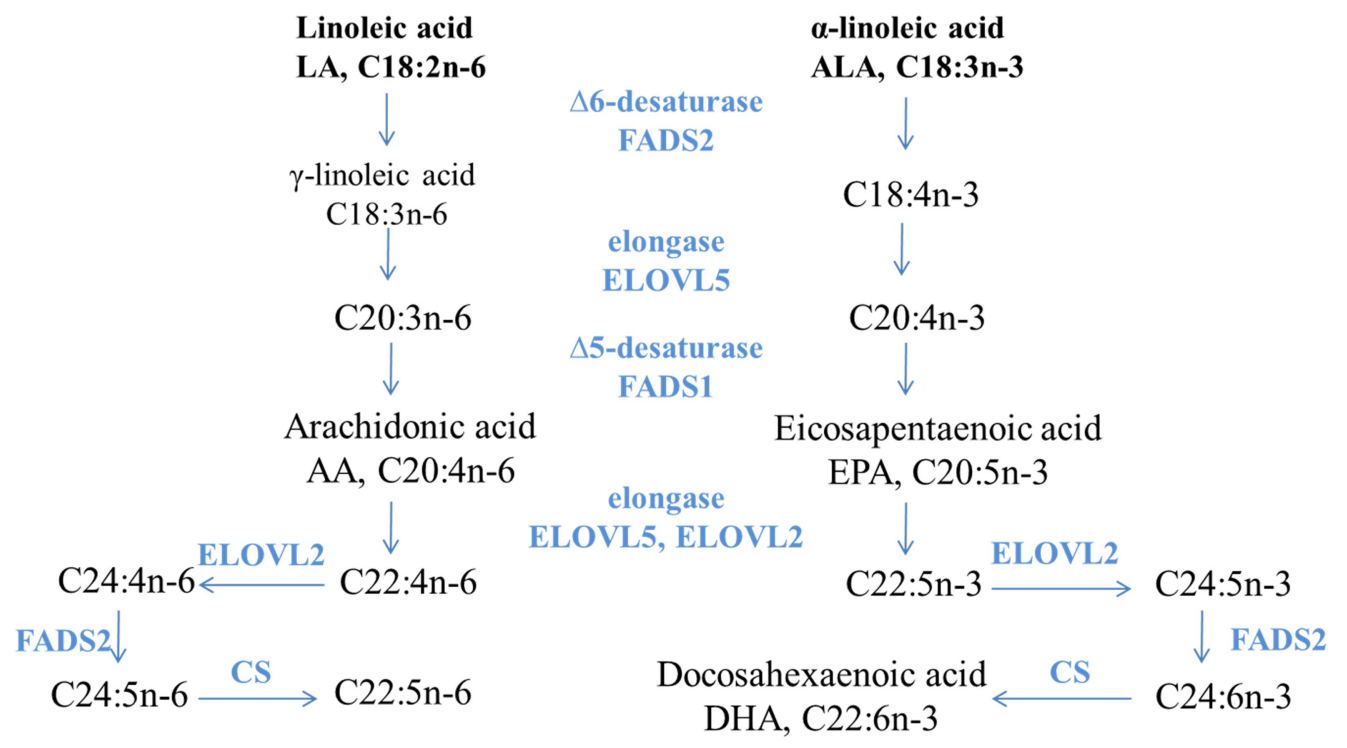

Figure 29. Metabolic conversion of linoleic acid (n-6 series) and $\alpha$-linolenic acid (n-3 series) to form the long-chain polyunsaturated fatty acids (adapted from [97] p. 7).

Humans cannot synthesize essential n-3 and n-6 PUFAs. The parent fatty acids of these families, $\alpha$-linolenic acid (C18:3n-3, ALA) and linoleic acid (C18:2n-6, LA) are essential fatty acids and are required in the daily diet since they cannot be formed by the mammalian cells. ALA and LA are converted to more highly unsaturated fatty acids and to longer chain fatty acids, through enzymatic chain elongation and desaturation. LA and 
ALA are obtained from the diet in high amounts from plant, seed, and nut oils; LA and ALA amounts vary among different types of cooking oils. ALA is sequentially elongated and desatured to eicosapentaenoic acid (C20:5n-3, EPA) and then to docosahexaenoic acid (C22:6n-3, DHA). LA is elongated and desaturated to arachidonic acid (C20:4n-6, AA). DHA is an especially important and major component of cell membranes in the brain and the retina. AA is also a membrane component and it is a precursor to the potent signaling molecules, the prostaglandins and leukotrienes [97].

The pathway for conversion of essential fatty acids to AA and DHA is shown in Figure 29. Both n-3 and n-6 PUFAs are substrates of the same chain elongation/desaturation enzymes, and consequently there is a competition between them for the enzyme [97]. Therefore, the balance between LA and ALA in the diet is very important. The significance of this competition is that an excessive amount of n-6 can reduce the production of DHA and EPA, which is needed for optimal neural development. Moreover, excessive production of AA can lead to imbalances contributing to prostagland and leukotrin production leading to pro-inflammatory conditions [98].

Low rates of DHA synthesis from ALA appear to be a general characteristic of human metabolism, with the slowest step in (n-3) fatty acid desaturation being the conversion of EPA to DHA [99]. It has been observed that dietary DHA is well absorbed and readily incorporated into plasma and blood cell lipids in humans. Animal studies have also shown that dietary DHA is readily incorporated into lipids of the developing brain before and after birth [99]. As mentioned earlier, DHA is particularly important in the central nervous and visual system. In the brain, DHA is enriched in synaptic terminal 
membranes, has diverse roles in brain growth and function, and protects against oxidative stress [99]. High concentrations of DHA are present in phosphatidylserine and the ethanolamine phosphoacylglycerols (ethanolamine plasmalogen and phosphatidylethanolamine) of brain grey matter and the outer segments of rod and cone photoreceptors in the retina DHA comprises $\leq 80 \%$ of the PUFA in the retina outer segment disks. Phospholipids present in both fatty acids are DHA [96]. A study by Niu et al. [100] has shown that low levels of DHA caused by $n$-3 FA deficiency leads to suboptimal function in learning, memory, olfactory-based discrimination, spatial learning, and visual acuity.

TFA are trans isomers of unsaturated fatty acids which can also be found in human milk. TFA originate from the maternal diet because humans cannot synthesize them. They are formed from ruminant fats by hydrogenation and from biohydrogenation of dairy foods. Trans isomers can also be formed from the partial hydrogenation of vegetable oils [101]. Margarine, frying fats, and baking shortenings may contained up to 40-50\% TFA while ruminant fats contain 1-8\% [102]. High intakes of TFA have shown to have a correlation with an increased risk of coronary heart disease, but when the data were evaluated, moderate intakes were not associated with increased risk [103]. As mentioned earlier in this review, a mayor difference of TFA and cis-fatty acids is their melting points. The melting point of oleic acid (cis $\mathrm{C} 18: 1$ ) is $10-11^{\circ} \mathrm{C}$, while the melting point of elaidic acid (trans $\mathrm{C} 18: 1$ ) is $44.5-45.5^{\circ} \mathrm{C}$. The trans isomer is also betterabsorbed and incorporated into tissue lipids than its corresponding cis fatty acids [75]. 


\subsubsection{Review of Previous Studies}

Over the past few decades, studies on the composition of human milk fatty acids in women from different countries around the world have presented the variation in the amounts of fatty acids among diverse populations. In order to better understand this variability we chose to study a population that has not been previously studied, in this case a Peruvian population.

Table 16. List of studies grouped according to their geographic location, country and first author of the citation.

\begin{tabular}{|c|c|c|c|c|c|c|c|}
\hline Europe & $\begin{array}{c}\text { North } \\
\text { America }\end{array}$ & $\begin{array}{c}\text { South } \\
\text { America }\end{array}$ & Africa & Oceania & Asia & South Asia & $\begin{array}{c}\text { Middle } \\
\text { east }\end{array}$ \\
\hline $\begin{array}{c}\text { UK, } \\
\text { Yuhas [104] }\end{array}$ & $\begin{array}{l}\text { Canada, } \\
\text { Yuhas[104] }\end{array}$ & $\begin{array}{c}\text { Chile, } \\
\text { Yuhas[104] }\end{array}$ & $\begin{array}{l}\text { Nigeria } \\
\text { (urban), } \\
\text { Glew[105] }\end{array}$ & $\begin{array}{l}\text { Australia, } \\
\text { Yuhas[104] }\end{array}$ & $\begin{array}{c}\text { China, } \\
\text { Yuhas[104] }\end{array}$ & $\begin{array}{l}\text { Phillipines, } \\
\text { Yuhas[104] }\end{array}$ & $\begin{array}{c}\text { West Iran, } \\
\text { Bahrami } \\
{[106]}\end{array}$ \\
\hline $\begin{array}{c}\text { Sweden, } \\
\text { Xiang[107] }\end{array}$ & $\begin{array}{c}\text { US, } \\
\text { Yuhas[104] }\end{array}$ & $\begin{array}{l}\text { Argentina, } \\
\text { Marin[108] }\end{array}$ & $\begin{array}{c}\text { Nigeria } \\
\text { (rural), } \\
\text { Glew[105] }\end{array}$ & $\begin{array}{c}\text { Australia, } \\
\text { Makrides } \\
{[109]}\end{array}$ & $\begin{array}{c}\text { North China, } \\
\text { Wan[110] }\end{array}$ & $\begin{array}{c}\text { West India, } \\
\text { Roy[111] }\end{array}$ & $\begin{array}{c}\text { Iraq, Al- } \\
\text { Tamer } \\
{[112]}\end{array}$ \\
\hline $\begin{array}{l}\text { Germany, Genzel- } \\
\text { Boroviczeny[113] }\end{array}$ & $\begin{array}{c}\text { Mexico, } \\
\text { Yuhas[104] }\end{array}$ & $\begin{array}{c}\text { Bolivia, } \\
\text { Martin[114] }\end{array}$ & $\begin{array}{c}\text { Mauritius, } \\
\text { Pugo- } \\
\text { Gunsan[115] }\end{array}$ & & $\begin{array}{c}\text { Japan, } \\
\text { Yuhas[104] }\end{array}$ & $\begin{array}{c}\text { Nepal, } \\
\text { Schmeits } \\
{[116]}\end{array}$ & $\begin{array}{c}\text { Israel, } \\
\text { Saphier } \\
\text { [117] }\end{array}$ \\
\hline $\begin{array}{l}\text { Spain, Lopez- } \\
\text { Lopez[94] }\end{array}$ & & $\begin{array}{c}\text { Brazil, } \\
\text { Nishimura } \\
\text { [118] }\end{array}$ & & & & & $\begin{array}{c}\text { Turkey, } \\
\text { Aydin } \\
\text { [119] }\end{array}$ \\
\hline $\begin{array}{c}\text { Portugal, } \\
\text { Ribeiro[120] }\end{array}$ & & & & & & & \\
\hline Italy, Serra[121] & & & & & & & \\
\hline $\begin{array}{c}\text { France, Pugo- } \\
\text { Gunsan[115] }\end{array}$ & & & & & & & \\
\hline $\begin{array}{c}\text { Croatia, } \\
\text { Kresic[122] }\end{array}$ & & & & & & & \\
\hline Serbia, Arsic[123] & & & & & & & \\
\hline $\begin{array}{l}\text { Hungary, } \\
\text { Decsi[124] }\end{array}$ & & & & & & & \\
\hline
\end{tabular}

The following paragraphs presents a summary of previous studies that include data from 29 different countries also compares populations in various geographic regions. The stage of lactation for the sample collection in these studies varies from day 14 up to 12 months post-partum. We have chosen to collect and compare information from 
studies with mature milk and those from a full-term pregnancy. The size of the sample populations in each study ranged from 13 subjects to a maximum of 83 subjects, with an average of 39 subjects per study. The preferred method for analyzing the fatty acids was as methyl esters by gas chromatography, although one study using derivatization by 2nitrophenylhydrazide and analysis by HPLC is also included. Table 17 shows the distribution of the countries by region. The countries studied are ordered into eight regions based on their geographic locations. Refer to Appendices 1 to 5 for a summary of the contents of the most abundant FAs in maternal milk in these 29 countries.

Palmitic acid is the major saturated fatty acid in human milk. Italy, Sweden, France, and Nigeria have the highest levels $(25.00-29.26 \%)$ of this saturated fatty acid, while Chile, Mauritius, China and Canada $(18.50-18.80 \%)$ have the lowest. Lauric acid (C12:0), myristic acid (C14:0), and stearic acid (C18:0) are the next most abundant SFA present in human milk. The mean amounts found for each of them are $6.87 \%, 8.28 \%$, and 5.95\%, respectively. Nigeria, Phillipines and Iraq show higher amounts of lauric and myristic acids in milk, which range up to 23 weight $\%$ a value almost double the median for the rest of the countries' data collected in this review. This tendency (toward high levels of medium length FA) has been shown to be common in countries with a highcarbohydrate and low fat intake diet, which leads to a high percentage of de novo synthesis from glucose in the mammary gland [125]. 

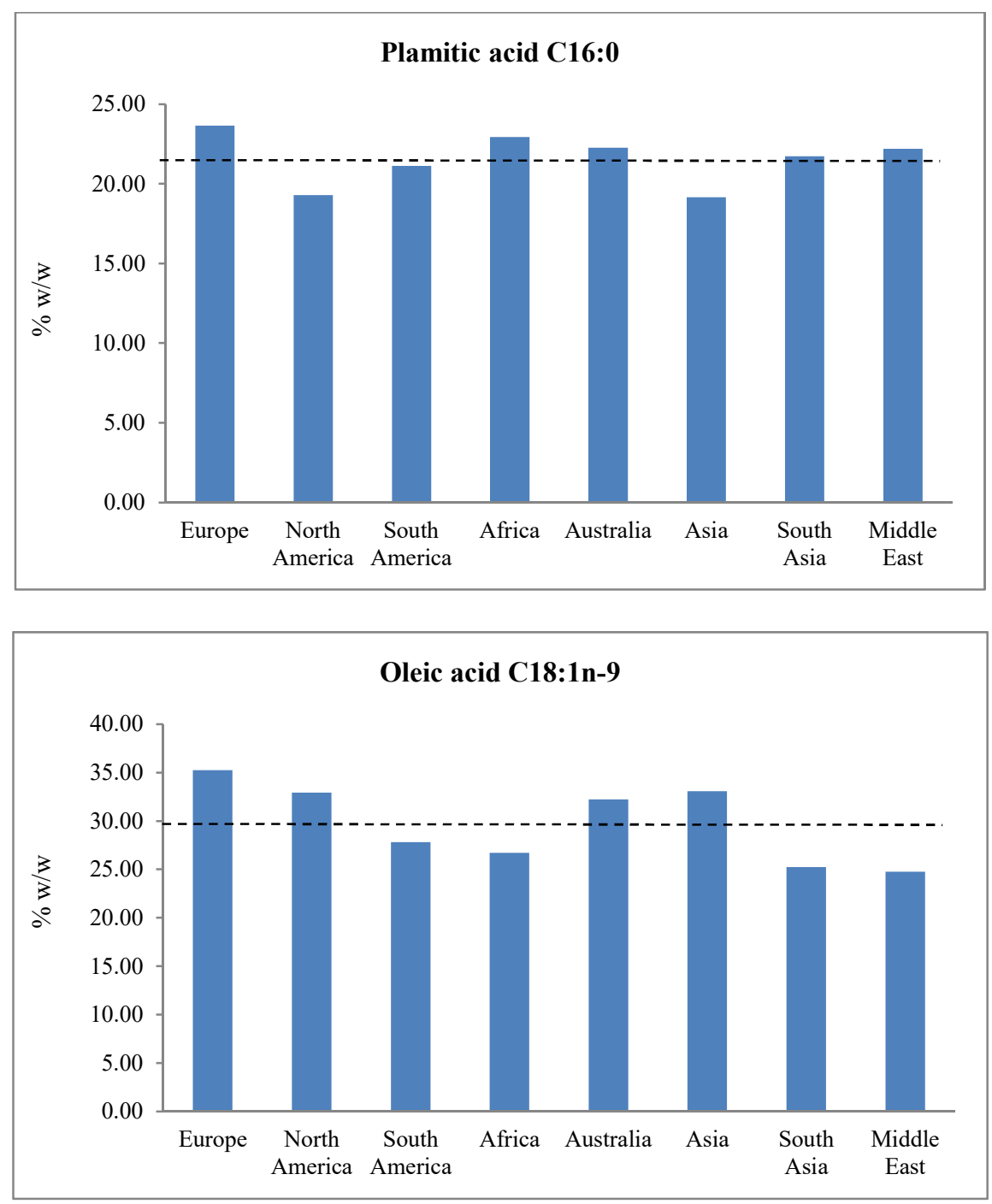

Figure 30. Fatty acids concentrations in human milk summarized by geographic regions of the world a) SFA: palmitic acid, mean $=21.5 \% \mathrm{w} / \mathrm{w}$; b) MUFA: oleic acid, mean $=29.8 \%$. w/w. (-----) represents the mean amount.

MUFAs are higher in the European, North Asian, and North American regions. Oleic acid is the major MUFA in human milk and it is also the most abundant of all the fatty acids present. The average amount of oleic acid across all countries was 29.8 weight $\%$ (see Figure 29). The Oleic acid content in milk is higher than average in most of the highly-developed countries including Canada, US, Australia, and among many countries in Europe. Oleic acid levels in human milk are produced by de novo synthesis 
as well as from dietary intake. Consumption of animal fat and vegetable oils like olive and canola oil increases the concentrations of oleic acid in human milk.
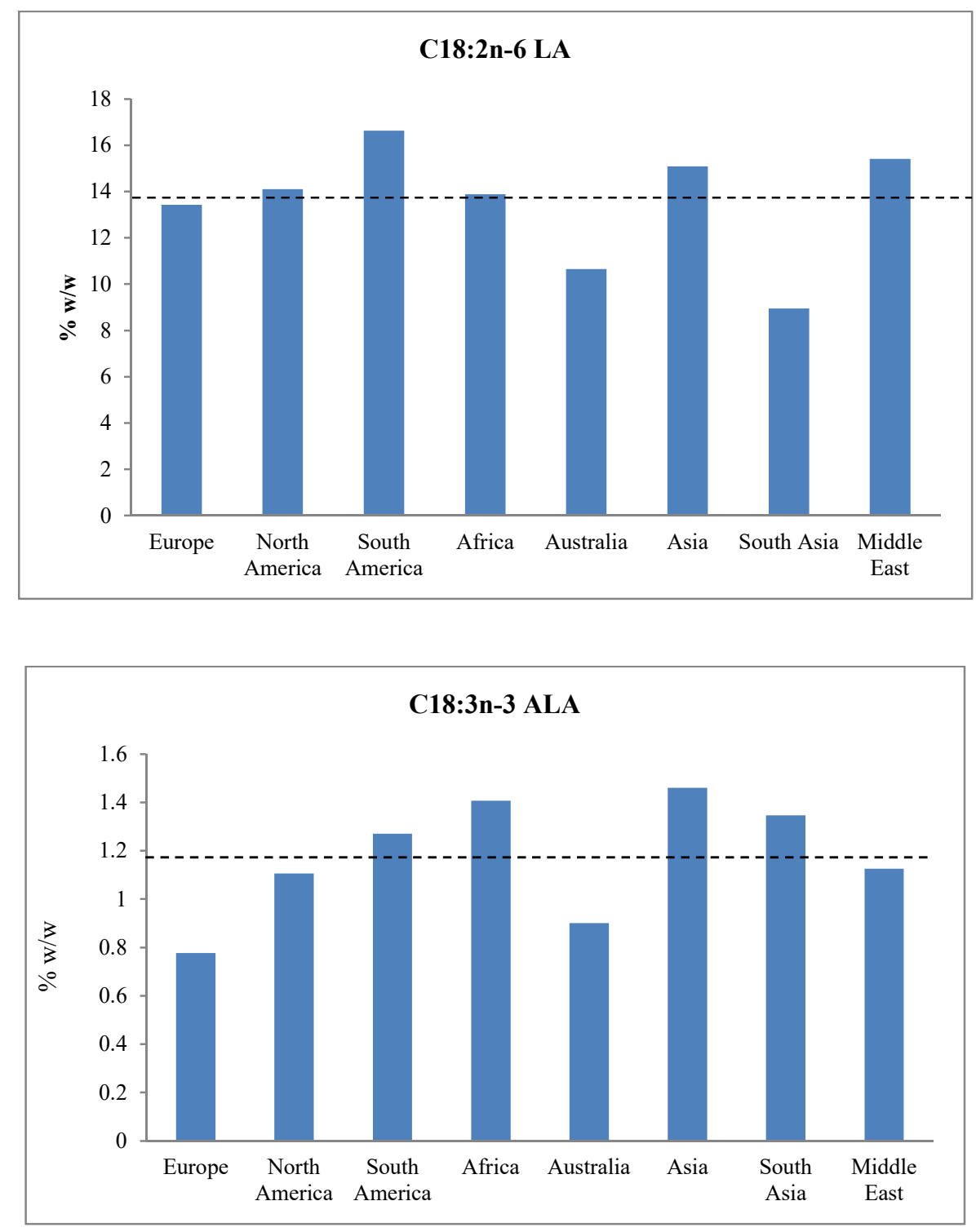

Figure 31. Essential PUFAs summarized by geographic regions over the world A) Linoleic acid, mean $=13.5 \% \mathrm{w} / \mathrm{w}$. B) $\alpha$-linoleic acid, mean $=1.2 \% \mathrm{w} / \mathrm{w} .-(---\quad$ ) represents the mean amount.

Human milk provides the essential PUFAs (LA, ALA, DHA, AA, and other long chain PUFAs) to breastfed infants. These FA in human milk are important for the new 
born for different reasons: 1) they are a major energy source, 2) they are necessary to support appropriate cell growth, and 3) they are required for normal growth and maturation of critical organ systems, particularly the brain and the eyes.

The amounts of essential fatty acids, LA (C18:2n-6) and ALA (C18:3n-3), in human milk are determined by the maternal diet. Mauritius has the highest amount of LA among all the countries $(26.85 \% \mathrm{w} / \mathrm{w})$. South American countries have elevated levels of LA. This is understandable in countries such Brazil and Argentina, where consumption of red meat is traditionally higher than for other populations. Another contribution to the high levels of LA in countries such as Chile and Mexico is the frequent intake of corn. Some countries in the Middle East such as Israel also showed a high amount of LA (20.00\%). On the other hand, Nepal, Western India, and Nigeria have the lowest amounts of LA $(\sim 7-7.91 \% \mathrm{w} / \mathrm{w})$.

ALA (n-3) can be obtained in the diet through intake of vegetable oils and nuts (for example flax seed oil, soy bean oil, and walnuts). The amounts of ALA present in human milk are on average approximately ten times lower than those of LA according to our review of the literature (see Figure 30).

AA, EPA and DHA found in human milk may originate from the maternal diet or from synthesis from LA and ALA in the maternal tissues [96]. The amounts of AA in human milk found in our review range between $0.16-1.4 \% \mathrm{w} / \mathrm{w}$ of total fatty acids, and the amount of DHA range from $0.12-0.99 \% \mathrm{w} / \mathrm{w}$ of total fatty acids. In the publication by Brenna et al. in 2007 [126], it was stated that the variation of the levels of AA are lower than those of DHA in mature milk. Reviewing data collected from most recent and geographically diverse populations leads us to disagree with the conclusion. 

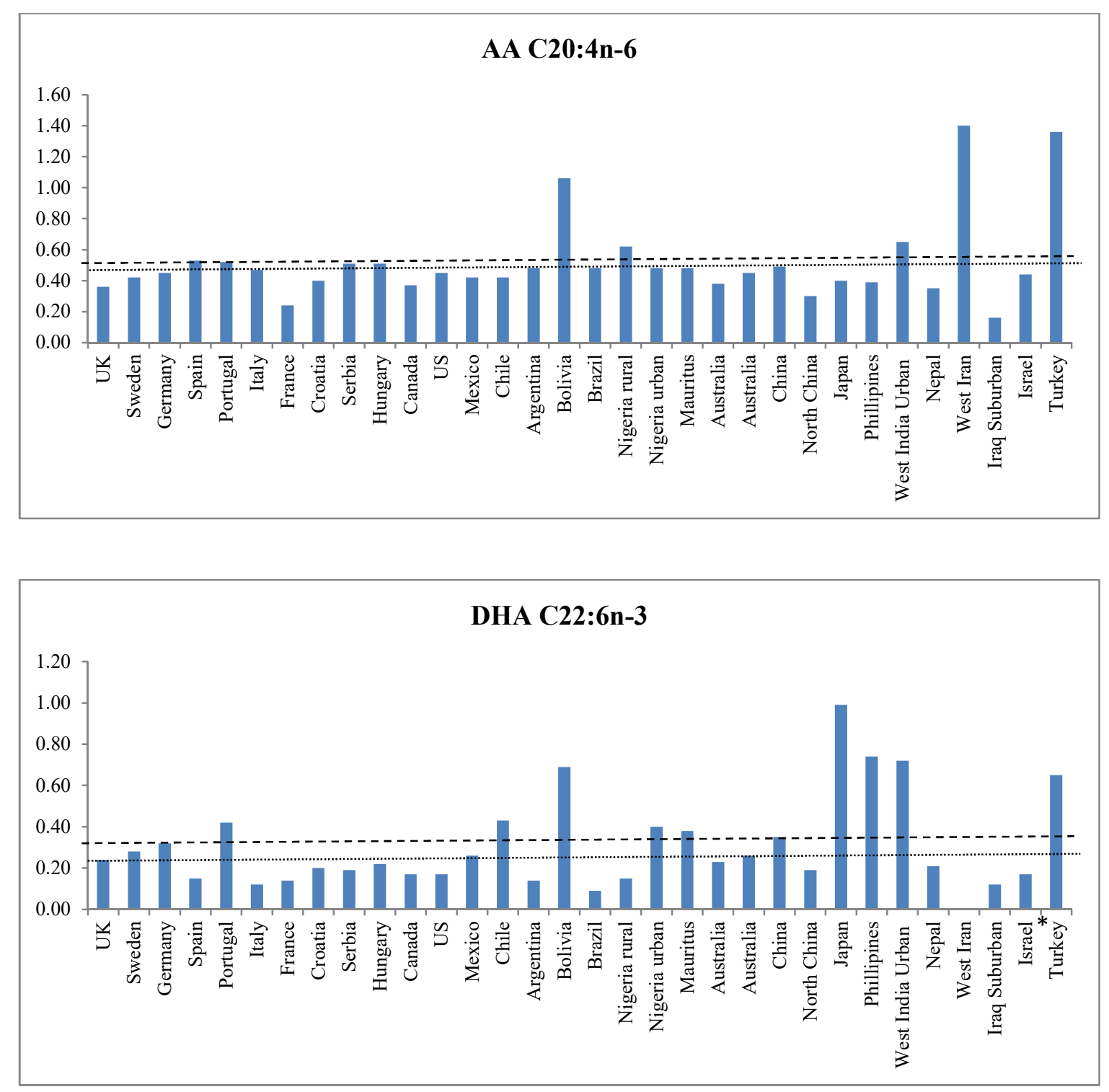

Figure 32. Long Chain-PUFAs by country. A) AA, mean $=0.51 \% \quad \mathrm{w} / \mathrm{w}$ and median $=0.46 \% \mathrm{w} / \mathrm{w}$. B) DHA, mean $=0.32 \% \mathrm{w} / \mathrm{w}$ and median $=0.23 \% \mathrm{w} / \mathrm{w}$.

* no data reported. (-----) represents the mean amount and ( ..........) represents the median.

We determined the mean calculated amount of AA found in milk to be 0.51 weight $\%$ (compared with $0.47 \%$ reported by Brenna et al.[126]), and the median was 0.46 weight $\%$. The mean value calculated for DHA content in human milk was 0.32 
weight $\%$. This was consistent with the report mentioned above, and the median was 0.23 weight \% [126]. Studies agreed that the differences of the levels in DHA in human milk from women living in different regions depend on maternal diet [97, 104].

Since all the data reported here were not normalized in any statistical manner for this review, we have presented the unweighted median and the means of the values. Although we have included many countries, not all regions of each country can be sampled because of the nature of each study. There have been more studies conducted in the developed countries, for example those in Europe, than in the less developed countries. By contrast, in Africa data were available for only two countries. In assessing the levels of DHA, we have relied on the review by Brenna et al. that includes 84 publications on DHA to augment data we have been able to independently collect and review.

It has been observed that most of the AA in human milk does not arise from dietary LA conversion but instead can be traced to maternal stores [127]. The biosynthesis of DHA and AA is slow; consequently, higher amounts of DHA in serum during gestation and lactation can be best achieved by consumption of food rich in this PUFA or by supplementation, as has been suggested by different studies [128, 129].

The highest amounts of DHA were found in the maternal milk of Japanese, Philippine, Bolivian, and West Bengalese (in India) women with amounts $\geq 0.69$ weight \%. All of these populations have a high intake of fish. Japan and Philippines are island countries, while West Bengal ranks among the top rural areas in India for fish consumption. The Bolivian population studied was located in the region of the Amazoni 
where consumption of river fish is high. By comparison, the US and Canada have a low average for DHA in maternal milk.

From this brief review we can conclude that the FA composition of human milk is positively affected by the mother's diet and varies with geographic location. Certainly all of the FAs are influenced by the dietary intake, but it evident that the most variable FA among these populations are the medium-chain SFA and DHA.

In the following section, we describe our own results for the analysis of the fatty acid composition of human milk in a Peruvian population. We have compared the FAs' longitudinal variation during a three week period for the first month post-partum. The amounts of 29 FAs are reported. Discussion focuses on the levels of LA, ALA, and DHA since they have such an important role for the healthy development of new born infants. The profiles found in our data are compared with those from other studies that were the topic of the preceding review. We have included a brief qualitative description comparing the principal FA with results from a food questionnaire.

\subsection{Research Methods:}

\subsubsection{Study population}

The participants in this study were 74 Peruvian mothers having a mean age of 26.3 years and BMI of $28.8 \pm 4$ (information available for 69 participants). All participants were in their first pregnancy and had no chronic health concerns. All of the

deliveries occurred after the full 37 weeks of gestation. All volunteers were informed about the study and signed an IRB consent document. 


\subsubsection{Sample collection}

Milk samples from 25 volunteers were collected at three time periods during lactation: the second, third, and fourth weeks after birth. Milk samples from 50 volunteers were collected only during the fourth week after birth. All samples were collected using electric Medela Lactina Select breast pumps, between 9 am and $12 \mathrm{pm}$. All the collections were done at the volunteers' home by appointment. Mothers expressed milk for 10 minutes from a breast that had not been suckled for two hours. After gentle mixing, $20 \mathrm{ml}$ of milk were placed in polypropylene vials. The samples were stored at $-20^{\circ} \mathrm{C}$ for approximately one week at the study site. Samples were transferred weekly to storage at $-80^{\circ} \mathrm{C}$ until they were shipped overnight to our laboratory at Florida International University. Samples were shipped frozen in dried ice.

\subsubsection{Food frequency questionnaire}

All participants were asked about their food consumption practices using a short questionnaire prepared by our nutritionist colleague in the field and based on the usual food intake habits in the area. Diet records were collected during the appointment while milk was being expressed. The data about the mothers' dietary intake were collected based on food frequency intake during a month. Portion size was not assessed.

\subsubsection{Chemicals and instruments}

Methanol (anhydrous purity $\geq 99.8 \%$ ), toluene (HPLC purity $\geq 99.9 \%$ ), acetyl chloride (purity $\geq 99.0 \%$ ), and anhydrous potassium carbonate $\left(\mathrm{K}_{2} \mathrm{CO}_{3}\right)$ were used as solvents and reagents. GC standards include: methyl tridecanoate (C13), Supelco 37 Component fatty acid methyl ester (FAME) mix, methyl laurate (C12), methyl palmitate 
(C16), methyl oleate (C18), and methyl arachidonate (C20). All solvents, reagents and standards were purchased from Sigma-Aldrich.

The standard solution mix, Supelco, contained the following FAMEs components: cis-13,16-Docosadienoic acid methyl ester 2 wt. \%, cis-4,7,10,13,16,19-Docosahexaenoic acid methyl ester 2 wt. \%, cis-11,14-Eicosadienoic acid methyl ester 2 wt. \%, cis5,8,11,14,17-Eicosapentaenoic acid methyl ester 2 wt. \%, cis-8,11,14-Eicosatrienoic acid methyl ester 2 wt. \%, cis-11,14,17-Eicosatrienoic acid methyl ester 2 wt. \%, cis-11Eicosenoic acid methyl ester 2 wt. \%, Methyl cis-10-heptadecenoate 2 wt. \%, Methyl hexanoate 4 wt. $\%$, Methyl $\gamma$-linolenate 2 wt. \%, Methyl arachidate 4 wt. $\%$, Methyl arachidonate 2 wt. \%, Methyl behenate 4 wt. \%, Methyl butyrate 4 wt. \%, Methyl decanoate 4 wt. $\%$, Methyl dodecanoate 4 wt. \%, Methyl elaidate 2 wt. $\%$, Methyl erucate 2 wt. \%, Methyl heneicosanoate 2 wt. \%,Methyl heptadecanoate 2 wt. \%, Methyl linoleate 2 wt. \%, Methyl linolelaidate 2 wt. \%,Methyl linolenate 2 wt. \%, Methyl myristate 4 wt. \%, Methyl myristoleate 2 wt. \%Methyl oleate 4 wt. \%, Methyl octanoate 4 wt. $\%$, Methyl palmitate 6 wt. \%, Methyl palmitoleate 2 wt. \%, Methyl pentadecanoate 2 wt. $\%$, Methyl cis-10-pentadecenoate 2 wt. \%, Methyl stearate 4 wt. \%, Methyl tricosanoate 2 wt. \%, Methyl tetracosanoate 4 wt. \%, Methyl tridecanoate 2 wt. \%, Methyl undecanoate 2 wt. \%, and Methyl cis-15-tetracosenoate 2 wt. $\%$.

The analyses were carried-outusing a Hewlett Packard 6890 gas chromatograph equipped with a flame ionization detector (FID). FAME was separated on a silica column (30 m x $0.20 \mathrm{~mm}$ ID) coated with SP-2330 stationary phase poly $(80 \%$ biscianopropyl-20\% cyanopropylphenylsiloxane), having a $0.20 \mu \mathrm{m}$ film thickness. 


\subsubsection{Determination of FAME in human milk}

Fatty acids were converted to methyl esters following the method used by LopezLopez and co-workers [130]. $100 \mu \mathrm{L}$ of human milk sample was placed in a 16 x $125 \mathrm{~mm}$ screw Teflon cap Pyrex tube with a magnetic stirring bar, together with $40 \mu \mathrm{L}$ of a

toluene solution containing $3 \mu \mathrm{g} / \mu \mathrm{L}$ tridecanoic acid methyl ester, and $2 \mathrm{~mL}$ of a $4: 1(v / v)$ solvent mixture of methanol/toluene. While stirring, $200 \mu \mathrm{L}$ of acetyl chloride was slowly added and then the tube was sealed. The tube was transferred to a $100^{\circ} \mathrm{C}$ water bath and allowed to react for 1 hour. After reaction, the tube was transferred to an ice bath to stop the reaction and $5 \mathrm{~mL}$ of $6 \% \mathrm{~K}_{2} \mathrm{CO}_{3}$ solution was added to neutralize the solution. The tube was then vortexed followed by centrifugation ( $3000 \mathrm{~g}$ ) for 10 minutes. The toluene top layer was transferred to a $2 \mathrm{~mL}$ glass vial, closed with a screw cap and stored at $-20^{\circ} \mathrm{C}$ prior to analysis by GC.

\subsubsection{Gas Chromatography conditions}

The sample, $1 \mu \mathrm{L}$, was manually injected using a syringe in a splitless injector mode. The injector and detector temperatures were $250^{\circ} \mathrm{C}$ and $270^{\circ} \mathrm{C}$, respectively. The oven temperature was programmed as follows: initial temperature $130^{\circ} \mathrm{C}$ for 3 minutes, increasing by $7^{\circ} \mathrm{C} / \min$ to $180^{\circ} \mathrm{C}$, then increasing by $6^{\circ} \mathrm{C} /$ min to a final temperature of $240^{\circ} \mathrm{C}$ for 5 minutes. Helium was used as the carrier gas, with a linear velocity of 22.5 $\mathrm{cm} / \mathrm{s}$, and nitrogen was the make-up gas.

\subsubsection{Identification and quantification of fatty acids}

The standard Supelco FAME mix solution was used to identify the fatty acids. Confirmation of the peaks was accomplished by gas chromatography/mass spectrometry. 
Retention times were determined using the Standard FAME mix solution and C13 was used as an internal standard. Calculation of the amounts of fatty acids was based on peak areas relative to the internal standard.

\subsubsection{Calculations}

The response factor $(R i)$ was determined [131] for each fatty acid in the standard mix solution by using the peak areas and the amounts of FAME in the mixture, using the following formula:

$$
R i=\frac{(A i)(W C 13)}{(A C 13)(W i)}
$$

where $R i$ is the response factor for the FAME $i, A i$ is the peak area of the individual FAME in the standard mix solution, $W C 13$ is the amount of $\mathrm{C} 13$ in the mix solution, and $W i$ is the amount of the $i^{\text {th }}$ FAME in the standard mix solution. The amount of each fatty acid was calculated using the following equation:

$$
Q i=\frac{(A p i)(120)}{(A p C 13)(R i)} \mu g
$$

Eq. 2

where $Q i$ is the amount of individual FAME component $i$ in the sample, Api is the peak area of this individual fatty acid in the sample, $A p C 13$ is the area peak of the internal standard, $\mathrm{C} 13$, added to the sample, and $R i$ is the response factor for the individual FAME from equation 1. All the results obtained from the samples were converted to percentage and expressed as \% of total fatty acids. 


\subsubsection{Partial validation protocol}

For method validation the linearity, limit of detection (LOD), limit of quantification (LOQ), and precision (repeatability) were calculated following the methodology for parameter and test validation using "Validation of analytical methods" by Ludwig Huber [132].

Table 17. Amounts of fatty acid methyl esters $(\mu \mathrm{g})$ used to prepare calibration curves relative to the internal standard.

\begin{tabular}{|l|c|c|c|c|c|}
\hline & $\begin{array}{l}\text { Standard } \\
\text { Solution 1 }\end{array}$ & $\begin{array}{l}\text { Standard } \\
\text { Solution 2 }\end{array}$ & $\begin{array}{l}\text { Standard } \\
\text { Solution 3 }\end{array}$ & $\begin{array}{l}\text { Standard } \\
\text { Solution 4 }\end{array}$ & $\begin{array}{l}\text { Standard } \\
\text { Solution 5 }\end{array}$ \\
\hline $\mathbf{C 1 2}$ & 43.50 & 266.75 & 666.25 & 1132.50 & 1998.75 \\
\hline $\mathbf{C 1 6}$ & 60.00 & 630.00 & 1759.75 & 2495.00 & 3939.50 \\
\hline $\mathbf{C 1 8}$ & 131.00 & 971.00 & 2913.00 & 4855.00 & 7282.50 \\
\hline $\mathbf{C 2 0}$ & 3.75 & 10.00 & 62.50 & 132.50 & 250.00 \\
\hline
\end{tabular}

To check for linearity, calibration curves for the FAMEs C12, C16, C18, and C20 relative to the internal standard (C13) were plotted using five different, increasing concentrations prepared in triplicate for each standard. The amount of $\mathrm{C} 13$ was kept constant for all the solutions at $120 \mu \mathrm{g}$. The increasing amounts for each standard are shown in Table 18. A calibration curve for the internal standard C13 was plotted, using six different concentrations $(0.02,0.1,0.25,0.75,1.5$, and $3 \mu \mathrm{g} / \mu \mathrm{L})$ run in triplicate, versus their peak area count. LOD was calculated using the standard error (STEYX) of the predicted yvalue for each $\mathrm{x}$-value in the regression line, the slope of the regression line, and using Equation 3 [133]: 


$$
L O D=\frac{S T E Y X}{\text { Slope }} * 3
$$

The LOQ was determined as the lowest concentration that we validated in terms of precision and was calculated as ten times the LOD.

In order to check for recovery in the matrix, aliquots of four solutions containing increasing amounts (see standard solutions 2 to 5 shown in Table 18) of C12, C16, C18, and C20 were added to a same milk sample that was randomly selected from the group of collected samples. Each sample was analyzed in 4 replicates. The internal standard was added to each solution and kept at a constant amount of $120 \mu \mathrm{g}$ in all four solutions. The precision was determined by calculating the repeatability of the method. The same sample was analyzed six times during the same day. For reproducibility of the method, the same sample was analyzed on four different days and six different times during each day.

\subsubsection{Statistical Analysis}

Data were analyzed using Statistical Packages for Social Sciences version 21.0 (SPSS Inc. Chicago, IL, USA). For comparison a two-way ANOVA test was conducted for a group of 26 subjects who donated milk samples during the three consecutive weeks of the study.

\subsection{Results}

The method of Lopez-Lopez [130] was used here to measure FAs. The method's experimental conditions were adapted to our instrument and tested for partial validation. 


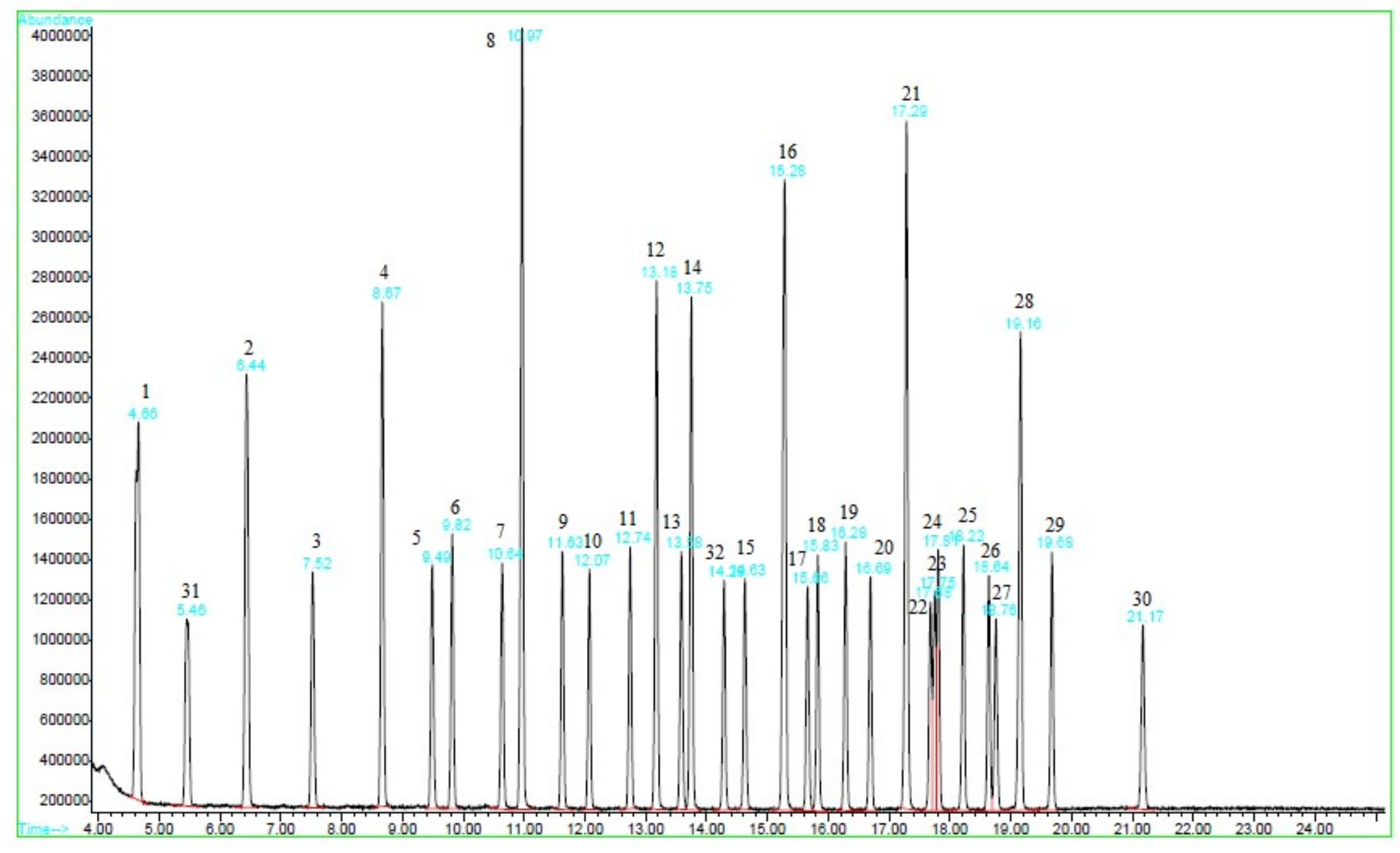

Figure 33. GC-MS of FAME standards. 


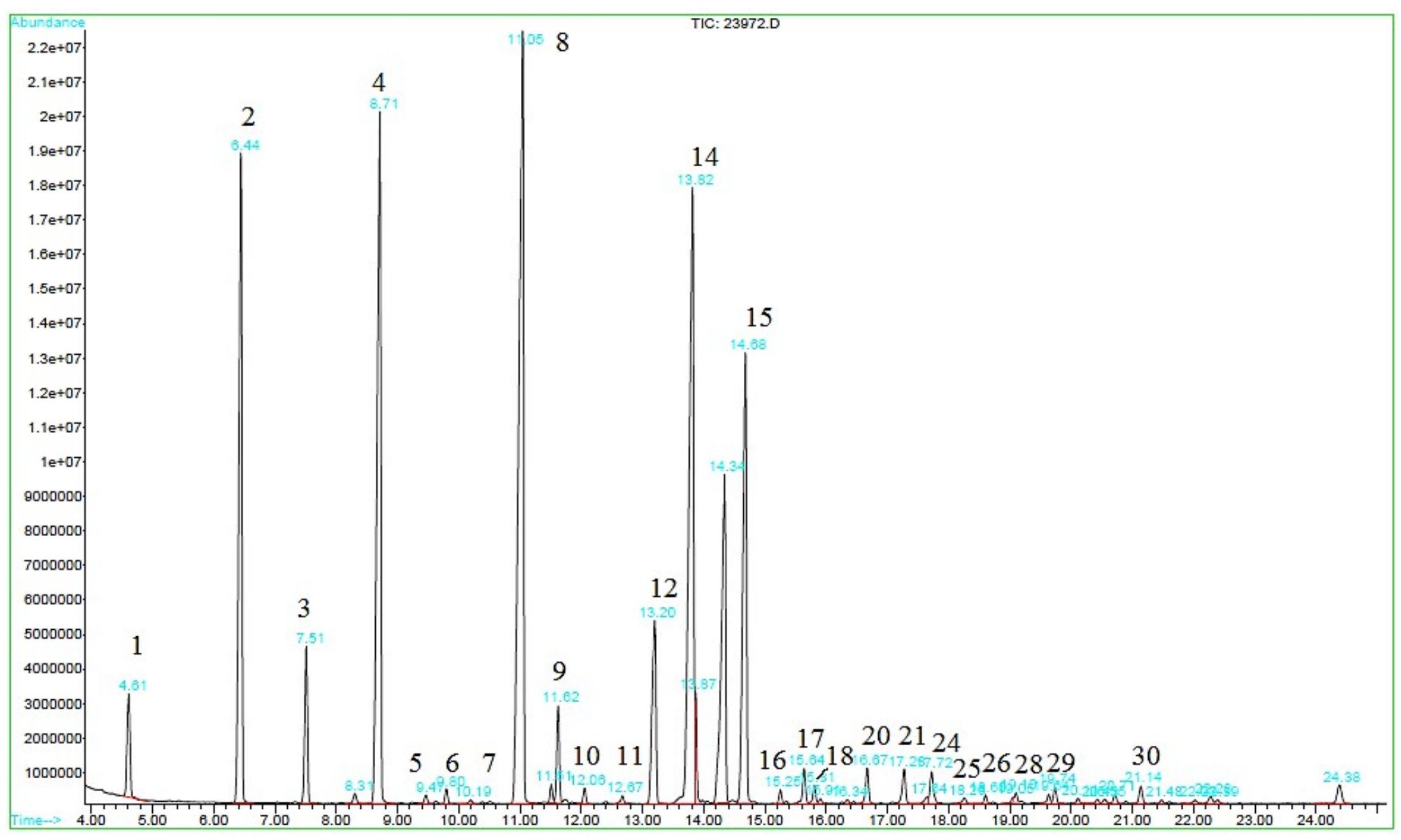

Figure 34. Example of a GC-MS chromatogram of a maternal milk sample. 
Table 18. Retention times and response factors for FAMES calculated from the standard mixture run by GC-FID.

\begin{tabular}{|c|c|c|c|c|}
\hline & Fatty acids & Retention time (minutes) & Weight $(\%$ w/w) & $\mathbf{R i}$ \\
\hline 1 & C10:0 & 5.08 & 4 & 0.82 \\
\hline 2 & C12:0 & 6.97 & 4 & 0.94 \\
\hline 3 & C13:0 internal standard & 8.08 & 2 & 1.00 \\
\hline 4 & C14:0 & 9.26 & 4 & 1.11 \\
\hline 5 & C14:1n-5 & 10.11 & 2 & 1.10 \\
\hline 6 & C15:0 & 10.43 & 2 & 1.19 \\
\hline 7 & C15:1n-5 & 11.28 & 2 & 1.14 \\
\hline 8 & C16:0 & 11.63 & 6 & 1.20 \\
\hline 9 & C16:1n-7 & 12.30 & 2 & 1.18 \\
\hline 10 & C17:0 & 12.75 & 2 & 0.99 \\
\hline 11 & C17:1n-7 & 13.45 & 2 & 1.17 \\
\hline 12 & C18:0 & 13.88 & 4 & 1.04 \\
\hline 13 & C18:1t9 & 14.30 & 2 & 1.07 \\
\hline 14 & C18:1n-9 & 14.47 & 4 & 1.10 \\
\hline 15 & C18:2n-6 & 15.41 & 2 & 1.08 \\
\hline 16 & C20:0 & 16.02 & 4 & 0.93 \\
\hline 17 & C18:3n-3 & 16.43 & 2 & 1.07 \\
\hline 18 & C20:1n-9 & 16.59 & 2 & 0.88 \\
\hline 19 & C21:0 & 17.14 & 2 & 0.76 \\
\hline 20 & C20:2n-6 & 17.47 & 2 & 0.87 \\
\hline 21 & C22:0 & 17.98 & 4 & 0.64 \\
\hline 22 & C20:3n-3 & 18.06 & 2 & 0.83 \\
\hline 23 & C20:3n-6 & 18.47 & 2 & 0.73 \\
\hline 24 & C20:4n-6 & 18.59 & 2 & 0.81 \\
\hline 25 & C23:0 & 19.09 & 2 & 0.59 \\
\hline 26 & C22:2n-6 & 19.45 & 2 & 0.67 \\
\hline 27 & C20:5n-3 & 19.61 & 2 & 0.83 \\
\hline 28 & C24:0 & 19.97 & 4 & 0.54 \\
\hline 29 & C24:1n-9 & 20.52 & 2 & 0.56 \\
\hline 30 & C22:6n-3 & 22.21 & 2 & 0.67 \\
\hline
\end{tabular}


Identification and quantification of FAMEs was carried using a commercially available standard mixture solution. We were able to identify 32 fatty acid methyl esters from the standard mixture (Figure 34) using the relative retention times. All the FAs shown in Table 18 were identified in human milk, in Figure 15 FAs: 13,19,22,23, and 27 are in low amounts (they could not be label in the figure).

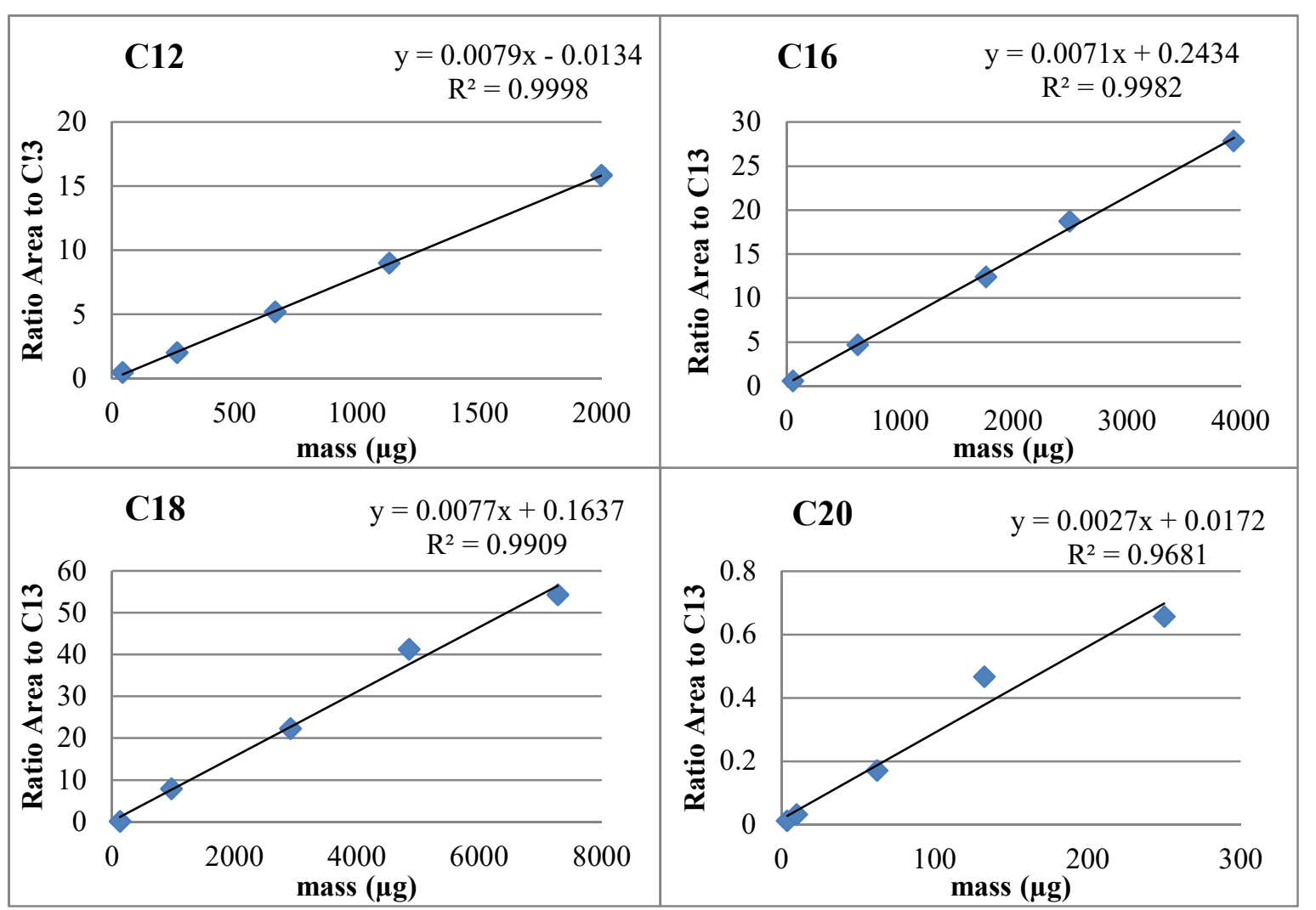

Figure 35. Calibration curves for FAMEs relative to the internal standard with different chain lengths (a) C12, (b) C16, (c) C18, and (d) C20. Amounts used are shown in Table 7.

The response factors for all of the fatty acids identified were calculated using the peak areas and the amounts in the standard mixture using Eq. 1. The factor response for peak 31 (C11:0, undecanoic methyl ester) was not calculated because it is not identified in human milk. Factor response for peak 32 (C18:2n-6t, linoleladic methyl ester) was not calculated because it was noticed that it could co-elute with nonadecanoic methyl ester, 
as $\mathrm{C} 18: 2 \mathrm{n}-6 \mathrm{t}$ is present in very low concentrations in human milk. Linear regression fits for four different standard FAMEs relative to the internal standard showed a good linearity response as shown in Figure 35.

The concentration ranges for the four standards tested were different. They were chosen to fall within the ranges reported to be found in mature milk [104]. The regression lines show a strong correlation in all cases and we can confirm the linearity of the FAMES relative to the internal standard in the ranges chosen for each standard FAME.

Table 19. Repeatability measurements from five replicates (mean $\pm \% \mathrm{RSD}$ ).

\begin{tabular}{|l|c|c|l|c|c|}
\hline & $\begin{array}{c}\text { Mean } \\
(\boldsymbol{\mu g} / \mathbf{1 0 0} \boldsymbol{\mu L})\end{array}$ & \% RSD & & $\begin{array}{c}\text { Mean } \\
(\boldsymbol{\mu g} / \mathbf{1 0 0} \boldsymbol{\mu L})\end{array}$ & \% RSD \\
\hline C10:0 & 66.00 & 5.78 & $\mathbf{C 1 8 : 3 n - 3}$ & 49.17 & 2.77 \\
\hline C12:0 & 405.51 & 3.37 & $\mathbf{C 2 0 : 1 n - 9}$ & 7.65 & 7.98 \\
\hline C14:0 & 466.49 & 1.62 & $\mathbf{C 2 1 : 0}$ & 2.48 & 8.97 \\
\hline C14:1n-5 & 4.47 & 6.44 & $\mathbf{C 2 0 : 2 n - 6}$ & 15.84 & 8.49 \\
\hline C15:0 & 6.76 & 9.06 & $\mathbf{C 2 2 : 0}$ & 1.59 & 6.01 \\
\hline C15:1n-5 & 2.66 & 9.56 & $\mathbf{C 2 0 : 3 n - 3}$ & 11.21 & 6.55 \\
\hline C16:0 & 753.79 & 1.62 & $\mathbf{C 2 0 : 3 n - 6}$ & 1.83 & 5.42 \\
\hline C16:1n-7 & 69.60 & 1.16 & $\mathbf{C 2 0 : 4 n - 6}$ & 18.14 & 8.27 \\
\hline C17:0 & 7.66 & 2.48 & $\mathbf{C 2 3 : 0}$ & 2.28 & 6.63 \\
\hline C17:1n-7 & 5.64 & 6.24 & $\mathbf{C 2 2 : 2 n - 6}$ & 2.61 & 8.47 \\
\hline C18:0 & 148.59 & 4.25 & $\mathbf{C 2 0 : 5 n - 3}$ & 3.15 & 9.95 \\
\hline C18:1t9 & 8.73 & 5.34 & $\mathbf{C 2 4 : 0}$ & 2.57 & 6.80 \\
\hline C18:1n9 & 704.17 & 3.31 & $\mathbf{C 2 4 : 1 n - 9}$ & 1.80 & 3.71 \\
\hline C18:2n6 & 591.46 & 3.59 & $\mathbf{C 2 2 : 6 n - 3}$ & 13.00 & 9.27 \\
\hline C20:0 & 6.00 & 4.31 & & & \\
\hline
\end{tabular}

The precision of our method was calculated for repeatability using one milk sample, as the matrix and the extraction was made in five replicates during the same day. 
The results are shown in Table 20. The precision was acceptable for all major fatty acids. Fatty acids that are present in the matrix sample with a concentration higher than $10 \%$ showed a low RSD between $1.6-3.6 \%$. On the other hand, the RDS was higher for those with lower concentrations $(<20 \mu \mathrm{g} / 100 \mu \mathrm{L})$. The RSD was found to be $4.3-9.3 \%$ for those fatty acids present in less than $0.4 \%$ concentration.

A calibration curve for the internal standard was plotted (Figure 36). STEYX was calculated to be 27.54 and using the slope, LOD and LOQ were calculated to be 0.27 $\mu \mathrm{g} / \mu \mathrm{L}$ and $2.73 \mu \mathrm{g} / \mu \mathrm{L}$ respectively.

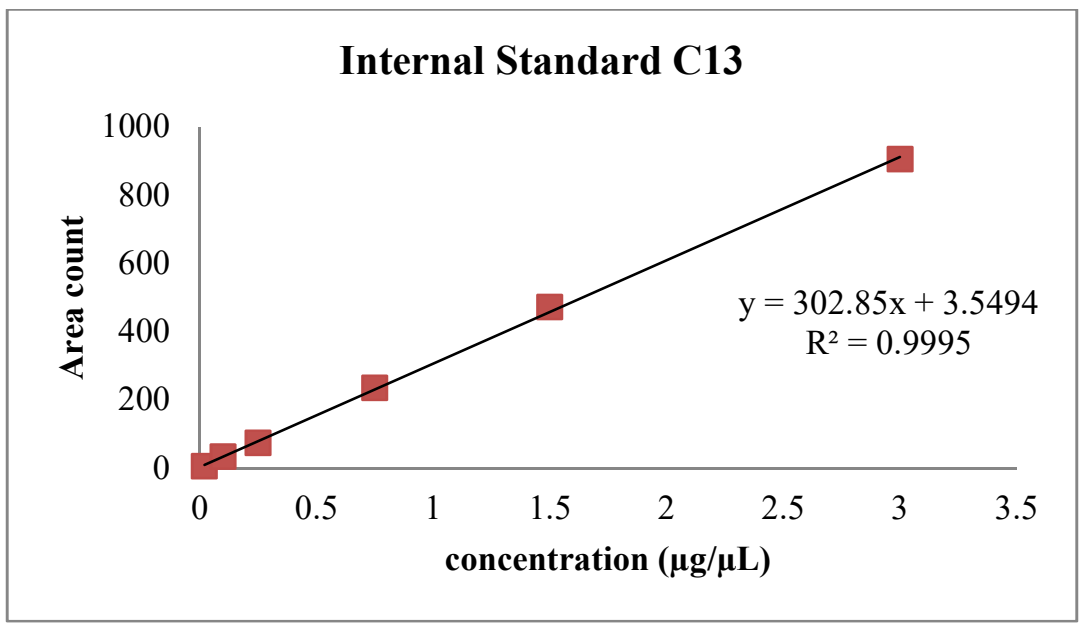

Figure 36. Calibration curve for to the internal standard $\mathrm{C} 13$.

Table 20 . Total $\%$ of fat in all milk samples during $2 \mathrm{nd}$, 3rd and 4 th week after delivery.

\begin{tabular}{|c|c|c|c|c|}
\hline & N & Range & Mean (\%) & SEM \\
\hline W2 & 30 & $1.25-5.58$ & 3.45 & 0.18 \\
\hline W3 & 28 & $0.79-5.28$ & 3.33 & 0.21 \\
\hline W4 & 74 & $1.20-8.22$ & 3.77 & 0.16 \\
\hline
\end{tabular}


Table 21. Fatty acid composition of transitional and mature milk $(\% \mathrm{w} / \mathrm{w})$ in all subjects.

\begin{tabular}{|c|c|c|c|c|c|c|c|c|c|c|c|c|}
\hline & \multicolumn{4}{|c|}{$\begin{array}{c}\text { Transitional milk } 2^{\text {nd }} \\
\text { Week } N=30\end{array}$} & \multicolumn{4}{|c|}{$\begin{array}{l}\text { Mature milk } 3^{\text {rd }} \\
\text { Week } N=28\end{array}$} & \multicolumn{4}{|c|}{$\begin{array}{l}\text { Mature milk } 4^{\text {th }} \\
\text { Week } N=74\end{array}$} \\
\hline & Min & Max & Mean & SEM & Min & Max & Mean & SEM & Min & $\operatorname{Max}$ & Mean & SEM \\
\hline C10:0 & 1.05 & 6.07 & 2.11 & 0.18 & 0.94 & 3.65 & 1.97 & 0.11 & 0.87 & 4.56 & 2.05 & 0.08 \\
\hline C12:0 & 2.85 & 18.85 & 7.59 & 0.65 & 2.74 & 12.38 & 6.79 & 0.41 & 2.97 & 15.39 & 7.42 & 0.28 \\
\hline C14:0 & 2.09 & 14.83 & 6.31 & 0.55 & 2.31 & 12.80 & 5.71 & 0.41 & 2.24 & 11.75 & 5.97 & 0.23 \\
\hline C15:0 & 0.11 & 0.46 & 0.23 & 0.02 & 0.08 & 0.35 & 0.21 & 0.01 & 0.10 & 0.48 & 0.20 & 0.01 \\
\hline C16:0 & 17.04 & 24.51 & 20.13 & 0.42 & 13.44 & 28.34 & 20.27 & 0.66 & 14.91 & 27.52 & 19.44 & 0.27 \\
\hline C17:0 & 0.17 & 0.48 & 0.30 & 0.01 & 0.19 & 0.55 & 0.30 & 0.02 & 0.20 & 0.57 & 0.30 & 0.01 \\
\hline C18:0 & 3.59 & 6.61 & 5.30 & 0.11 & 4.00 & 6.78 & 5.40 & 0.13 & 3.71 & 14.52 & 5.54 & 0.16 \\
\hline C20:0 & 0.24 & 0.50 & 0.36 & 0.01 & 0.11 & 0.58 & 0.39 & 0.02 & 0.16 & 0.63 & 0.38 & 0.01 \\
\hline C21:0 & 0.12 & 0.68 & 0.26 & 0.02 & 0.08 & 0.72 & 0.25 & 0.02 & 0.11 & 0.44 & 0.21 & 0.01 \\
\hline C22:0 & 0.10 & 1.06 & 0.20 & 0.03 & 0.11 & 0.49 & 0.20 & 0.01 & 0.09 & 0.80 & 0.19 & 0.01 \\
\hline C23:0 & 0.13 & 0.50 & 0.26 & 0.01 & 0.14 & 0.46 & 0.29 & 0.01 & 0.12 & 0.52 & 0.26 & 0.01 \\
\hline C24:0 & 0.29 & 0.74 & 0.48 & 0.02 & 0.32 & 0.86 & 0.53 & 0.02 & 0.20 & 0.94 & 0.41 & 0.01 \\
\hline total SFA & \multicolumn{4}{|c|}{43.52} & \multicolumn{4}{|c|}{42.31} & \multicolumn{4}{|c|}{42.37} \\
\hline C14:1n-5 & 0.08 & 0.37 & 0.17 & 0.01 & 0.06 & 0.33 & 0.17 & 0.01 & 0.06 & 0.62 & 0.18 & 0.01 \\
\hline C15:1n-5 & 0.08 & 0.62 & 0.21 & 0.02 & 0.12 & 0.60 & 0.29 & 0.03 & 0.05 & 0.64 & 0.25 & 0.01 \\
\hline C16:1n-7 & 1.05 & 4.95 & 2.66 & 0.16 & 1.56 & 4.57 & 2.83 & 0.17 & 1.11 & 4.75 & 2.71 & 0.09 \\
\hline C17:1n-7 & 0.10 & 0.27 & 0.17 & 0.01 & 0.11 & 0.32 & 0.19 & 0.01 & 0.09 & 0.33 & 0.18 & 0.01 \\
\hline C18:1t9 & 0.16 & 2.08 & 0.53 & 0.07 & 0.19 & 1.64 & 0.52 & 0.06 & 0.18 & 1.39 & 0.50 & 0.03 \\
\hline C18:1n-9 & 17.90 & 29.87 & 25.97 & 0.61 & 20.05 & 31.33 & 26.45 & 0.57 & 19.43 & 33.01 & 25.85 & 0.32 \\
\hline C20:1n-9 & 0.29 & 0.70 & 0.41 & 0.02 & 0.31 & 0.68 & 0.46 & 0.02 & 0.08 & 0.57 & 0.42 & 0.01 \\
\hline C24:1n-9 & 0.13 & 0.47 & 0.26 & 0.02 & 0.16 & 0.54 & 0.27 & 0.02 & 0.10 & 0.64 & 0.20 & 0.01 \\
\hline $\begin{array}{l}\text { total } \\
\text { MUFA }\end{array}$ & \multicolumn{4}{|c|}{30.38} & \multicolumn{4}{|c|}{31.19} & \multicolumn{4}{|c|}{30.29} \\
\hline C18:2n-6 & 11.48 & 29.92 & 20.46 & 0.76 & 12.80 & 32.79 & 20.95 & 0.90 & 2.91 & 28.59 & 21.87 & 0.51 \\
\hline$C 20: 2 n-6$ & 0.59 & 1.22 & 0.82 & 0.03 & 0.59 & 1.25 & 0.85 & 0.03 & 0.52 & 1.14 & 0.81 & 0.01 \\
\hline$C 20: 3 n-6$ & 0.05 & 0.98 & 0.14 & 0.03 & 0.07 & 0.96 & 0.16 & 0.03 & 0.06 & 0.90 & 0.13 & 0.01 \\
\hline C20:4n-6 & 0.11 & 1.49 & 0.98 & 0.05 & 0.73 & 1.98 & 1.03 & 0.05 & 0.58 & 1.57 & 0.85 & 0.02 \\
\hline$C 22: 2 n-6$ & 0.10 & 1.36 & 0.28 & 0.04 & 0.14 & 0.59 & 0.24 & 0.02 & 0.09 & 0.40 & 0.17 & 0.01 \\
\hline $\begin{array}{c}\text { total } \\
\text { PUFA n-6 }\end{array}$ & \multicolumn{4}{|c|}{22.69} & \multicolumn{4}{|c|}{23.23} & \multicolumn{4}{|c|}{23.83} \\
\hline C18:3n-3 & 0.85 & 2.31 & 1.59 & 0.07 & 0.15 & 2.78 & 1.52 & 0.10 & 0.66 & 2.71 & 1.81 & 0.05 \\
\hline C20:3n-3 & 0.44 & 1.34 & 0.84 & 0.04 & 0.50 & 1.51 & 0.90 & 0.04 & 0.09 & 1.43 & 0.79 & 0.02 \\
\hline C20:5n-3 & 0.05 & 0.42 & 0.15 & 0.02 & 0.06 & 0.26 & 0.12 & 0.01 & 0.04 & 0.35 & 0.12 & 0.01 \\
\hline C22:6n-3 & 0.44 & 3.37 & 0.84 & 0.10 & 0.48 & 1.51 & 0.74 & 0.05 & 0.38 & 1.99 & 0.78 & 0.04 \\
\hline $\begin{array}{c}\text { total } \\
\text { PUFA n-3 }\end{array}$ & \multicolumn{4}{|c|}{3.41} & \multicolumn{4}{|c|}{3.28} & \multicolumn{4}{|c|}{3.50} \\
\hline
\end{tabular}


Table 22. Fatty acid composition of mother's milk $(\% \mathrm{w} / \mathrm{w})$ for the same subjects.

\begin{tabular}{|c|c|c|c|c|c|c|c|c|c|c|c|c|}
\hline & \multicolumn{4}{|c|}{$\begin{array}{c}\text { Transitional milk } 2^{\text {nd }} \\
\text { Week } N=26\end{array}$} & \multicolumn{4}{|c|}{$\begin{array}{l}\text { Mature milk } 3^{\text {rd }} \\
\text { Week } N=26\end{array}$} & \multicolumn{4}{|c|}{$\begin{array}{l}\text { Mature milk } 4^{\text {th }} \\
\text { Week } N=26\end{array}$} \\
\hline & Min & $\operatorname{Max}$ & Mean & SEM & Min & Max & Mean & SEM & Min & Max & Mean & SEM \\
\hline C10:0 & 1.13 & 6.07 & 2.18 & 0.20 & 0.94 & 3.65 & 1.95 & 0.12 & 1.35 & 4.56 & 2.27 & 0.16 \\
\hline C12:0 & 4.14 & 18.85 & 7.85 & 0.71 & 2.74 & 12.38 & 6.87 & 0.43 & 3.22 & 15.39 & 7.78 & 0.58 \\
\hline C14:0 & 2.87 & 14.83 & 6.45 & 0.59 & 2.31 & 12.80 & 5.86 & 0.43 & 2.24 & 11.42 & 6.09 & 0.47 \\
\hline C15:0 & 0.11 & 0.43 & 0.22 & 0.02 & 0.11 & 0.35 & 0.21 & 0.01 & 0.12 & 0.38 & 0.20 & 0.01 \\
\hline C16:0 & 17.04 & 24.07 & 20.02 & 0.43 & 16.27 & 28.34 & 20.57 & 0.65 & 14.97 & 23.50 & 19.66 & 0.46 \\
\hline C17:0 & 0.17 & 0.48 & 0.29 & 0.02 & 0.19 & 0.55 & 0.31 & 0.02 & 0.20 & 0.40 & 0.29 & 0.01 \\
\hline C18:0 & 3.59 & 5.92 & 5.21 & 0.11 & 4.00 & 6.78 & 5.42 & 0.14 & 4.20 & 8.23 & 5.32 & 0.18 \\
\hline C20:0 & 0.24 & 0.50 & 0.36 & 0.01 & 0.11 & 0.58 & 0.39 & 0.02 & 0.27 & 0.63 & 0.40 & 0.02 \\
\hline C21:0 & 0.12 & 0.68 & 0.26 & 0.03 & 0.08 & 0.72 & 0.25 & 0.02 & 0.12 & 0.34 & 0.22 & 0.01 \\
\hline C22:0 & 0.10 & 1.06 & 0.20 & 0.04 & 0.11 & 0.49 & 0.20 & 0.02 & 0.09 & 0.80 & 0.19 & 0.03 \\
\hline C23:0 & 0.13 & 0.37 & 0.25 & 0.01 & 0.14 & 0.46 & 0.29 & 0.02 & 0.14 & 0.52 & 0.29 & 0.02 \\
\hline C24:0 & 0.29 & 0.74 & 0.46 & 0.02 & 0.32 & 0.86 & $0.53^{\mathrm{b}}$ & 0.02 & 0.31 & 0.94 & 0.44 & 0.03 \\
\hline total SFA & \multicolumn{4}{|c|}{43.75} & \multicolumn{4}{|c|}{42.86} & \multicolumn{4}{|c|}{43.14} \\
\hline C14:1n-5 & 0.08 & 0.37 & 0.17 & 0.01 & 0.06 & 0.33 & 0.17 & 0.01 & 0.09 & 0.32 & 0.17 & 0.01 \\
\hline C15:1n-5 & 0.08 & 0.62 & $0.21^{\mathrm{a}}$ & 0.02 & 0.12 & 0.60 & 0.30 & 0.03 & 0.09 & 0.48 & 0.24 & 0.02 \\
\hline C16:1n-7 & 1.05 & 4.95 & 2.67 & 0.18 & 1.56 & 4.57 & 2.87 & 0.18 & 1.30 & 4.38 & 2.59 & 0.17 \\
\hline C17:1n-7 & 0.10 & 0.27 & 0.17 & 0.01 & 0.13 & 0.32 & 0.19 & 0.01 & 0.09 & 0.26 & 0.17 & 0.01 \\
\hline C18:1t9 & 0.16 & 2.08 & 0.53 & 0.07 & 0.22 & 1.64 & 0.54 & 0.07 & 0.18 & 1.19 & 0.42 & 0.05 \\
\hline C18:1n-9 & 17.90 & 29.87 & 25.93 & 0.64 & 20.05 & 31.33 & 26.38 & 0.59 & 19.43 & 29.92 & 25.24 & 0.55 \\
\hline C20:1n-9 & 0.29 & 0.70 & 0.41 & 0.02 & 0.31 & 0.68 & $0.46^{\mathrm{b}}$ & 0.02 & 0.08 & 0.53 & 0.40 & 0.02 \\
\hline C24:1n-9 & 0.13 & 0.47 & 0.25 & 0.02 & 0.16 & 0.54 & 0.28 & 0.02 & 0.10 & 0.64 & 0.22 & 0.02 \\
\hline $\begin{array}{c}\text { total } \\
\text { MUFA }\end{array}$ & \multicolumn{4}{|c|}{30.34} & \multicolumn{4}{|c|}{31.18} & \multicolumn{4}{|c|}{29.45} \\
\hline C18:2n-6 & 11.48 & 26.91 & 20.34 & 0.78 & 12.80 & 27.50 & 20.41 & 0.84 & 14.44 & 28.59 & 21.96 & 0.79 \\
\hline C20:2n-6 & 0.59 & 1.22 & 0.81 & 0.03 & 0.59 & 1.25 & 0.83 & 0.03 & 0.52 & 1.12 & 0.76 & 0.03 \\
\hline$C 20: 3 n-6$ & 0.05 & 0.98 & 0.15 & 0.03 & 0.07 & 0.96 & 0.16 & 0.03 & 0.06 & 0.20 & 0.11 & 0.01 \\
\hline$C 20: 4 n-6$ & 0.11 & 1.49 & 0.99 & 0.05 & 0.73 & 1.98 & $1.04^{\mathrm{b}}$ & 0.05 & 0.62 & 1.57 & 0.89 & 0.04 \\
\hline$C 22: 2 n-6$ & 0.10 & 1.36 & 0.28 & 0.05 & 0.14 & 0.59 & 0.24 & 0.02 & 0.10 & 0.40 & 0.20 & 0.01 \\
\hline $\begin{array}{c}\text { total } \\
\text { PUFA n-6 }\end{array}$ & \multicolumn{4}{|c|}{22.57} & \multicolumn{4}{|c|}{22.69} & \multicolumn{4}{|c|}{23.91} \\
\hline C18:3n-3 & 0.85 & 2.31 & 1.58 & 0.08 & 0.15 & 2.40 & $1.49^{\mathrm{b}}$ & 0.10 & 0.79 & 2.65 & 1.80 & 0.09 \\
\hline$C 20: 3 n-3$ & 0.44 & 1.34 & 0.86 & 0.05 & 0.50 & 1.51 & $0.91^{b}$ & 0.05 & 0.09 & 1.43 & 0.80 & 0.05 \\
\hline C20:5n-3 & 0.05 & 0.42 & 0.14 & 0.02 & 0.07 & 0.26 & 0.13 & 0.01 & 0.04 & 0.32 & 0.13 & 0.01 \\
\hline C22:6n-3 & 0.44 & 1.56 & 0.76 & 0.05 & 0.48 & 1.51 & 0.75 & 0.05 & 0.39 & 1.99 & 0.75 & 0.07 \\
\hline $\begin{array}{c}\text { total } \\
\text { PUFA n-3 }\end{array}$ & \multicolumn{4}{|c|}{3.34} & \multicolumn{4}{|c|}{3.27} & \multicolumn{4}{|c|}{3.49} \\
\hline
\end{tabular}

${ }^{a}$ Significantly different $(p<0.05)$ between transitional and mature milk from $3^{\text {rd }}$. week 
${ }^{\text {b }}$ Significantly different $(\mathrm{p}<0.05)$ between mature milk from $3^{\text {rd }}$. and $4^{\text {th }}$. week

The results of total \% fat analyzed in all the 74 subjects are shown in Table 21. The average results for the extraction of FAMEs from milk samples from weeks 2, 3 and 4 after delivery are shown in Table 22. Data were collected in triplicates for a group of 26 mothers during three consecutive weeks. The results for the FAME extractions and total \% fat of the previously mentioned group are shown in Tables 23 and 24, respectively.

Table 23 . Total $\%$ of fat in milk samples during week 2,3 and 4 th after delivery for the same subjects.

\begin{tabular}{|c|c|c|c|c|}
\hline & $\mathbf{N}$ & Range & Mean (\%) & SEM \\
\hline W2 & 26 & $1.25-5.58$ & 3.40 & 0.20 \\
\hline W3 & 26 & $0.79-5.28$ & 3.26 & 0.22 \\
\hline W4 & 26 & $1.20-6.43$ & 3.26 & 0.28 \\
\hline
\end{tabular}

No significant difference was found for the total $\%$ of fat among the three weeks studied. Only 5 FAs (C24:0, C20:1n-9, C20:4n-6, C18:3n-3, C20:3n-3) were significantly different between week 3 and week 4 of lactation. ALA (C18:3n-3) is the only member of this group that is an essential fatty acid. The rest are long chain fatty acids. In addition, there was one MUFA, C15:1n-5, which showed a significant difference between transitional milk (week 2) and mature milk form the $3^{\text {rd }}$ week. Average results from the frequency intake of food from animal sources are shown in Table 25. 
Table 24. Food frequency intake.

\begin{tabular}{|c|cccc|}
\hline \multicolumn{5}{|c|}{ Food frequency during one month $\mathbf{N}=\mathbf{7 4}$} \\
\hline & Min & Max & Average (times per month) & Std. dev. \\
\hline Chicken, poultry & 4 & 63 & 29.35 & 13.20 \\
Red meat & 0 & 63 & 5.51 & 8.81 \\
Chicken's liver & 0 & 60 & 8.39 & 10.93 \\
Cow's liver & 0 & 8 & 0.72 & 1.68 \\
Fish & 0 & 34 & 5.77 & 6.68 \\
Sea food & 0 & 9 & 0.99 & 2.02 \\
Dairy products (milk, & 0 & 120 & 39.50 & 28.53 \\
cheese, and yogurt) & 0 & 42 & 15.28 & 10.19 \\
Eggs & & &
\end{tabular}

\subsection{Discussion and Conclusions}

This study evaluated the fatty acid profiles of 74 healthy Peruvian women with an omnivorous diet. Comparable replicated measurements for 26 subjects from the available population sample were evaluated longitudinally to compare FA variation over three weeks during the first month of lactation. The results from the latter group are presented in Table 23. A two-way ANOVA test was run and the results showed a significant difference for six fatty acids (see Table 26).

Table 25. FAs with a significant difference between weeks 2 and 4 of lactation.

\begin{tabular}{|l|l|l|l|}
\hline Fatty acid & $\mathrm{p}$ value & Groups & Change \\
\hline C24:0 & 0.016 & $3^{\text {rd }}$ week $\& 4^{\text {th }}$ week & Decreased \\
\hline C15:1n-5 & 0.034 & $2^{\text {nd }}$ week $\& 3^{\text {rd }}$ week & Increased \\
\hline C20:1n-9 & 0.027 & $3^{\text {rd }}$ week $\& 4^{\text {th }}$ week & Decreased \\
\hline C20:4n-6 AA & 0.011 & $3^{\text {rd }}$ week \& $4^{\text {th }}$ week & Decreased \\
\hline C18:3n-3 ALA & 0.008 & $3^{\text {rd }}$ week \& $4^{\text {th }}$ week & Increased \\
\hline C20:3n-3 & 0.019 & $3^{\text {rd }}$ week \& $4^{\text {th }}$ week & Decreased \\
\hline
\end{tabular}


Only one MUFA (C15:1n-5) showed a difference between transitional $\left(2^{\text {nd }}\right.$ week) and mature milk ( $3^{\text {rd }}$ week). The other FA showed significant differences between mature milk during the $3^{\text {rd }}$ and $4^{\text {th }}$ weeks. All of these were long chain fatty acids and one of them is the essential fatty acid ALA (C18:3n-3).

In the literature we find that total lipid levels are usually reported to increase with time during lactation [75]. We did not see a consistent significant increase in the total fat content in milk during the 3 week period of this study. Studies $[119,121,134]$ have shown that the largest variation of FA levels in human milk occurs during the transitional period from colostrum to mature milk. Another longitudinal study [135] have reported an increase from colostrum to transitional and then a slightly decrease from transitional to mature milk, all only within the first month of lactation. Our results agree and show that there is a decrease from transitional milk to mature milk. Most longitudinal studies have not reported the variations during the first month week by week to form a basis for a general conclusion. Our study is unique in that we were able to study the variations week by week during the first month.

Significant variation between transitional $\left(2^{\text {nd }}\right.$ week) and mature milk $\left(4^{\text {th }}\right.$ week or later) has been reported for only a few FA and shows an influence from the mothers' diet. In general, results showing significant longitudinal differences in total milk fat and individual components are not universally reported. We suspect that for our study the few significant differences are most probably due to the mothers' diet. In particular, we note that ALA is of dietary origin and its level in milk varies among regional populations in a manner that reflects dietary intake from our review above. 
Table 26. Total amounts $(\% \mathrm{w} / \mathrm{w})$ variation during the first month by type of FA.

\begin{tabular}{|c|cc|c|}
\hline & $\begin{array}{c}\text { Initial week } \\
\text { (transitional milk) }\end{array}$ & $\begin{array}{c}\text { Last week } \\
\text { (mature milk) }\end{array}$ & $\% \Delta$ \\
\hline total SFA & 43.75 & 43.14 & -1.39 \\
total MUFA & 30.34 & 29.45 & -2.93 \\
total PUFA n-6 & 22.57 & 23.91 & 5.96 \\
total PUFA n-3 & 3.34 & 3.49 & 4.59 \\
\hline
\end{tabular}
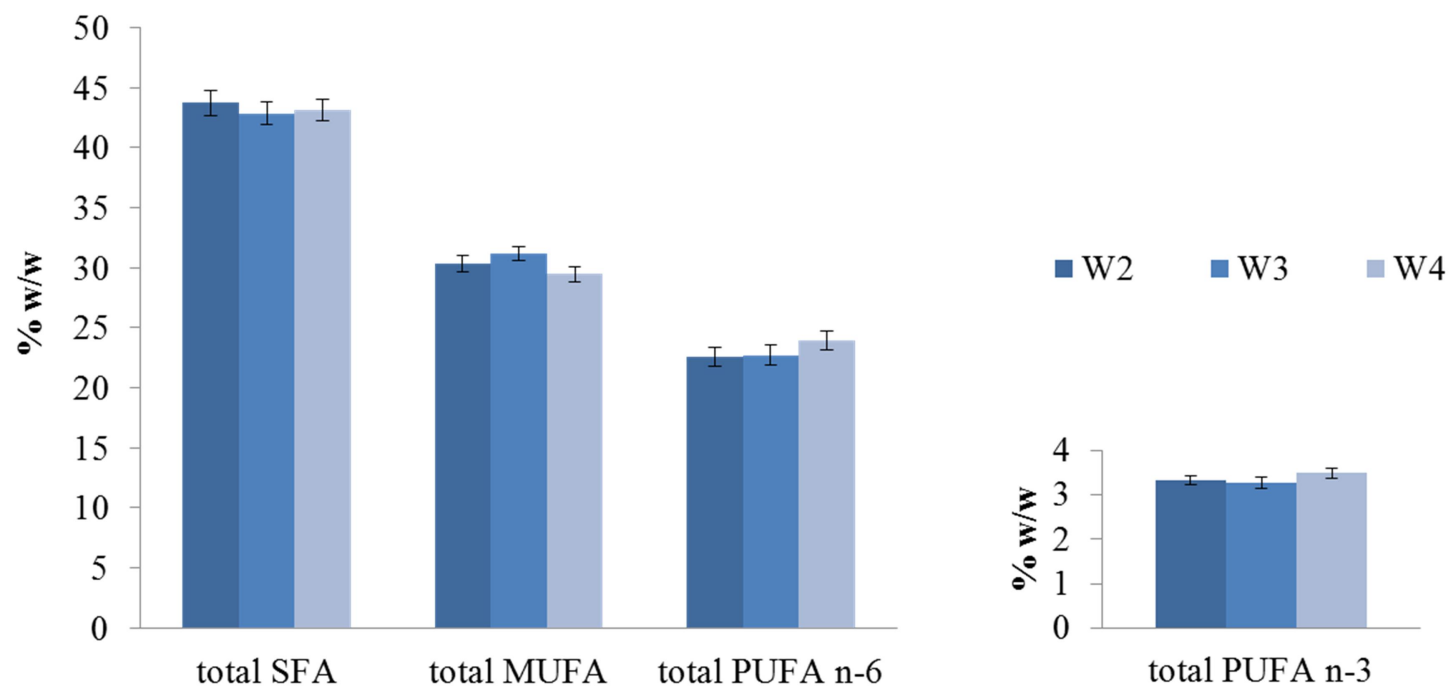

Figure 37 . Total amounts $(\% \mathrm{w} / \mathrm{w})$ variation during the first month by type of FA.

When comparing the total \% levels for each group by fatty acid type (see Figure 37 ), we found that there is a variation between the $2^{\text {nd }}$ week (transitional) and 4 th week (mature milk). Although the values of SFA and MUFA decreased, it was not by significant amounts. The amounts of n-6 and n-3 PUFAs increased $6.0 \%$ and $4.6 \%$ respectively, see Table 27 above.

As seen, with the exception of six FA mentioned above, the amounts of the FAs evaluated were not observed to be significantly different among the three weeks of lactation studied $\left(2^{\text {nd }}-4^{\text {th }}\right.$ week) Recognizing this, we have analyzed results from the 
fourth week of lactation, see Table 14, which represents a much larger sample population (all 74 subjects). These data enable us to provide a quantitative description of the FA profiles in the milk from this Peruvian population.
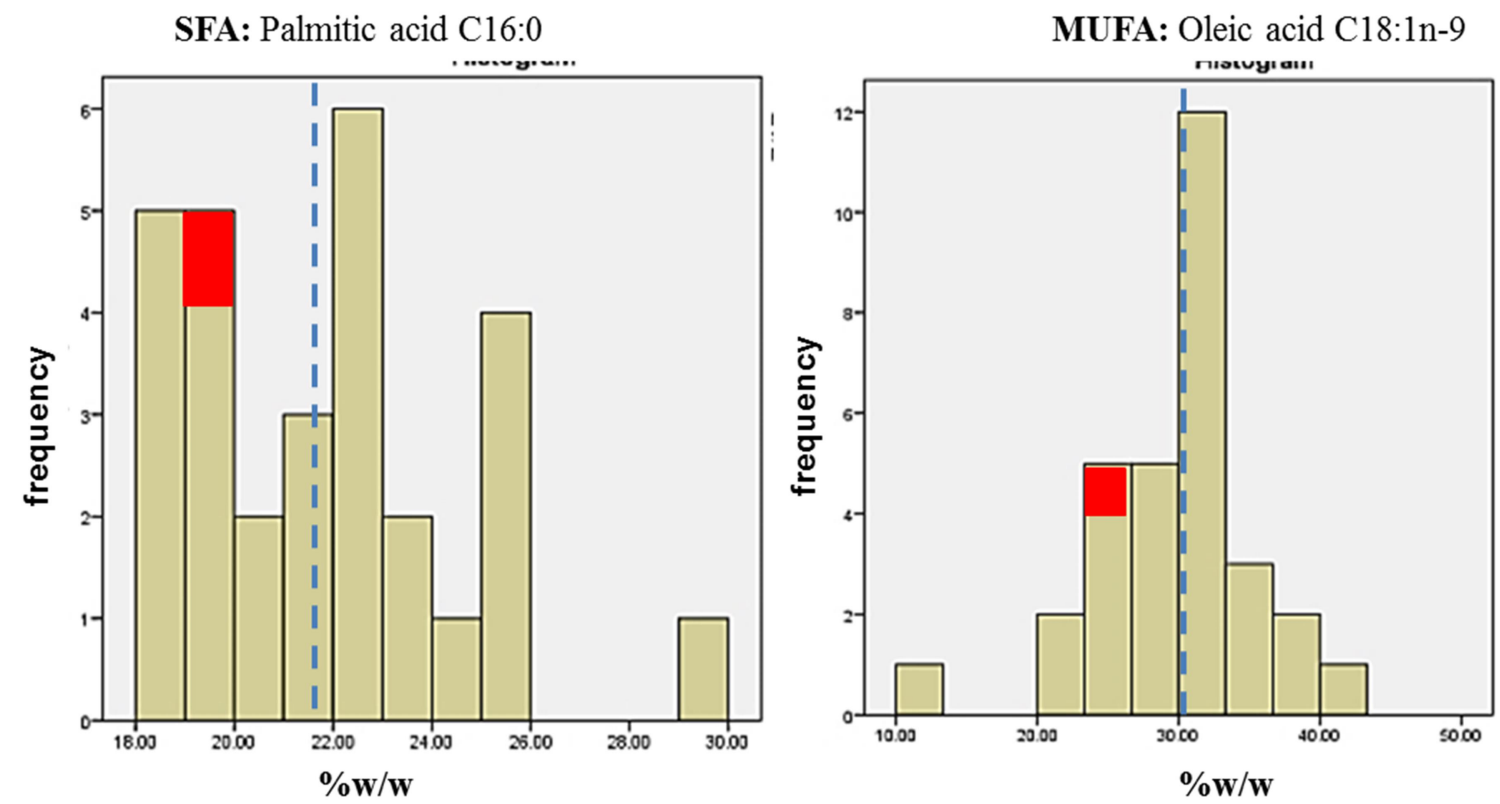

Figure 38. Histogram, all countries including Peru, for A) Palmitic acid, Mean $=21.77 \%$, $\mathrm{N}=29$. B) Oleic acid, Mean $=30.3 \%, \mathrm{~N}=31$. Blue dashed line represents the mean of all countries. Red square represents Peru.

SFA constitutes $42.37 \%$ of the total fatty acids found in milk of the Peruvian mothers in our study, results which are similar to levels found in many countries in Europe, mean $=42.15 \%$ and South America, mean $=42.18 \%($ see Appendix 1 and 2). The most abundant SFA in milk was Palmitic acid with $19.44 \%$ and accounts for almost half of the total SFAs. The amount of palmitic acid in milk of Peruvian mother's is lower than the mean of that for all countries compared in our review of the literature (see Figure 38) but falls within the range found for all countries in the Americas (South and North). The level of palmitic acid although lower, is similar to that found in Chinese and 
Japanese mothers (see Appendix 4). In our study the amount of stearic acid, 5.54\%, was found to be near the average when compared with many countries studied worldwide (mean level is $5.95 \%$ ). The levels of the fatty acids that are synthesized de novo in the mammary ducts (capric $=2.05 \%$, lauric $=7.42 \%$, and myristic acid $=5.97 \%$ ) are all found within the standard ranges seen in populations from other South American countries (Chile and Brazil). They comprise a little more than one third of the total SFA.

MUFA account for $30.29 \%$ of total fatty acids in milk of Peruvian mothers, which is lower than the range for values found in other South American countries (31.20$34.41 \%)$. The major component is oleic acid (25.85\%) and the level was lower than in all countries in Europe, North America, Australia, China and Japan, see Figure 38. The content of oleic acid in the milk of Peruvian mothers falls in the lowest quartile of the range for all the countries reviewed. The oleic acid content is close to the levels found in the milk of Brazilian, Chilean, and Brazilian mothers. This finding may reflect of a lower intake of animal fats and olive oil than in Mediterranean countries such as Italy and Spain. The finding is also consistent with a lower intake of soybean oil and other soy derived products that are common in diets of mothers in Asian countries.

Table 27. Elaidic acid in human milk in different countries $(\% \mathrm{w} / \mathrm{w})$.

\begin{tabular}{|c|c|c|c|c|c|c|c|c|}
\hline $\begin{array}{c}\text { Country } \\
\text { Author } \\
\text { [reference] }\end{array}$ & $\begin{array}{c}\text { Italy } \\
\text { Haddad } \\
{[95]}\end{array}$ & $\begin{array}{c}\text { Germany } \\
\text { Precht } \\
{[101]}\end{array}$ & $\begin{array}{c}\text { Bolivia } \\
\text { Martin } \\
\text { [114] }\end{array}$ & $\begin{array}{c}\text { Turkey } \\
\text { Samur } \\
{[136]}\end{array}$ & $\begin{array}{c}\text { US } \\
\text { Martin } \\
{[114]}\end{array}$ & $\begin{array}{c}\text { Neatherlands } \\
\text { Mueller } \\
\text { [137] }\end{array}$ & $\begin{array}{c}\text { Brazil } \\
\text { Barenhauser } \\
{[134]}\end{array}$ & $\begin{array}{c}\text { Peru } \\
\text { OUR } \\
\text { STUDY }\end{array}$ \\
\hline $\mathbf{N}$ & 8 & 40 & 35 & 50 & 35 & 185 & 10 & 74 \\
\hline $\begin{array}{l}\text { lactation } \\
\text { stage }\end{array}$ & $\begin{array}{l}>15 \\
\text { day }\end{array}$ & $<8$ days & 3-6 mo. & 3-4 mo & $>6 \mathrm{mo}$ & $1 \mathrm{mo}$. & $>16$ day & 3 week \\
\hline C18:1t9 & 1.21 & 0.37 & 0.21 & 1.96 & 1.23 & 0.61 & 1.67 & 0.50 \\
\hline
\end{tabular}

The only TFA measured in our study was elaidic acid $(\mathrm{C} 18: 1, \mathrm{t} 9)$. There are a small number of publications that include elaidic acid in their results. A comparison of 
our results with those from other populations is presented in Table 27. Many of the studies have compared the results of TFA in human milk with diet, and have concluded that eating habits determine the levels of TFA. TFAs in different populations are markers of the influence of processed, high fat content foods. Mueller et al.[137] indicated that a diet rich in dairy products and/or meat and dairy of organic origin was associated with a lower ratio of elaidic acid to $\mathrm{C} 18: 1 \mathrm{t} 11$ in milk. It was suggested that this isomer ratio be taken into account when studying the health-related impact of TFA levels in foods. The amount of elaidic acid found in the milk of Peruvian mothers was found to be less than half the level of US mothers and supports the conclusion that the intake of TFA in the diet of the Peruvian donors has a lower intake of TFAs from processed food than in the US. During recent decades, the intake of trans-fat has continuously decreased in Western diets. In the US, between 2003 and 2006, trans fat intake dropped to 1.3 grams per person per day from 4.6 grams per person per day due to new regulations restricting use of trans-fat by the food industry [138]. Thus, when using TFAs as markers in different populations, we could assume that the high level of elaidic acid is caused by the influence of processed and high fat content food in the American diet as well as in other countries. Although the influence of TFA in the new born development is not well understood, recently attention has focused on trying to determine if the conversion of LC-PUFAs from the essential FA is reduced by the presence of higher levels of TFA. If this is found to be so, it may compromise the neural development in the new born infants.

The levels of essential fatty acids in mothers' milk are of special interest because of their importance as components of the membrane lipids critical for retina and brain development. The levels of LA and ALA found in the Peruvian mothers' milk were 
$21.87 \%$ and $1.81 \%$, respectively. Both of these are in the highest quartile of distribution among the countries studied (see Figure 39). In more than $50 \%$ of all the populations reviewed before, LA fell under $15 \% \mathrm{w} / \mathrm{w}$, and more than $65 \%$ of the population fell under $1.25 \% \mathrm{w} / \mathrm{w}$ for ALA. The food questionnaire conducted as part of our study revealed that the consumption of poultry by these mothers was almost six times more frequent than the comsumption of red meat. This dietary choice is consistent with our observation of a higher content of PUFAS in Peruvian mothers' milk. . In addition, in Peru, vegetable oils were found to be preferable to olive oil in the diet, and corn is also frequently in the $\operatorname{diet}[139]$.
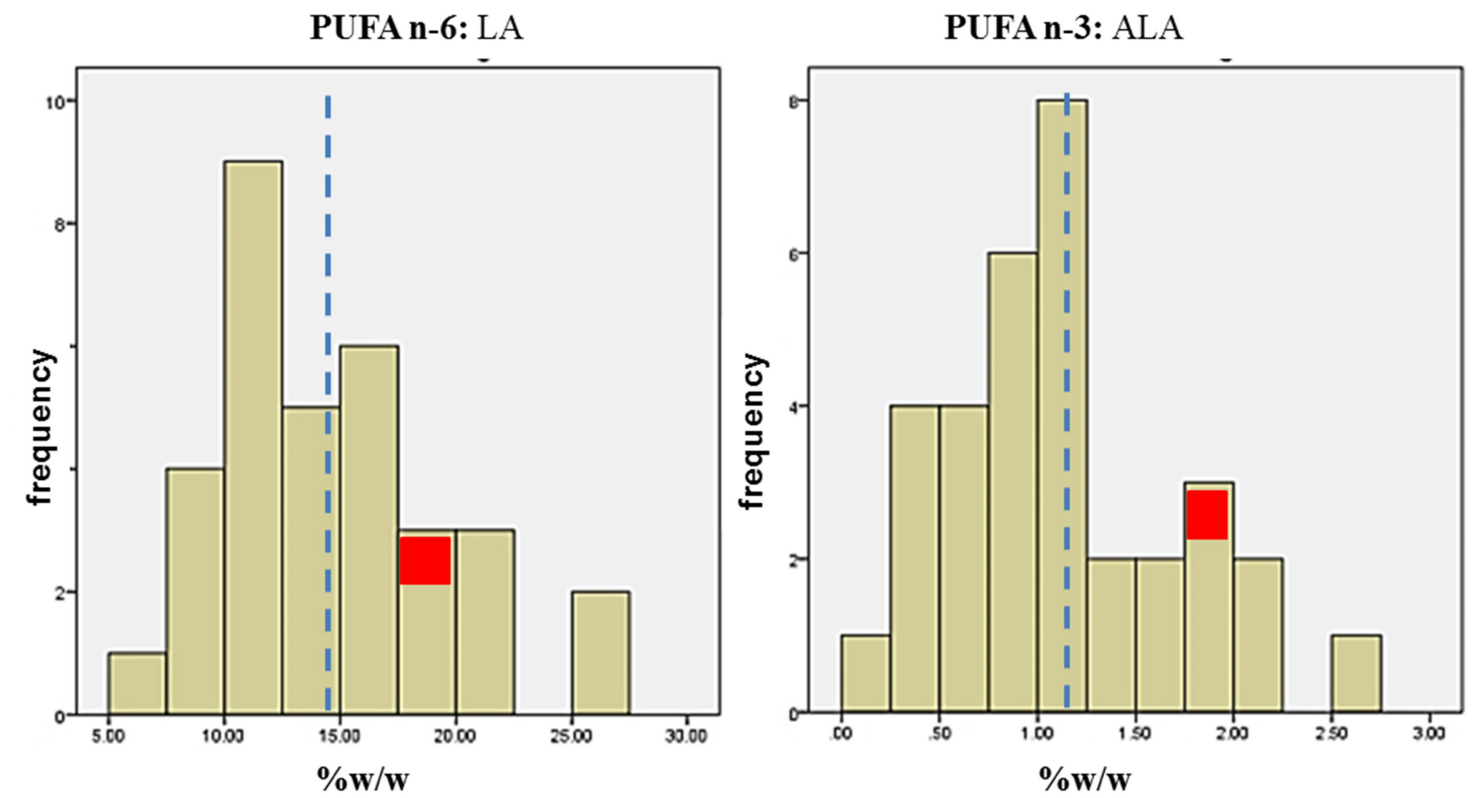

Figure 39. Histogram, all countries reviewed, for A) Linoleic acid, Mean $=14.08 \%$, Median $=13.8 \%, \mathrm{~N}=33$. B) $\alpha$-linoleic acid, Mean $=1.12 \%$, Median $=1.05 \%, \mathrm{~N}=33$. Blue dashed line represents the mean of all countries. Red square represents Peru.

Figure 40 shows the ratios of LA to ALA for the countries reviewed here. These ratios vary from 4.10 to 57.75 . The higher ratios represent larger amounts of LA in milk. 
This ratio is important since both LA and ALA compete for the same chain elongation/desaturation enzymes and high levels of n-6 LA could significantly diminish production of essential n-3 EPA and DHA needed by infants for optimal neural development. Higher ratios are indicative of an imbalanced diet resulting in lower n-3 PUFA biosynthesis. Some researchers have suggested that acceptable LA : ALA ratios fall in the range of 5:1-15:1[104], values that have been adopted in European regulations. The LA:ALA ratio found for Peruvian mothers in this study was 12.08 and although at the higher end of this range, it lies within these guidelines.

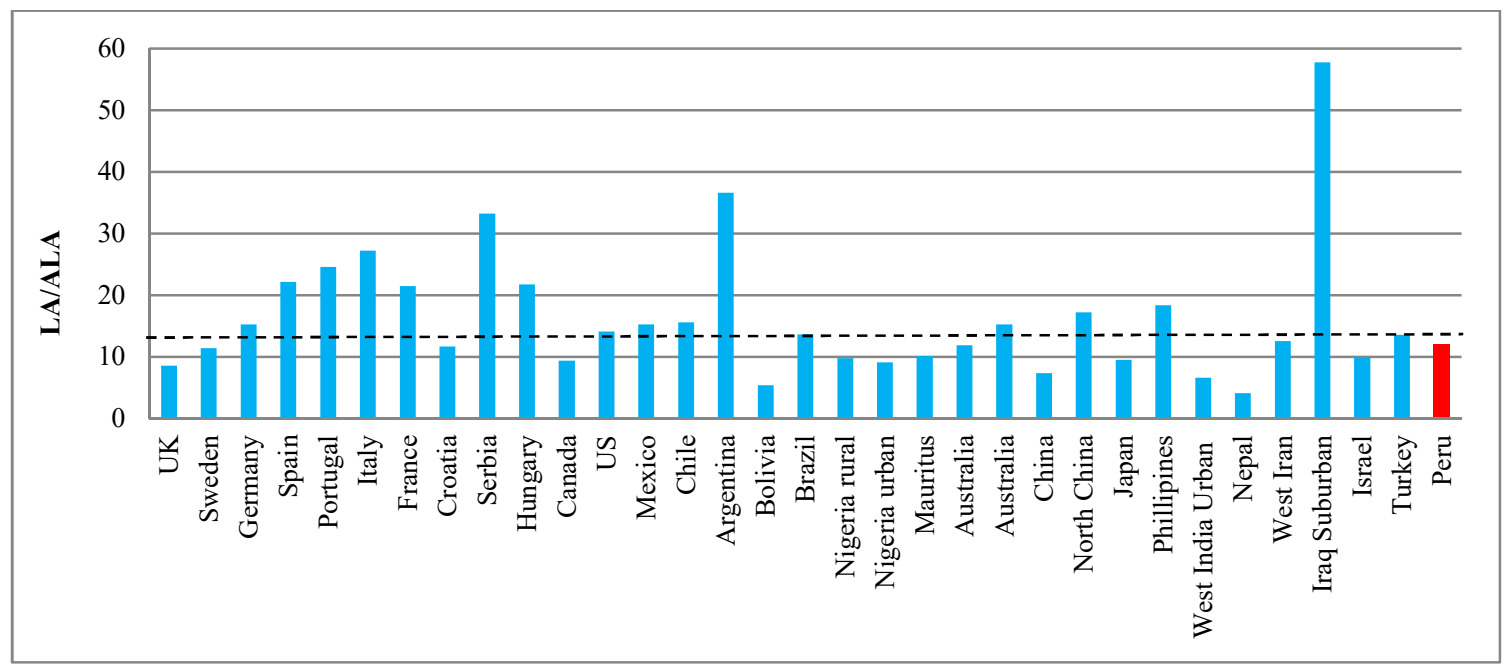

Figure 40. LA:ALA ratios for different countries. Peru is represented in red. Dashed line represents the median of all countries $=13.6$.

LC-PUFAs in the milk of Peruvian mothers were found at relatively high levels. In this study, EPA was found to average $0.12 \%$ (range $=0.03-0.39 \%$ ), DHA was $0.78 \%$ (range $=0.06-1.40 \%)$ and $\mathrm{AA}$ was $0.85 \%($ range $=0.161 .4 \%)$. Comparing these values to recent literature data (see Table 16 and Brenna et al 2007 [126]), both AA and DHA fall 
in the highest quartile compared to other populations. Despite the fact that the ratio of LA/ALA was 12:1, the ratio of the essential FAs, AA/DHA, was 1:1. The conversion of LA and ALA to AA and DHA is apparently not high, as evidenced by the ratio of AA/DHA. Instead, the result shows that both LC-PUFAs originate not only from the synthesis taking place in the mammarian glands from the uptake of LA and ALA, but also from transference from diet intake.

Table 28. DHA content $(\% \mathrm{w} / \mathrm{w})$ in human milk of US women in studies conducted from 1992-2012. Taken from Brenna et al.[126] and Martin et al.[114].

\begin{tabular}{|c|c|c|}
\hline Year & Site & DHA \% \\
\hline 1992 & Connecticut & 0.37 \\
\hline 1998 & N.S. & 0.20 \\
\hline 2000 & N.S. & 0.15 \\
\hline 2003 & Oregon & 0.20 \\
\hline 2005 & Texas & 0.20 \\
\hline 2005 & North Carolina & 0.21 \\
\hline 2006 & N.S. & 0.17 \\
\hline 2012 & Cincinnati & 0.16 \\
\hline
\end{tabular}

N.S. $=$ not specified

The amount of DHA in the milk of our Peruvian study population $(0.78 \%)$ corresponds to samples taken during 2013-2014 in Peruvian mothers. The level of DHA is anticipated to be associated with the average frequency for intake of fish and sea food of 6.8 times per month obtained from our food frequency questionnaire (see Table 24). We have compared the amount of DHA found in the Peruvian population to other studies completed in analyzing different geographical regions in the US between 1992 and 2012, see Table 28 . The median amount of DHA in the US is $0.20 \% \mathrm{w} / \mathrm{w}$. The $\%$ DHA in the 
Peruvian population is almost 4 times greater than that found most of the studies for U.S. women. One exception is a study in Connecticut women. According to the Peruvian National Institute of Statistics and Informatics, the average fish and sea food intake per capita was $10.2-11.1 \mathrm{~kg} /$ year in 2009 in the costal urban area of Peru. In comparison according to the National Oceanic and Atmospheric Organization of the U.S., the average American consumed $7.2 \mathrm{~kg}$ of fish and shellfish in same year. For reference we note that the annual Japanese consumption in 2013 was $27.3 \mathrm{~kg} / \mathrm{year}$. These data lead us to assume that in the Peruvian coastal region intake of sea food is about $30 \%$ greater than the average intake in the U.S. The high level of DHA found in milk of Peruvian women is most probably caused by the relatively high consumption of fish and sea food in their diet.

Table 29. FA concentrations $(\mathrm{mg} / \mathrm{L})$ from Peruvian mothers in mature milk (4th week of lactation). $\mathrm{N}=74$.

\begin{tabular}{|c|ccccc|}
\hline & Min. & Max. & Mean & SEM & Median \\
\hline LA & 1085.5 & 19203.5 & 8392.8 & 463.1 & 7786.5 \\
ALA & 163.7 & 1808.8 & 691.1 & 38.6 & 627.7 \\
AA & 134.6 & 641.1 & 314.6 & 13.2 & 294.2 \\
EPA & 14.3 & 126.7 & 42.5 & 2.2 & 38.6 \\
DHA & 89.4 & 903.8 & 291.1 & 18.3 & 259.4 \\
total n-6 & 1353.7 & 20801.7 & 9122.2 & & \\
total n-3 & 299.4 & 3560.1 & 1319.1 & & \\
\hline
\end{tabular}

The levels of n-6 and n-3 fatty acids are vital for the optimal development of healthy infants. The total amounts of $n-6$ and $n-3$ fatty acids in the Peruvian population studied were $23.83 \%$ and $3.50 \%$. Although it is not yet clear what the ideal intake of 
essential fatty acids from mothers' milk needed for an optimal development is, the Food and Nutrition Board of the U.S. Institute of Medicine[140] has established that the adequate daily intake (ADI) of n-6 and n-3 fatty acids for infants between 0-6 months should be $4.4 \mathrm{~g} /$ day and $0.5 \mathrm{~g} /$ day, respectively. For infants $7-12$ months the ADI has been set at $4.6 \mathrm{~g} /$ day and $0.5 \mathrm{~g} / \mathrm{day}$. The European Union regulations[141] for infant formula establishes intakes of LA at $500-1200 \mathrm{mg} / 100 \mathrm{kcal}$ and for ALA as $50-100 \mathrm{mg} / 100 \mathrm{kcal}$, (using $670 \mathrm{kcal} / \mathrm{L}$ as a conversion factor); this requirements can be expressed as 33508040mg/L for LA and 335-667 mg/L for ALA. In addition, other organizations around the world [142] also specify adequate intake levels for DHA of $20 \mathrm{mg} / \mathrm{kg} /$ day for infants 0-5 months and up to $100 \mathrm{mg} /$ day for infants 7- 24 months of DHA.

Table 30. Human milk FA concentrations (mg/L). Taken from Yuhas et al.[104]

\begin{tabular}{|c|c|c|c|c|c|c|c|c|c|}
\hline \multirow{2}{*}{$\begin{array}{l}\text { FA } \\
\text { Country }\end{array}$} & \multirow[b]{2}{*}{$\mathrm{n}$} & \multicolumn{2}{|l|}{ LA } & \multicolumn{2}{|l|}{ ALA } & \multicolumn{2}{|l|}{$\mathrm{AA}$} & \multicolumn{2}{|l|}{ DHA } \\
\hline & & Mean & SE & Mean & SE & Mean & SE & Mean & SE \\
\hline Australia & 48 & 4233.1 & 217.43 & 348.5 & 18.28 & 148.7 & 6.90 & 93.4 & 14.41 \\
\hline Canada & 48 & 4658.6 & 250.15 & 501.7 & 28.17 & 151.3 & 8.68 & 73.3 & 7.52 \\
\hline Chile & 50 & 5073.7 & 401.69 & 320.0 & 28.73 & 116.4 & 8.36 & 116.6 & 8.25 \\
\hline China & 50 & 6299.3 & 369.74 & 863.5 & 58.32 & 207.8 & 12.05 & 149.8 & 10.50 \\
\hline Japan & 51 & 4546.8 & 263.47 & 481.4 & 36.76 & 141.9 & 8.24 & 365.4 & 39.78 \\
\hline Mexico & 46 & 5415.5 & 322.03 & 353.0 & 28.01 & 140.7 & 7.93 & 86.0 & 10.17 \\
\hline Philippines & 54 & 3524.8 & 186.81 & 195.3 & 15.45 & 171.5 & 8.18 & 337.7 & 35.06 \\
\hline United Kingdom & 44 & 4034.1 & 260.88 & 480.2 & 35.92 & 141.5 & 8.95 & 95.6 & 9.12 \\
\hline United States & 49 & 4421.2 & 273.22 & 316.3 & 25.47 & 132.9 & 8.66 & 48.8 & 5.16 \\
\hline Global mean & 440 & 4683.9 & 102.70 & 427.1 & 13.88 & 150.7 & 3.13 & 156.2 & 8.68 \\
\hline
\end{tabular}

Table 29 presents the concentrations in $\mathrm{mg} / \mathrm{L}$ of FA in 74 Peruvian women in mature milk. The mean levels reported here for the essential FA and LC-PUFAs meet all the international guidelines for adequate intakes of n-6 and n-3 PUFAS recommended for infants during the first months. Moreover, when comparing quantities from Table 29 with the quantities presented in Table 30 for n-6 and n-3 PUFAs from different countries, 
we can conclude that dietary supply of essential FA is sufficient in the Peruvian population studied during the first month of lactation. 


\section{CHAPTER IV: Carotenoids in human milk}

Human milk is the sole source of nourishment for most infants during the first months of life. Essential fat soluble nutrients including carotenoids that cannot synthesize by humans are transported in the emulsified fat globules present in the mother's milk. Both pro-vitamin A and non pro-vitamin A carotenoids act as antioxidants and provide protection against chronic diseases $[76,143]$. The fatty acid composition of triglycerides and in milk changes during lactation. Colostrum, the secretion produced by the mother's mammary glands during the first days after giving birth [144] is rich in essential nutrients and antibodies and the yellow coloration has been suggested to be due to the high concentration of carotenoids derived from the mother's stores [145].

As described in the previous chapters, it has been suggested that lutein and zeaxanthin as well as the essential polyunsaturated fatty acids (PUFAS) such as linoleic acid and $\alpha$-linolec acid are important in early vision and neural development. Long chain n-3 and n-6 PUFAS, such as docosahexanoic acid (DHA) and arachidonic acid (AA) are known to be important in visual function and neural development [39, 99]. All of these lipophilic nutrients are acquired by the new born through lactation, and the carotenoids are solubilized in the fat globules present in human milk. The mechanism of transport of carotenoids from the mother's blood stream into milk remains incompletely understood. It has been hypothesized that a selective mechanism may be responsible for the transport of carotenoids into human milk. Specifically, because carotenoids are lipophilic in nature and co-transported with lipids, it may be that their transport is in association with specific fatty acids. Little information is available about carotenoid transport and so we have 
analyzed carotenoid levels in human milk during the first month of lactation in an effort to improve our understanding $[135,146,147]$. Most of the previously published studies reporting measurement of carotenoid levels in human milk [148-150] have focused on mature milk obtained from the later stages in lactation. Our goal here was to increase the data base on carotenoid levels in human milk during the first month with the goal of improving our understanding of the uptake and transport of carotenoids. In Chapter 3, the amounts of fatty acids measured in the lipid fraction of breast milk samples from 74 Peruvian new mothers were reported and discussed. In this Chapter, our measurements of the carotenoids found in the milk of these women are presented and correlated with the results of our analysis of the individual fatty acids in human milk during the first month of lactation.

\subsection{Research Methods}

\subsubsection{Study population}

The participants in this study were 74 Peruvian mothers having a mean age of 26.2 years and an average BMI of $27.97 \pm 3.98$ (BMI was available for 70 participants). This was the first pregnancy for all participants and none reported chronic health concerns. All of the deliveries occurred after the full 37 weeks of gestation. All volunteers were informed about the study and signed an IRB consent document.

\subsubsection{Chemicals}

Ethyl alcohol USP grade, potassium hydroxide, butylated hydroxytoluene, USP grade (BHT) were all purchased from Sigma-Aldrich or Fisher Scientific. HPLC grade 
hexane, dichloromethane, methanol, and acetonitrile were purchased from SigmaAldrich. Lutein and zeaxanthin standards were a gift from Industrial Organica S.A. $\beta$ carotene standard was purchased from Sigma-Aldrich. Methyl pentyl lutein (MPL) and $\beta$ cryptoxanthin standards were prepared and characterized by methods described in Chapter 1.

\subsubsection{Instruments}

A Varian ProStar HPLC system coupled to a ProStar 335 photodiode array UVvisible detection system was used for carotenoid analysis. A Phenomenex 250 x $4.60 \mathrm{~mm}$ ODS ultracarb $3 \mu \mathrm{m}$ column (20) of was used for all primary carotenoid separations, with a mobile phase consisting of acetonitrile: methanol: triethylamine (85\%: $15 \%: 0.1 \% \mathrm{v} / \mathrm{v})$ and a flow rate of $1 \mathrm{~mL} / \mathrm{min}$ with a detection at $450 \mathrm{~nm}$. Chromatographic data were analyzed using the Varian software package.

\subsubsection{Carotenoid analysis in milk samples}

The extraction of the carotenoids from milk samples was carried out following the procedure used by Jackson et al. [151]. Milk samples were thawed at room temperature and shaken gently to homogenize the sample prior to pipetting an aliquot for analysis. 2 $\mathrm{mL}$ of milk and $200 \mu \mathrm{g}$ of MPL standard were placed in a Teflon screw cap Pyrex tube $(15 \times 125 \mathrm{~mm})$. Saponification of samples was carried out by adding $1.5 \mathrm{~mL}$ of $50 \%(\mathrm{w} / \mathrm{v})$ potassium hydroxide and $2.5 \mathrm{~mL}$ absolute ethanol to the tube. After vortexing the tube, the contents was placed in a water bath at $37-38^{\circ} \mathrm{C}$ for 2 hours and the tubes were occasionally stirred. Carotenoids were extracted three times from the mixture using $3 \mathrm{~mL}$ aliquots of hexane containing $0.025 \%(\mathrm{w} / \mathrm{v})$ BHT. The extracts were combined and 
evaporated under nitrogen. The resulting residue was re-dissolved into $80 \mu \mathrm{L}$ of ethanol prior to HPLC analysis.

\subsubsection{Chromatographic separation analysis}

Carotenoid levels in all samples were analyzed by reversed phase HPLC, using a Phenomenex $250 \times 4.60 \mathrm{~mm}$ ODS ultracarb $3 \mu \mathrm{m}$ column (20). The mobile phase used for the separation was a mixture of acetonitrile/methanol/TEA ( $85 \%: 15 \%: 0.1 \% \mathrm{v} / \mathrm{v})$. The flow rate was $1 \mathrm{~mL} /$ minute and the sample was run at $25^{\circ} \mathrm{C}$ for 90 minutes. Quantitation of the individual components was carried out by comparing the peak areas in chromatograms to that of the added internal standard.

\subsubsection{Statistical Analysis}

Data were analyzed using Statistical Packages for Social Sciences version 21.0 (SPSS In c. Chicago, IL, USA). For comparison a two-way ANOVA test was conducted for a group of 26 subjects that donated milk samples during the three consecutive weeks of the study.

\subsection{Results}

We have analyzed the carotenoid content in a total of 133 human milk samples. A typical chromatogram obtained for a milk sample is shown in Figure 41. Analytical results of the carotenoid concentrations in maternal milk have been collected and are found in Tables 31- 33. The results for samples obtained during each week post-partum are displayed separately: Week 2 (W2), Week 3 (W3), and Week 4 (W4).

Table 31. Carotenoid concentrations in human milk during the 2 nd week of lactation (transitional milk, W2). 


\begin{tabular}{|c|c|c|c|c|c|c|c|c|c|}
\hline \multicolumn{10}{|c|}{ Transitional milk (week 2 post-partum) ( $\boldsymbol{9 m o l} / \mathbf{L})$} \\
\hline Subject & Lutein & Zea & $\boldsymbol{\beta c r y}$ & $\boldsymbol{\beta c a r}$ & Subject & Lutein & Zea & $\boldsymbol{\beta c r y}$ & $\boldsymbol{\beta c a r}$ \\
\hline $\mathbf{2 7 0 1}$ & 0.24 & 0.11 & 0.09 & 0.06 & $\mathbf{4 7 0 2}$ & 0.14 & 0.06 & 0.21 & 0.08 \\
$\mathbf{2 7 0 2}$ & 0.07 & 0.03 & 0.06 & $* * *$ & $\mathbf{4 7 0 3}$ & 0.03 & 0.01 & 0.03 & 0.04 \\
$\mathbf{2 7 0 3}$ & 0.05 & 0.02 & $* * *$ & $* * *$ & $\mathbf{4 7 0 5}$ & 0.11 & 0.04 & 0.12 & 0.05 \\
$\mathbf{2 7 0 4}$ & 0.09 & 0.02 & 0.07 & $* * *$ & $\mathbf{4 7 0 6}$ & 0.05 & 0.02 & 0.03 & $* * *$ \\
$\mathbf{2 7 0 5}$ & 0.11 & 0.06 & 0.05 & 0.03 & $\mathbf{4 7 0 8}$ & 0.04 & 0.02 & 0.02 & $* * *$ \\
$\mathbf{2 7 0 6}$ & 0.05 & 0.03 & 0.10 & 0.02 & $\mathbf{4 7 0 9}$ & 0.05 & 0.02 & 0.06 & 0.07 \\
$\mathbf{2 7 0 7}$ & 0.11 & 0.03 & 0.08 & 0.06 & $\mathbf{4 7 1 0}$ & 0.04 & 0.02 & 0.04 & 0.05 \\
$\mathbf{2 7 0 8}$ & 0.06 & 0.02 & 0.03 & 0.06 & $\mathbf{4 7 1 2}$ & 0.06 & 0.04 & 0.10 & 0.03 \\
$\mathbf{2 7 0 9}$ & 0.02 & 0.01 & 0.05 & $* * *$ & $\mathbf{4 7 1 3}$ & 0.05 & 0.03 & 0.18 & 0.02 \\
$\mathbf{2 7 1 0}$ & 0.04 & 0.02 & 0.02 & $* * *$ & $\mathbf{4 7 1 4}$ & 0.04 & 0.02 & 0.03 & 0.02 \\
$\mathbf{2 7 1 1}$ & 0.06 & 0.03 & 0.05 & $* * *$ & $\mathbf{4 7 1 5}$ & 0.05 & 0.04 & 0.10 & 0.04 \\
$\mathbf{2 7 1 3}$ & 0.51 & 0.24 & 0.72 & 0.17 & $\mathbf{4 7 1 6}$ & 0.05 & 0.02 & 0.10 & $* * *$ \\
$\mathbf{2 7 1 4}$ & 0.03 & 0.02 & 0.02 & 0.03 & $\mathbf{4 7 1 7}$ & 0.04 & 0.02 & 0.05 & 0.03 \\
$\mathbf{2 7 1 5}$ & 0.05 & 0.02 & 0.17 & 0.02 & $\mathbf{4 7 1 8}$ & 0.02 & 0.01 & 0.02 & 0.03 \\
$\mathbf{4 7 0 1}$ & 0.15 & 0.08 & 0.06 & $* * *$ & $\mathbf{4 7 1 9}$ & 0.06 & 0.03 & 0.04 & 0.08 \\
\hline
\end{tabular}

$* * *$ Not detected

Table 32. Carotenoid concentrations in human milk during the 3rd week of lactation (mature milk, W3).

\begin{tabular}{|c|c|c|c|c|c|c|c|c|c|}
\hline \multicolumn{10}{|c|}{ Mature milk (week 3 post-partum) $(\boldsymbol{\mu m o l} / \mathbf{L})$} \\
\hline Subject & Lutein & Zea & $\boldsymbol{\beta c r y}$ & $\boldsymbol{\beta c a r}$ & Subject & Lutein & Zea & $\boldsymbol{\beta c r y}$ & $\boldsymbol{\beta}$ car \\
\hline $\mathbf{2 7 0 1}$ & 0.06 & 0.04 & 0.02 & 0.02 & $\mathbf{4 7 0 2}$ & 0.04 & 0.01 & 0.01 & 0.05 \\
$\mathbf{2 7 0 2}$ & 0.04 & 0.02 & $* * *$ & $* * *$ & $\mathbf{4 7 0 3}$ & 0.03 & 0.01 & 0.01 & 0.03 \\
$\mathbf{2 7 0 3}$ & 0.08 & 0.02 & $* * *$ & $* * *$ & $\mathbf{4 7 0 5}$ & 0.03 & 0.02 & 0.03 & 0.03 \\
$\mathbf{2 7 0 4}$ & 0.04 & 0.02 & $* * *$ & $* * *$ & $\mathbf{4 7 0 6}$ & 0.01 & 0.01 & 0.02 & $* * *$ \\
$\mathbf{2 7 0 5}$ & 0.07 & 0.03 & 0.02 & $* * *$ & $\mathbf{4 7 0 8}$ & 0.04 & 0.02 & 0.02 & $* * *$ \\
$\mathbf{2 7 0 6}$ & 0.05 & 0.02 & 0.04 & 0.04 & $\mathbf{4 7 0 9}$ & 0.03 & 0.01 & 0.03 & 0.03 \\
$\mathbf{2 7 0 7}$ & 0.05 & 0.02 & 0.11 & 0.03 & $\mathbf{4 7 1 0}$ & 0.02 & 0.01 & 0.02 & 0.03 \\
$\mathbf{2 7 0 8}$ & 0.04 & 0.01 & 0.02 & $* * *$ & $\mathbf{4 7 1 2}$ & 0.13 & 0.04 & 0.14 & 0.09 \\
$\mathbf{2 7 0 9}$ & 0.01 & 0.01 & 0.06 & $* * *$ & $\mathbf{4 7 1 3}$ & 0.06 & 0.03 & 0.21 & 0.05 \\
$\mathbf{2 7 1 0}$ & 0.04 & 0.01 & $* * *$ & $* * *$ & $\mathbf{4 7 1 4}$ & 0.09 & 0.05 & 0.06 & 0.05 \\
$\mathbf{2 7 1 1}$ & 0.04 & 0.02 & 0.03 & $* * *$ & $\mathbf{4 7 1 5}$ & 0.03 & 0.02 & 0.04 & 0.03 \\
$\mathbf{2 7 1 3}$ & 0.08 & 0.03 & 0.13 & 0.10 & $\mathbf{4 7 1 6}$ & 0.03 & 0.02 & 0.14 & 0.02 \\
$\mathbf{2 7 1 4}$ & 0.02 & 0.02 & 0.02 & $* * *$ & $\mathbf{4 7 1 7}$ & 0.01 & 0.01 & 0.07 & $* * *$ \\
$\mathbf{2 7 1 5}$ & 0.05 & 0.02 & 0.30 & 0.00 & $\mathbf{4 7 1 9}$ & 0.04 & 0.01 & 0.03 & 0.04 \\
\hline
\end{tabular}

$* * *$ Not detected 


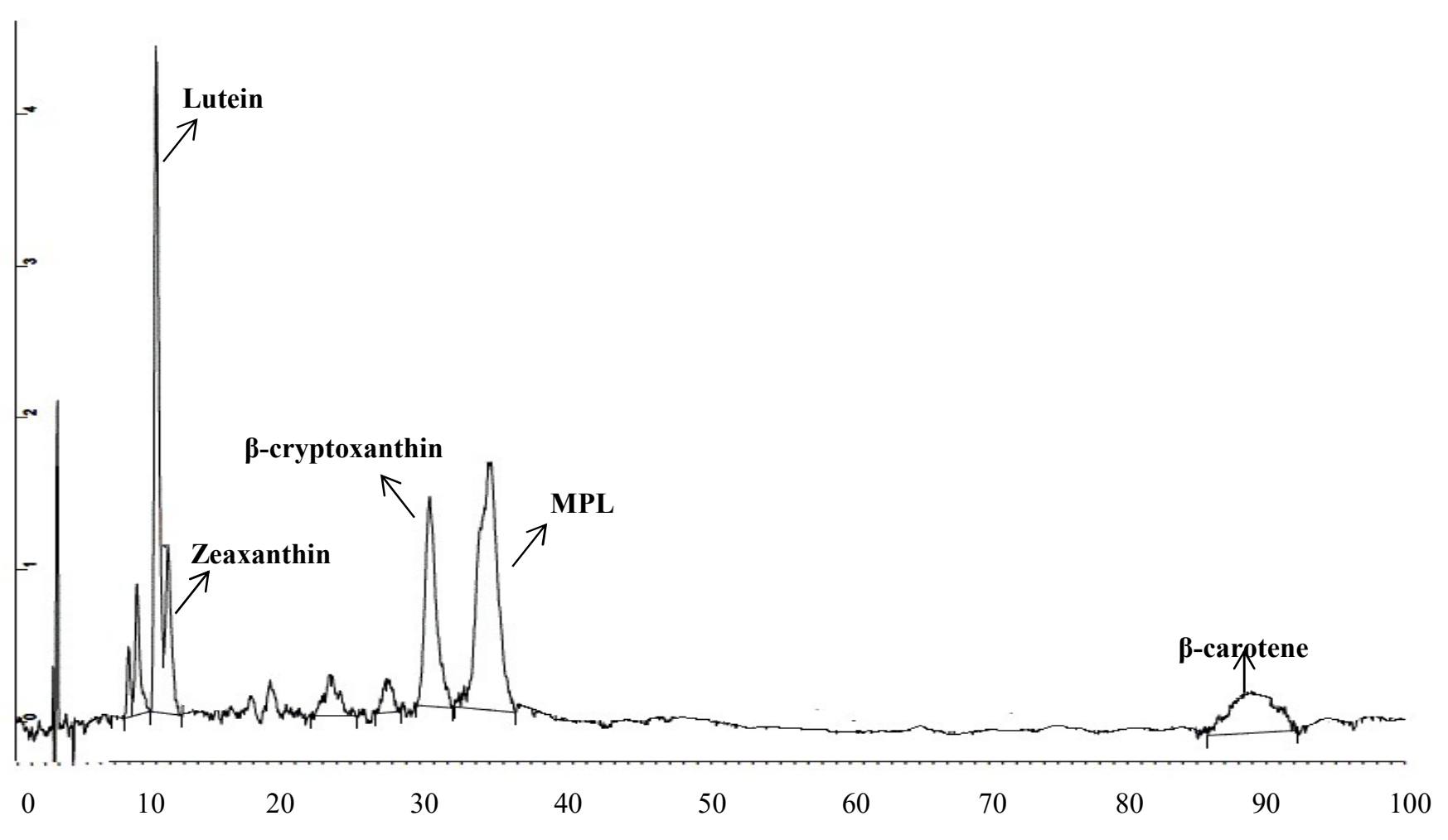

Figure 41. Typical reversed-phase HPLC chromatogram of the carotenoid analysis of a sample of human milk. 
Table 33. Carotenoid concentrations in human milk during the 4th week of lactation (mature milk, W4).

\begin{tabular}{|c|c|c|c|c|c|c|c|c|c|}
\hline \multicolumn{10}{|c|}{ Mature milk (week 4 post-partum) $(\mu \mathrm{mol} / \mathrm{L})$} \\
\hline Subject & Lutein & Zea & Bcry & Bcar & Subject & Lutein & Zea & 及cry & Bcar \\
\hline 2701 & 0.08 & 0.02 & 0.03 & $* * *$ & 2723 & 0.04 & 0.01 & 0.02 & 0.02 \\
\hline 2702 & 0.04 & 0.02 & 0.02 & $* * *$ & 2724 & 0.02 & 0.01 & 0.03 & $* * *$ \\
\hline 2703 & 0.04 & 0.01 & 0.00 & $* * *$ & 2725 & 0.05 & 0.02 & 0.05 & 0.02 \\
\hline 2704 & 0.02 & 0.00 & $* * *$ & $* * *$ & 2726 & 0.04 & 0.01 & 0.05 & $* * *$ \\
\hline 2705 & 0.07 & 0.02 & 0.05 & 0.02 & 2727 & 0.07 & 0.02 & 0.10 & $* * *$ \\
\hline 2706 & 0.02 & 0.01 & 0.05 & 0.03 & 2728 & 0.02 & 0.01 & 0.09 & $* * *$ \\
\hline 2707 & 0.09 & 0.03 & 0.04 & $* * *$ & 2729 & 0.03 & 0.01 & 0.02 & $* * *$ \\
\hline 2708 & 0.04 & 0.02 & 0.03 & 0.05 & 2730 & 0.18 & 0.07 & 0.09 & $* * *$ \\
\hline 2709 & 0.01 & 0.01 & 0.04 & $* * *$ & 2732 & 0.02 & 0.01 & 0.01 & $* * *$ \\
\hline 2710 & 0.06 & 0.02 & 0.03 & 0.02 & 2733 & 0.03 & 0.01 & 0.01 & $* * *$ \\
\hline 2712 & 0.04 & 0.01 & 0.02 & 0.03 & 2735 & 0.04 & 0.01 & 0.08 & $* * *$ \\
\hline 2713 & 0.07 & 0.02 & 0.13 & 0.10 & 2736 & 0.10 & 0.04 & 0.05 & $* * *$ \\
\hline 2714 & 0.02 & 0.01 & 0.03 & 0.06 & 2737 & 0.06 & 0.02 & 0.07 & 0.04 \\
\hline 2715 & 0.04 & 0.02 & 0.09 & $* * *$ & 2739 & 0.12 & 0.03 & 0.15 & 0.06 \\
\hline 4701 & 0.06 & 0.03 & 0.03 & 0.02 & 2740 & 0.08 & 0.02 & 0.18 & $* * *$ \\
\hline 4702 & 0.06 & 0.03 & 0.06 & $* * *$ & 2741 & 0.05 & 0.02 & 0.07 & 0.03 \\
\hline 4704 & 0.03 & 0.01 & 0.02 & 0.02 & 2742 & 0.03 & 0.01 & 0.04 & 0.01 \\
\hline 4705 & 0.05 & 0.02 & 0.02 & $* * *$ & 2743 & 0.05 & 0.02 & 0.06 & $* * *$ \\
\hline 4706 & 0.02 & 0.01 & 0.02 & $* * *$ & 4720 & 0.07 & 0.03 & 0.05 & $* * *$ \\
\hline 4708 & 0.04 & 0.02 & 0.02 & 0.03 & 4721 & 0.02 & 0.01 & 0.14 & $* * *$ \\
\hline 4709 & 0.05 & 0.02 & 0.06 & 0.07 & 4722 & 0.02 & 0.01 & 0.03 & $* * *$ \\
\hline 4710 & 0.05 & 0.01 & 0.02 & $* * *$ & 4723 & 0.06 & 0.03 & 0.09 & 0.04 \\
\hline 4712 & 0.08 & 0.04 & 0.12 & 0.08 & 4724 & 0.03 & 0.02 & 0.06 & $* * *$ \\
\hline 4713 & 0.09 & 0.05 & 0.12 & 0.03 & 4725 & 0.11 & 0.02 & 0.12 & 0.03 \\
\hline 4714 & 0.04 & 0.01 & 0.03 & 0.03 & 4726 & 0.12 & 0.04 & 0.12 & $* * *$ \\
\hline 4715 & 0.05 & 0.02 & 0.07 & 0.06 & 4727 & 0.06 & 0.02 & 0.12 & $* * *$ \\
\hline 4716 & 0.04 & 0.02 & 0.13 & 0.02 & 4728 & 0.05 & 0.02 & 0.07 & 0.02 \\
\hline 4717 & 0.02 & 0.02 & 0.06 & 0.05 & 4729 & 0.04 & 0.02 & 0.09 & $* * *$ \\
\hline 4718 & 0.03 & 0.01 & 0.02 & 0.03 & 4730 & 0.09 & 0.03 & $* * *$ & $* * *$ \\
\hline 4719 & 0.03 & 0.01 & 0.02 & 0.03 & 4731 & 0.05 & 0.03 & 0.07 & $* * *$ \\
\hline 2716 & 0.03 & 0.01 & 0.07 & 0.02 & 4732 & 0.03 & 0.02 & 0.02 & $* * *$ \\
\hline 2717 & 0.06 & 0.02 & 0.03 & $* * *$ & 4733 & 0.02 & 0.01 & 0.02 & $* * *$ \\
\hline 2718 & 0.03 & 0.02 & 0.05 & $* * *$ & 4736 & 0.08 & 0.02 & 0.03 & $* * *$ \\
\hline 2719 & 0.03 & 0.02 & 0.07 & 0.02 & 4738 & 0.03 & 0.02 & 0.05 & $* * *$ \\
\hline 2720 & 0.05 & 0.02 & 0.04 & $* * *$ & 4739 & 0.03 & 0.02 & 0.10 & $* * *$ \\
\hline 2721 & 0.02 & 0.01 & 0.04 & 0.01 & 4740 & 0.02 & 0.01 & 0.02 & $* * *$ \\
\hline 2722 & 0.02 & 0.01 & 0.06 & $* * *$ & 4741 & 0.07 & 0.03 & 0.07 & $* * *$ \\
\hline
\end{tabular}

*** Not detected 
Table 34. Carotenoid concentrations in maternal serum and milk during the 4 th week (W4) after delivery.

\begin{tabular}{|c|c|cc|cc|c|}
\cline { 3 - 7 } \multicolumn{1}{c|}{} & \multicolumn{2}{c|}{ Serum $(\boldsymbol{\mu m o l} / \mathbf{L})$} & \multicolumn{2}{c|}{ Milk W4 $(\boldsymbol{\mu m o l} / \mathbf{L})$} & \multicolumn{1}{c|}{} \\
\cline { 2 - 7 } \multicolumn{1}{c|}{} & $\mathbf{N}$ & Range & $\begin{array}{c}\text { Concentration } \\
\text { Mean } \pm \text { S.D. }\end{array}$ & Range & $\begin{array}{c}\text { Concentration } \\
\text { Mean } \pm \text { S.D. }\end{array}$ & r \\
\hline Lutein & 73 & $0.12-0.69$ & $0.27 \pm 0.12$ & $0.01-0.18$ & $0.05 \pm 0.03$ & $0.429^{*}$ \\
Zea & 73 & $0.05-0.27$ & $0.11 \pm 0.04$ & $0.01-0.07$ & $0.02 \pm 0.01$ & $0.361^{*}$ \\
$\boldsymbol{\beta}$-cry & 71 & $0.07-1.83$ & $0.40 \pm 0.32$ & $0.004-0.18$ & $0.06 \pm 0.04$ & $0.592^{*}$ \\
L/Z & 26 & $0.07-1.10$ & $0.34 \pm 0.23$ & $0.02-0.08$ & $0.04 \pm 0.02$ & 0.177 \\
\hline
\end{tabular}

$* \mathrm{p}<0.003$

Levels of lutein, zeaxanthin, $\beta$-cryptoxanthin, and $\beta$-carotene in maternal milk during the three weeks studied were compared to document any longitudinal changes. Strong correlations $(\mathrm{p}<0.001)$ between lutein and zeaxanthin were observed for all three weeks, but no correlation of carotenoid levels with $\%$ of fatty acids in milk was observed.

Values for the concentration of lutein, zeaxanthin, $\beta$-cryptoxanthin, and $\beta$ carotene in maternal milk were compared with those obtained from matched serum samples during week 4. Positive correlations $(p<0.003)$ were observed for lutein, zeaxanthin, and $\beta$-cryptoxanthin concentrations having $\mathrm{r}$ values of $0.429,0.361$, and 0.592, respectively. Lutein and zeaxanthin concentrations were 5.4-5.5 times lower and pro-vitamin A carotenoids were more than 6.6 times lower in milk than in maternal serum. The lutein:zeaxanthin ratios were also positively correlated positively between milk and serum $(r=0.575, \mathrm{p}<0.0001)$, see Table 34 .

A comparison of carotenoid concentrations in maternal milk during the fourth week of study is presented in Table 35 for the same group of subjects. A two-way ANOVA test run for the data shows that the levels of lutein and zeaxanthin are 
significantly different between transitional milk (W2) and mature milk (W3 and W4). Pro-vitamin A carotenoids, $\beta$-cryptoxanthin and $\beta$-carotene showed a different pattern. Only $\beta$-cryptoxanthin was significantly decreased in concentration between transitional milk (W2) and mature milk during the last week (W4), while $\beta$-carotene did not show any significant change in concentration during the first month. In the same manner as seen in chapter 3, \% total fatty acids in milk did not change. A paired t-test run for lutein and $\beta$-cryptoxanthin levels for each week (W2 $\mathrm{p}=0.28, \mathrm{~W} 3 \mathrm{p}=0.11, \mathrm{~W} 4 \mathrm{p}=0.62$ ), showed that the concentration levels are not statistically different between these two carotenoids during the same week.

For the same subjects lutein, $\beta$-cryptoxanthin, and $\beta$-carotene levels are each strongly correlated between transitional milk (W2) and mature milk (W4), and between W3 and W4 for mature milk. In the same comparison, no correlation was observed for zeaxanthin. No correlation of the carotenoids with total percent of fatty acids in milk was observed during the time of lactation.

Table 35. Longitudinal, subject by subject comparison of carotenoid concentrations and total fatty acid content between weeks. Values reported are mean concentrations (mean \pm SEM) in $\mu \mathrm{mol} / \mathrm{L}$.

\begin{tabular}{|c|c|cc|cc|cc|}
\cline { 2 - 8 } \multicolumn{1}{c|}{} & \multicolumn{2}{c|}{ Week 2 } & \multicolumn{2}{c|}{ Week 3 } & \multicolumn{2}{c|}{ Week 4 } \\
\cline { 2 - 8 } \multicolumn{1}{c|}{} & $\mathbf{N}$ & Range & Conc. & Range & Conc. & Range & Conc. \\
\hline Lutein & 26 & $0.20-0.51$ & $0.09 \pm 0.02$ & $0.10-0.13$ & $0.05 \pm 0.01^{\mathbf{a}}$ & $0.10-0.09$ & $0.05 \pm 0.01^{\mathbf{a}}$ \\
Zea & 26 & $0.10-0.24$ & $0.04 \pm 0.01$ & $0.10-0.50$ & $0.02 \pm 0.00^{\mathbf{a}}$ & $0.01-0.50$ & $0.02 \pm 0.00^{\mathbf{a}}$ \\
$\boldsymbol{\beta}$-cry & 22 & $0.02-0.72$ & $0.10 \pm 0.03$ & $0.01-0.30$ & $0.07 \pm 0.02$ & $0.00-0.13$ & $0.05 \pm 0.02^{\mathbf{a}}$ \\
$\boldsymbol{\beta}$-car & 15 & $0.02-0.17$ & $0.05 \pm 0.01$ & $0.00-0.10$ & $0.05 \pm 0.01$ & $0.02-0.10$ & $0.05 \pm 0.01$ \\
L/Z & 26 & $1.25-4.23$ & $2.32 \pm 0.12$ & $1.27-3.72$ & $2.28 \pm 0.13$ & $1.40-4.34$ & $2.53 \pm 0.12$ \\
\% FA & 26 & $1.23-5.58$ & $3.40 \pm 0.20$ & $0.78-5.28$ & $3.26 \pm 0.22$ & $1.20-6.43$ & $3.26 \pm 0.28$ \\
\hline
\end{tabular}

${ }^{\mathrm{a}}$ Significantly different $(\mathrm{p}<0.05)$ than transitional milk $\left(2^{\text {nd }}\right.$ week, W2). 
Because the carotenoid level in serum has previously been reported to be correlated with maternal BMI carotenoid concentrations in mature milk during week 4 were grouped according to maternal BMI and are shown in Table 36. The subjects were split in three groups: normal $(\mathrm{BMI}=18.5-24.9)$, overweight $(\mathrm{BMI}=25.0-29.9)$, and obese $(\mathrm{BMI}>30.0)$. There was no difference in the levels of lutein and zeaxanthin in milk between these groups but pro-vitamin A carotenoids levels in maternal milk were different between the three groups. Pro-vitamin A carotenoid concentrations in milk decrease as BMI increased. $\beta$-cryptoxanthin, a pro-vitamin A carotenoid, showed a statistically significant decrease but only between the normal and obese groups.

Table 36. Carotenoid concentrations (mean \pm st.dev., $\mu \mathrm{mol} / \mathrm{L}$ ) in mature maternal milk (W4) grouped according to the subject's body mass index.

\begin{tabular}{|c|c|c|c|}
\hline & Normal & Overweight & Obese \\
\hline $\mathbf{N}$ & 17 & 32 & 19 \\
BMI & $23.18 \pm 1.36^{\mathrm{a}}$ & $27.49 \pm 1.35$ & $32.85 \pm 2.87$ \\
Lutein & $0.05 \pm 0.03$ & $0.05 \pm 0.03$ & $0.05 \pm 0.02$ \\
Zeaxanthin & $0.02 \pm 0.01$ & $0.02 \pm 0.02$ & $0.02 \pm 0.01$ \\
$\boldsymbol{\beta}$-cryptoxanthin & $0.08 \pm 0.04^{\mathbf{b}}$ & $0.06 \pm 0.04$ & $0.05 \pm 0.03$ \\
$\boldsymbol{\beta}$-carotene & $0.05 \pm 0.03$ & $0.04 \pm 0.02$ & $0.03 \pm 0.01$ \\
\% total FA & $3.72 \pm 1.64$ & $3.76 \pm 1.13$ & $(\mathrm{~N}=9)$ \\
\hline
\end{tabular}

a Significantly different $(\mathrm{p}<0.001)$ than overweight and obese group.

${ }^{b}$ Significantly different $(\mathrm{p}<0.05)$ than obese group.

Pearson correlation coefficients obtained for a subject by subject correlation of the individual lutein, zeaxanthin, $\beta$-cryptoxanthin, and $\beta$-carotene concentrations in human milk with the concentrations of the individual fatty acids in all three weeks 
reported in Chapter 3 were determined. Tables 37 and 38 show those results that had a statistically significant correlation between concentrations any of the FAs and any of the carotenoids studied in milk.

Table 37. Pearson correlation coefficients obtained from the subject by subject comparison of carotenoid concentrations and individual fatty acid concentrations in transitional milk (W2).

\begin{tabular}{|c|c|c|c|c|c|c|c|c|}
\hline & \multicolumn{8}{|c|}{ Week 2} \\
\hline & $\mathbf{N}$ & C14:0 & C16:0 & C20:1n-9 & C24:1n-9 & $C 20: 2 n-6$ & C18:2n-6 & C18:3n-3 \\
\hline Lutein & 30 & $\begin{array}{l}r=0.486 \\
p=0.006\end{array}$ & $\begin{array}{l}r=0.375 \\
p=0.04\end{array}$ & $\begin{array}{l}r=0.459 \\
p=0.01\end{array}$ & $\begin{array}{l}r=0.366^{*} \\
p=0.07\end{array}$ & $\begin{array}{l}\mathrm{r}=0.331^{*} \\
\mathrm{p}=0.07\end{array}$ & $\begin{array}{l}\mathrm{r}=-0.432 \\
\mathrm{p}=0.02\end{array}$ & $\begin{array}{l}r=-0.435 \\
p=0.02\end{array}$ \\
\hline Zea & 30 & $\begin{array}{l}r=0.468 \\
p=0.009\end{array}$ & $\begin{array}{l}r=0.381 \\
p=0.04\end{array}$ & $\begin{array}{l}r=0.468 \\
p=0.009\end{array}$ & $\begin{array}{l}r=0.377 \\
p=0.04\end{array}$ & $\begin{array}{l}\mathrm{r}=0.341 * \\
\mathrm{p}=0.06\end{array}$ & $\begin{array}{l}\mathrm{r}=-0.423 \\
\mathrm{p}=0.02\end{array}$ & $\begin{array}{l}r=-0.418 \\
p=0.02\end{array}$ \\
\hline$\beta$-cry & 29 & $\begin{array}{l}r=0.483 \\
p=0.008\end{array}$ & N.S.C. & N.S.C. & $\begin{array}{l}r=0.388 \\
p=0.04\end{array}$ & $\begin{array}{l}\mathrm{r}=0.477 \\
\mathrm{p}=0.009\end{array}$ & $\begin{array}{l}r=-0.384 \\
p=0.04\end{array}$ & $\begin{array}{l}r=-0.312 \\
p=0.099\end{array}$ \\
\hline$\beta$-car & 20 & $\begin{array}{l}r=0.453 \\
p=0.04\end{array}$ & N.S.C. & $\begin{array}{l}r=0.436 \\
p=0.05\end{array}$ & $\begin{array}{l}\mathrm{r}=0.432^{*} \\
\mathrm{p}=0.06\end{array}$ & N.S.C. & $\begin{array}{l}r=-0.489 \\
p=0.03\end{array}$ & $\begin{array}{l}r=-0.569 \\
p=0.009\end{array}$ \\
\hline
\end{tabular}

N.S.C $=$ no significant correlation was observed.

* correlation approaches significance.

A comparison of SFA levels with carotenoids levels reveals a positive correlation for all carotenoids reported here in transitional milk (W2) with myristic acid (C14:0). Only lutein and zeaxanthin showed a positive correlation with palmitic acid (C16:0) in transitional milk (W2). In a comparison of MUFAs with carotenoid concentrations, lutein, zeaxanthin, and $\beta$-carotene were all found to be positively correlated with eicosenoic acid (C20:1n-9) in transitional milk (W2). Analysis also shows that all carotenoids except $\beta$-carotene are significantly and positively correlated with nervoic acid (C24:1n-9) in transitional milk (W2). Although $\beta$-carotene did not reach a significant statistical level it was trends positively with this fatty acid in transitional milk. 
Comparison of the levels of individual PUFAs in transitional milk (W2), reveals that eicosadienoic acid $(\mathrm{C} 20: 2 \mathrm{n}-6)$ is positive correlated with lutein, zeaxanthin and $\beta$ cryptoxanthin, but zeaxanthin, although positively correlated does not reach a statistically significant level. The essential fatty acids linoleic (C18:2n-6) and $\alpha$-linoleic acid (C18:3n-3) were found to be significantly inversely correlated with all carotenoids measured in this study in transitional milk (W2).

Table 38. Pearson correlation coefficients obtained from the subject by subject comparision of carotenoid concentrations and individual fatty acid concentrations in in mature milk (W3 and W4).

\begin{tabular}{|c|c|c|c|c|c|c|c|c|}
\hline & \multicolumn{4}{|c|}{ Week 3} & \multicolumn{4}{|c|}{ Week 4} \\
\hline & $\mathbf{N}$ & C14:0 & C18:1n-9 & C20:0 & $\mathbf{N}$ & C20:1n-9 & C20:2n-6 & C24:0 \\
\hline Lutein & 28 & $\begin{array}{l}\mathrm{r}=0.336 \\
\mathrm{p}=0.008\end{array}$ & $\begin{array}{l}\mathrm{r}=-0.370 \\
\mathrm{p}=0.05\end{array}$ & N.S.C. & 73 & N.S.C. & N.S.C. & N.S.C. \\
\hline Zea & 28 & N.S.C. & N.S.C. & N.S.C. & 73 & $\begin{array}{l}r=0.209^{*} \\
\mathrm{p}=0.08\end{array}$ & N.S.C. & $\begin{array}{l}r=0.258 \\
p=0.03\end{array}$ \\
\hline$\beta$-cry & 24 & N.S.C. & N.S.C. & $\begin{array}{l}r=-0.755 \\
p=0.000\end{array}$ & 71 & $\begin{array}{l}r=0.291 \\
p=0.01\end{array}$ & $\begin{array}{l}r=0.316 \\
p=0.007\end{array}$ & N.S.C. \\
\hline$\beta$-car & 16 & $\begin{array}{l}r=0.674 \\
p=0.004\end{array}$ & $\begin{array}{l}r=-0.501 \\
p=0.048\end{array}$ & N.S.C & 31 & $\begin{array}{l}r=0.436 \\
p=0.05\end{array}$ & N.S.C. & N.S.C. \\
\hline
\end{tabular}

N.S.C = no significant correlation was observed.

*Correlation approaches significance.

In mature milk (W3), myristic (C14:0) is significantly and positively correlated with lutein and $\beta$-carotene concentrations. Arachidic acid (C20:0) was found to positively correlate significantly only with $\beta$-cryptoxanthin. Oleic acid (C18:1n-9), was the only MUFA to have an inverse correlation only with lutein and $\beta$-carotene. Zeaxanthin did not show a correlation with any individual fatty acids during week 3 . By contrast, in mature milk (W4), only zeaxanthin correlated positively and significantly with the lignoceric acid $(\mathrm{C} 24: 0)$. Zeaxanthin, $\beta$-cryptoxanthin, and $\beta$-carotene all 
positively correlate with eicosenoic acid (C20:1n-9) levels, although zeaxanthin did not reach a statistically significant level. Eicosadienoic acid (C20:2n-6) was the only PUFA that was revealed to be positively correlated with $\beta$-cryptoxanthin in mature milk (W4). Lutein was not found to be correlated with any individual fatty acid in mature milk during week 4 .

\subsection{Discussion and Conclusions}

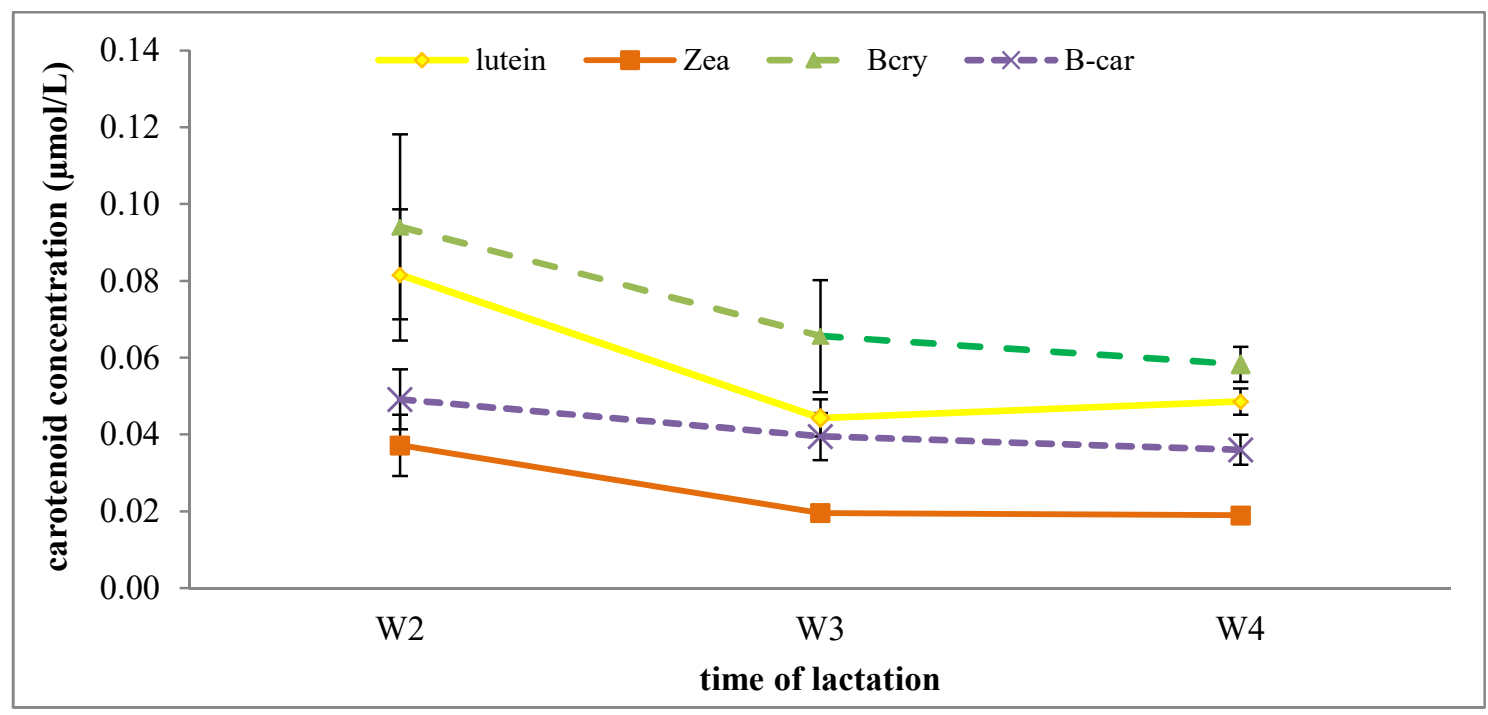

Figure 42. Carotenoid concentrations (mean $\pm \mathrm{SEM}$ ) in $\mu \mathrm{mol} / \mathrm{L}$ during the first month post-partum (data from Table 25).

A longitudinal decrease in carotenoid levels in human milk has been previously observed, with highest levels found in colostrum and the lowest in mature milk [135, 147]. We found that the most abundant carotenoids in the milk from the population of women in our study are lutein and $\beta$-cryptoxanthin. This apparent equivalence in the levels of the two carotenoids as seen in Figure 42 obscures an underlying and interesting difference. Although the average values of lutein and $\beta$-cryptoxanthin are nearly the 
same it is interesting to note that the $\beta$-cryptoxanthin levels are higher in $63 \%$ of all subjects in the study, see Table 33.

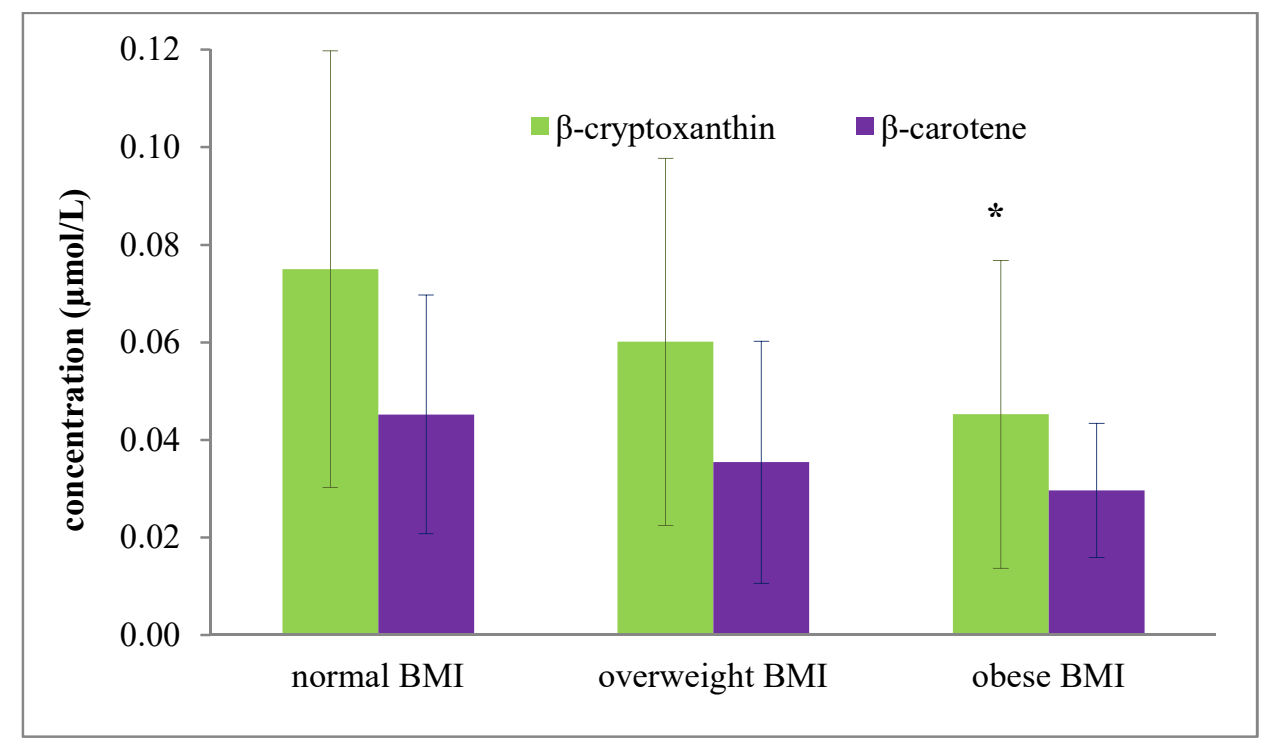

Figure 43. Comparison of pro-vitamin A carotenoids by BMI group (data from Table 26). Concentrations (mean \pm standard deviation).

* significantly different than normal group $(\mathrm{p}=0.03)$.

For data obtained for mature milk during week 4 we note that when grouped by BMI, see Table 26 , the concentration of $\beta$-cryptoxanthin was found to be highest $(0.08 \pm$ 0.04) in the normal BMI group, as compared to the overweight and obese groups, see Figure 43. These differences in carotenoid levels for those subjects with lower versus higher BMI's are consistent with the reports that linked serum carotenoid levels with BMI. $\beta$-Cryptoxanthin is converted to vitamin $\mathrm{A}$ in adipose tissue. For subjects having high BMI values, $\beta$-Cryptxanthin would be taken up from serum and metabolized, reducing amounts in these subjects available for transport into milk. We attribute the observation that lutein and $\beta$-cryptoxanthin are statistically similar in concentration over 
time to originate in this difference in the $\beta$-cryptoxanthin levels with BMI a trend that does not exist for lutein. If $\beta$-cryptoxanthin were not metabolized it would presumably be found in higher concentrations for all subjects.
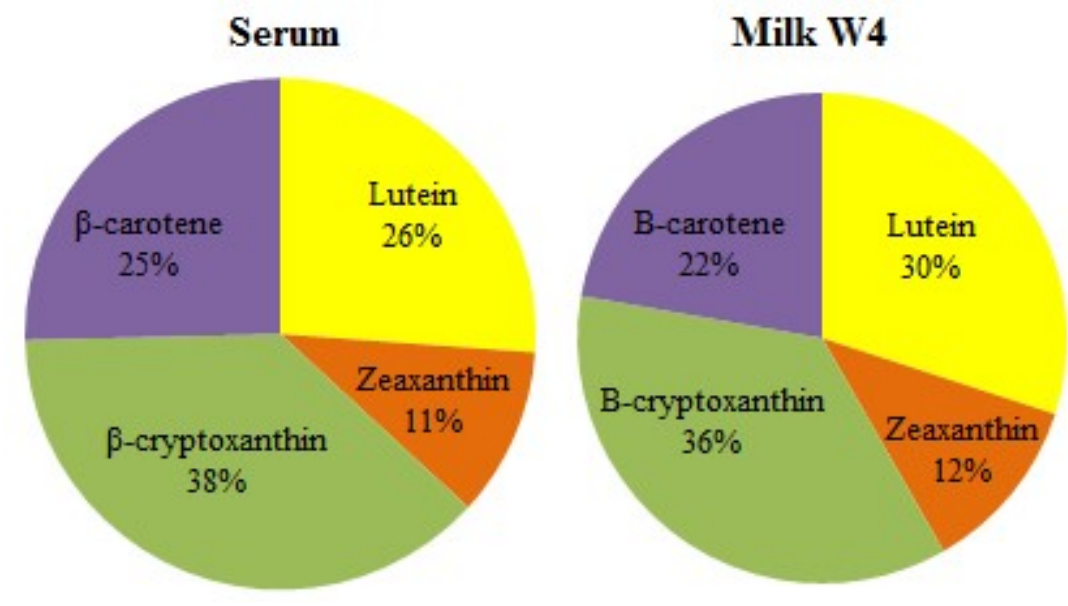

Figure 44. Composition of carotenoids in serum and mature milk during the 4 th week post-partum.

When comparing the percent composition of carotenoids in serum and milk, we observed that the concentration of lutein, relative to the other carotenoids, is higher in milk than in serum, see Figure 44. The proportion of lutein in mother's milk is higher than that in serum by a factor of 5.4, and the carotenoid concentration in milk is about 5-7 times less than in serum. Gossage et al.[152] studied the carotenoid composition in plasma of 16 lactating women and showed that the levels of lutein decreased between day $4^{\text {th }}$ and day $32^{\text {nd }}$ post-partum. That the lutein concentration decreases in serum while increasing in maternal milk, is evidence of an increased efficiency in maternal transfer of lutein from plasma to milk during the first month post-partum. In addition to our results, the study by Sherry et al.[153] reported that the levels of lutein and zeaxanthin were most 
abundant in breast milk (4-6 weeks post-partum) although the concentrations in milk tended to decrease over the study period ( 6 weeks). They did not see a change in maternal plasma during the period. These outcomes reveal that lutein is actively transported into milk during early stages of lactation (during the first month), after that the levels slowly decrease and reach a constant level. Even though the level of lutein decreases slowly in secreted milk, the active mobilization of the carotenoid appears to work against this trend as the mother's body-stores of carotenoids diminish providing the infant a the maximal amount of this nutritionally significant carotenoid.

Total fatty acids in human milk (principally present as triglycerides) do not decrease with time during lactation. This contrasts with the trend for carotenoids, shown previously in Chapter 3. Consequently, we do not find a correlation between the total $\%$ fatty acid and carotenoid levels. The overall transport of fatty acids from plasma into human milk does not account for a simple mechanism that controls transport of carotenoids during early lactation, despite the common lipophilic characteristics of both carotenoids and fatty acids. The mechanism of transport of carotenoids appears be regulated by factors other than those controlling total triglycerides in milk. Evidence from carotenoid levels during early lactation (in colostrum and to some extent transitional milk) indicates that carotenoid transport during this period may be controlled by different factors than in mature milk.

It is accepted that the source of carotenoids secreted into milk is largely the circulating maternal blood supply. To a large extent this is also true of many individual fatty acids including those considered essential. Although carotenoid concentrations do 
not correlate with total fat percentages in milk, strong correlations were found between carotenoid concentrations in maternal serum and milk (see Table 34). Essential fatty acids originate from dietary intake and to a lesser extent from mobilization from body stores. Their concentrations in milk are therefore, like carotenoids, dependent upon serum levels. In contrast to these essential fatty acids, many other fatty acids can be synthesized in situ by the mammary gland and/or by the liver in addition to originating from diet. Also depending on metabolic rates, their secreted concentrations in milk can be uncoupled from dietary intake and to an extent from serum concentrations.
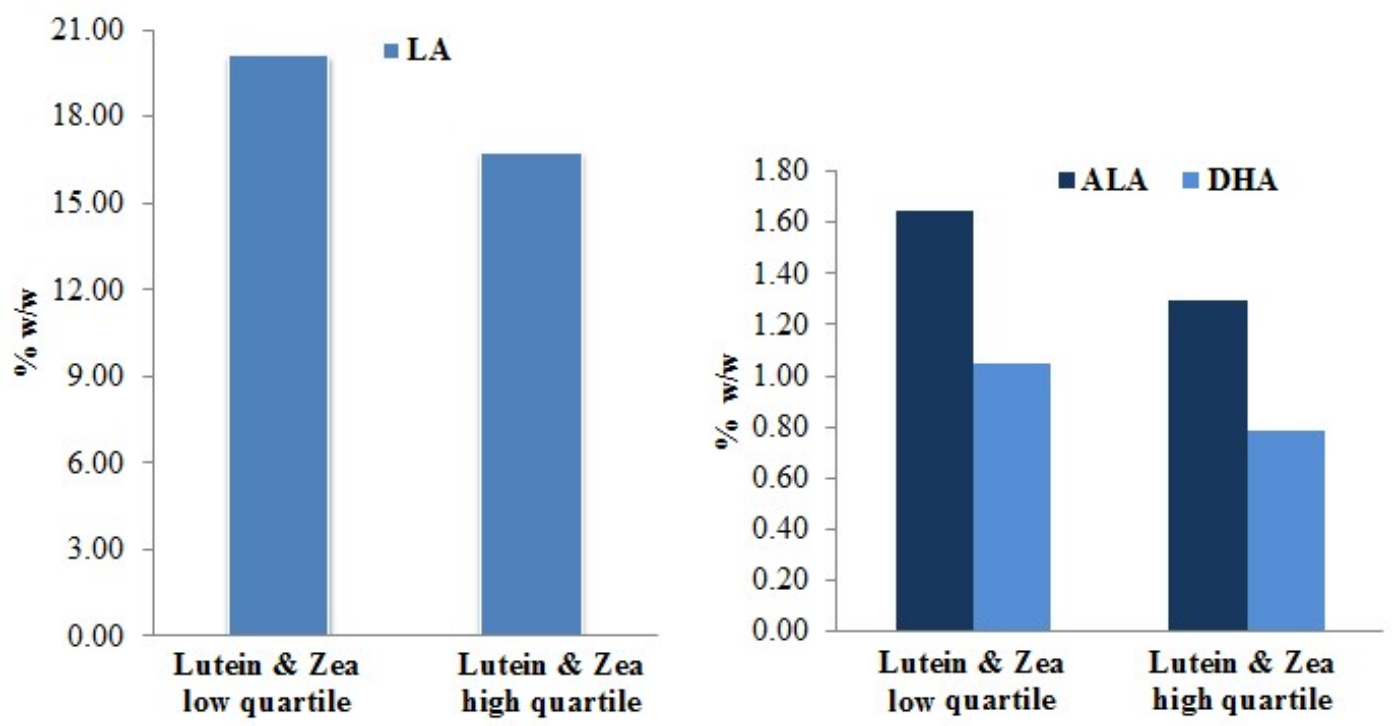

Figure 45. Relation of essential fatty acids with lutein and zeaxanthin in transitional milk.

We find that the concentrations of a small number of individual fatty acids do correlate, some positively, others negatively, with carotenoid concentrations in human milk, see Tables 7 and 8. Different classes of lipids differ in their physical and chemical properties the mechanisms of their transport into milk also differ at the molecular level. 
Our analysis shows the most statistically significant correlations between fatty acids and carotenoids are found in transitional milk (W2) as opposed to mature milk (W3 \& W4). In transitional milk, all four carotenoids measured in this study were positively correlated with myristic acid. Myristic acid originates from de novo synthesis in the mammary glands and liver. Only the non pro-vitamin A xanthophylls, lutein and zeaxanthin, were found to have a positive correlation with palmitic acid, which can likewise originate from de novo synthesis, but is also abundant in the diet. We observed an inverse correlation of lutein and zeaxanthin with the essential fatty acids, linoleic acid and $\alpha$-linoleic acid. As a consequence, higher concentrations of lutein and zeaxanthin are likely present when lower concentrations of these two essential fatty acids are present in maternal milk, see Figure 45. Lutein and zeaxanthin are known to be carried by high density lipoproteins in the circulating system. The inverse relationship between the carotenoids and the essential fatty acids suggests the possibility of a competition between these fats and the lutein and zeaxanthin for a critical transport step. HDL may have a limited transport capacity, and in the presence of high levels of essential PUFA, the capacity to transport carotenoids may be diminished. Another potential relationship that could link carotenoid levels and these fatty acids may be through the events associated with oxidative stress. N-6 fatty acids can potentiate oxidative processes and ROS production, leading to degradation of carotenoids. Further studies will be necessary to further investigate the possibility of a mechanistic association between these individual fatty acids and carotenoids. 


\section{SUMMARY}

We have reported the concentrations of 29 fatty acids in maternal milk. Special attention was given to the essential polyunsaturated fatty acids since they are known to play an important role in early development. In general, n-3 and n-6 polyunsaturated fatty acids were found to be abundant in maternal milk, which is a nutritional advantage for the new-born. The levels of these fatty acids are in accordance with the World Health Organization guidelines for intake by infants and values reported for other countries. Concentrations of individual fatty acids in this population, when compared with other previously studied groups, reveals a number of new trends. The DHA $(0.78 \% \mathrm{w} / \mathrm{w})$ concentration in the milk of these Peruvian women was found to fall into the highest quartile group among the 29 different countries studied.

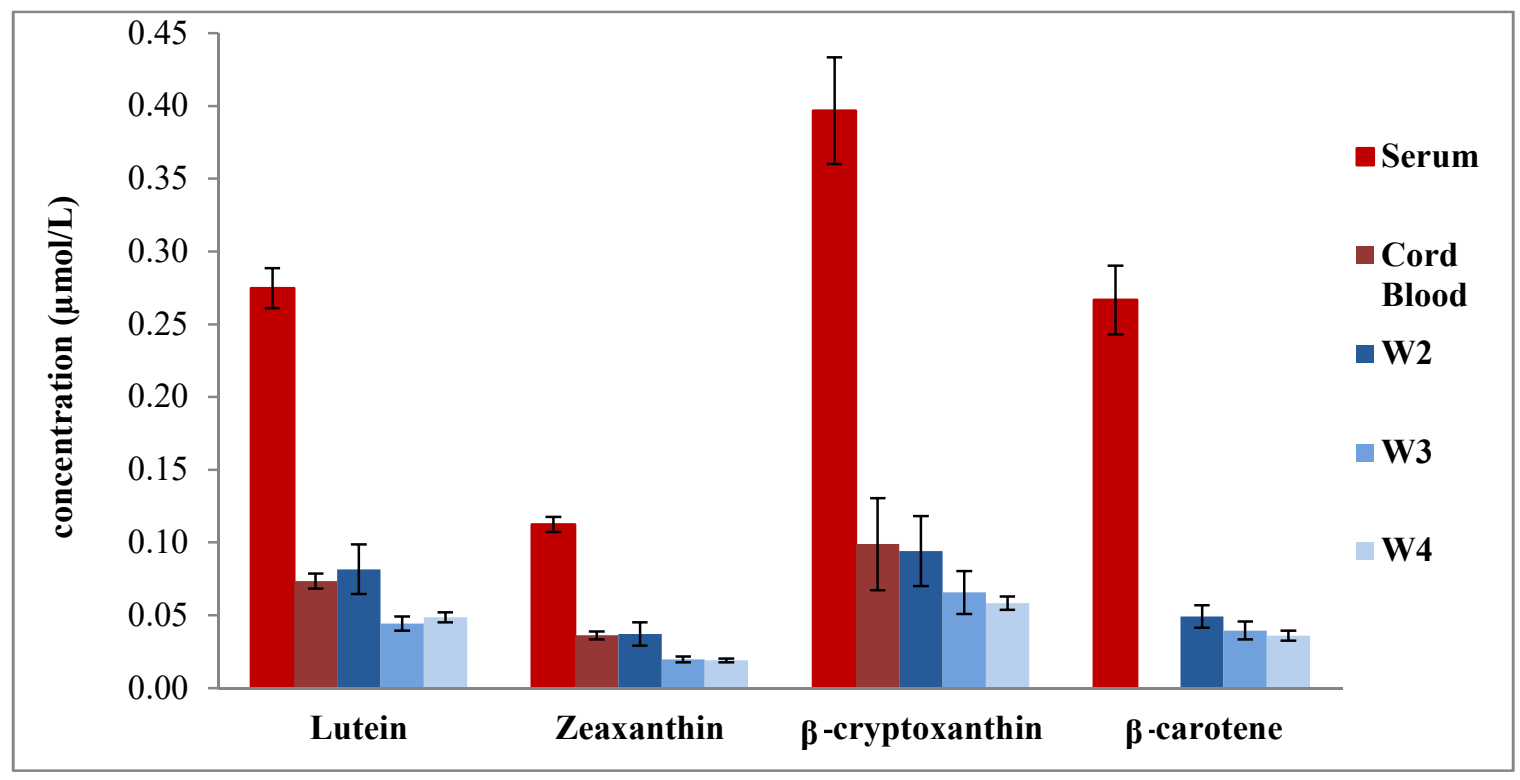

Figure 46. Carotenoid concentration in maternal milk, serum, and cord blood. Concentration (mean \pm S.E.M.). Serum, $n=74$; milk W2, $n=30$; milk W3, $n=28$; milk W4, $\mathrm{n}=74$; and cord blood, $\mathrm{n}=26$. 
Our measurements of the concentrations of carotenoids in maternal milk, maternal serum, and cord blood confirm that maternal serum concentrations are consistently higher than either cord blood or maternal milk concentrations. Although concentrations in maternal milk decrease with time post-partum, lutein:zeaxanthin ratios remain constant. Maternal serum carotenoid concentrations correlate with both cord blood and maternal milk concentrations.

We observed that the proportion of lutein in serum decreases as a fraction of total carotenoids during the first month, whereas in maternal milk the proportion of lutein increases during this period. Our results suggest that lutein is actively transported into maternal milk. Body mass index (BMI) influenced the concentration of pro-vitamin A carotenoids in milk and serum, but did not have a comparable effect on the concentration of the xanthophylls, lutein and zeaxanthin. For individuals with high BMI levels, provitamin A concentrations in maternal milk are lower than those with normal adiposity whereas lutein and zeaxanthin are identical in these two groups of women.

No correlation is found between $\%$ total fat in milk and carotenoid levels. The transfer of carotenoids from the maternal blood stream into milk is not controlled by a simple relationship with total fat in milk. We found a significant inverse correlation between lutein and the essential fatty acids, linoleic and $\alpha$-linoleic acid in transitional milk. A competition for transport between these the fatty acids and lutein is suggested by this relationship. Lutein and zeaxanthin concentrations in transitional milk $\left(2^{\text {nd }}\right.$ week post-partum) are found to be higher when linoleic and $\alpha$-linoleic acids are lower in concentration. The inverse relationship is observed in mature milk during the $4^{\text {th }}$ week of 
lactation. In mature milk the lutein concentration is lower and we observe that linoleic and $\alpha$-linoleic acids have high concentrations, see Figure 47. A paired t-test showed that lutein, zeaxanthin and $\alpha$-linoleic acid concentrations are statistically significantly different between transitional and mature milk $(\mathrm{p}<0.05)$. Linoleic acid showed had the same trend but it was not statistically significant $(\mathrm{p}=0.07)$.
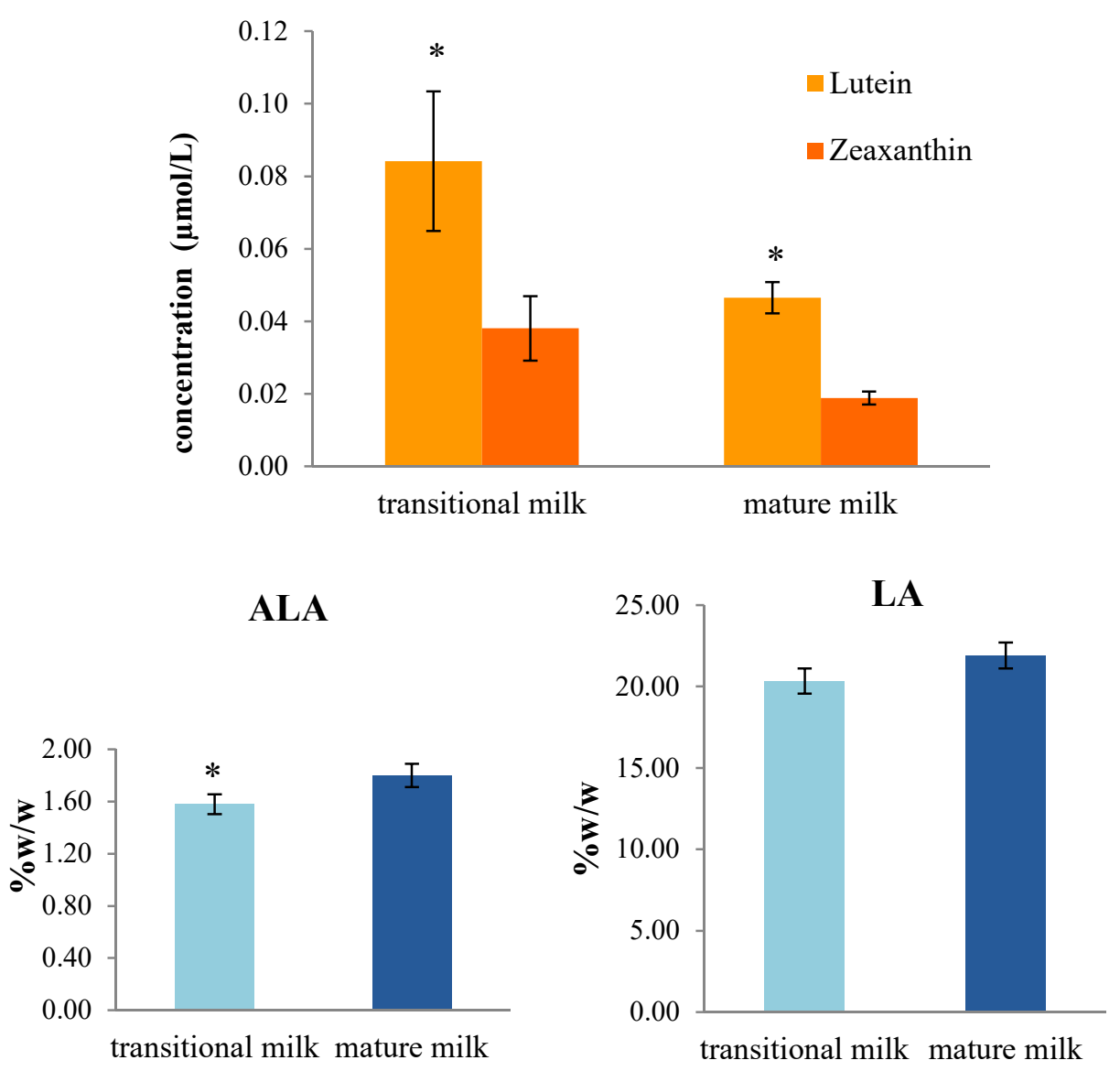

Figure 47. Lutein, zeaxanthin, $\alpha$-linoleic (ALA), and linoleic acid (LA) variations (mean \pm SEM) between transitional and mature milk.

*significantly different than mature milk $(\mathrm{p}<0.05)$. 
This is the first time a study has presented data comparing carotenoid concentrations to those of individual fatty acids in maternal milk. We conclude that carotenoid transport into milk during the early stages of lactation (colostrum and transitional milk) is influenced by different physiological factors than in mature milk. Although based on a limited sample, this study points to the need for further investigation into the association between individual fatty acids and carotenoid levels which may shed light on the relationship between transport of individual fatty acids and carotenoids. 


\section{REFERENCES}

1. Tapiero, H., D.M. Townsend, and K.D. Tew, The role of carotenoids in the prevention of human pathologies. Biomedicine \& Pharmacotherapy, 2004. 58(2): p. 100-110.

2. Perera, C.O. and G.M. Yen, Functional Properties of Carotenoids in Human Health. International Journal of Food Properties, 2007. 10(2): p. 201-230.

3. Haddad, N.F., et al., Lycopene and beta-carotene induce growth inhibition and proapoptotic effects on ACTH-secreting pituitary adenoma cells. PloS one, 2013. 8(5): p. e62773.

4. Khachik, F., G.R. Beecher, and J.C. Smith, Jr., Lutein, lycopene, and their oxidative metabolites in chemoprevention of cancer. J Cell Biochem Suppl, 1995. 22: p. 236-46.

5. Khachik, F., et al., Identification, quantification, and relative concentrations of carotenoids and their metabolites in human milk and serum. Anal Chem, 1997. 69(10): p. 1873-81.

6. Goodwin, T.W., The Biochemistry of the Carotenoids. Vol. 1. 1980, London: Chapman and Hall. pp. 377.

7. Cunningham Jr, F. and E. Gantt, Genes and enzymes of carotenoid biosynthesis in plants. Annual review of plant biology, 1998. 49(1): p. 557-583.

8. Hirschberg, J., Carotenoid biosynthesis in flowering plants. Current opinion in plant biology, 2001. 4(3): p. 210-218.

9. Sandmann, G., Carotenoid biosynthesis in microorganisms and plants, in EJB Reviews 1994. 1994, Springer. p. 129-146.

10. Delgado-Vargas, F., A. Jiménez, and O. Paredes-López, Natural pigments: carotenoids, anthocyanins, and betalains-characteristics, biosynthesis, processing, and stability. Critical reviews in food science and nutrition, 2000. 40(3): p. 173-289.

11. Britton, G., Structure and properties of carotenoids in relation to function. The FASEB Journal, 1995. 9(15): p. 1551-1558.

12. Subczynski, W.K., et al., Effects of polar carotenoids on dimyristoylphosphatidylcholine membranes: a spin-label study. Biochim Biophys Acta, 1992. 1105(1): p. 97-108. 
13. Furr, H.C., Analysis of retinoids and carotenoids: problems resolved and unsolved. The Journal of Nutrition, 2004. 134(1): p. 281S-285S.

14. Goodwin, T., Nature and properties, in The Biochemistry of the Carotenoids. 1980, Springer. p. 1-32.

15. Krinsky, N.I., Singlet oxygen in biological systems. Trends in Biochemical Sciences, 1977. 2(2): p. 35-38.

16. Krinsky, N.I., Carotenoids as chemopreventive agents. Prev Med, 1989. 18(5): p. 592-602.

17. Foote, C.S., Y.C. Chang, and R.W. Denny, Chemistry of Singlet Oxygen. X. Carotenoid Quenching Parallels Biological Protection. J. Am. Chem. Soc., 1970. 92: p. 5216-5218.

18. Stahl, W. and H. Sies, Antioxidant activity of carotenoids. Molecular aspects of medicine, 2003. 24(6): p. 345-351.

19. Sies, H. and W. Stahl, Vitamins $E$ and $C$, beta-carotene, and other carotenoids as antioxidants. The American journal of clinical nutrition, 1995. 62(6): p. 1315S$1321 \mathrm{~S}$.

20. Bagchi, K. and S. Puri, Free radicals and antioxidants in health and disease. Eastern Mediterranean health journal, 1998. 4(2): p. 350-360.

21. Mayne, S.T., Beta-carotene, carotenoids, and disease prevention in humans. The FASEB Journal, 1996. 10(7): p. 690-701.

22. Rao, A.V. and L.G. Rao, Carotenoids and human health. Pharmacological research, 2007. 55(3): p. 207-216.

23. Yamaguchi, M., Role of carotenoid $\beta$-cryptoxanthin in bone homeostasis. Journal of biomedical science, 2012. 19(1): p. 1.

24. Lee, C.M., et al., Review of animal models in carotenoid research. The Journal of nutrition, 1999. 129(12): p. 2271-2277.

25. Kotake-Nara, E. and A. Nagao, Absorption and metabolism of xanthophylls. Marine drugs, 2011. 9(6): p. 1024-1037.

26. Yonekura, L. and A. Nagao, Intestinal absorption of dietary carotenoids. Molecular nutrition \& food research, 2007. 51(1): p. 107-115. 
27. Yeum, K.-J. and R.M. Russell, Carotenoid bioavailability and bioconversion. Annual Review of Nutrition, 2002. 22(1): p. 483-504.

28. Sugawara, T., et al., Lysophosphatidylcholine enhances carotenoid uptake from mixed micelles by CaCo-2 human intestinal cells. J. Nutr., 2001. 131: p. 2921-27.

29. Harrison, E.H., Mechanisms involved in the intestinal absorption of dietary vitamin A and provitamin A carotenoids. Biochimica et Biophysica Acta (BBA)Molecular and Cell Biology of Lipids, 2012. 1821(1): p. 70-77.

30. CAROTENOIDS, I.P.A., Bioconversion of provitamin A carotenoids. Carotenoids in health and disease, 2004: p. 295.

31. US Department of Agriculture, A.R.S. USDA Nutrient Database for Standard Reference Release, 17, 2004; Available from: http://www.nal.usda.gov/fnic/foofcomp.

32. Bone, R.A., et al., Stereochemistry of the human macular carotenoids. Investigative Ophthalmology \& Visual Science, 1993. 34(6): p. 2033-40.

33. Schalch, W., R. Bone, and J. Landrum, The functional role of xanthophylls in the primate retina. Carotenoids: Physical, Chemical and Biological Functions and Properties, 2010: p. 257-282.

34. Landrum, J.T., et al., Carotenoids in the human retina, in Pure and Applied Chemistry. 1999. p. 2237.

35. Li, B., P. Vachali, and P.S. Bernstein, Human ocular carotenoid-binding proteins. Photochemical \& Photobiological Sciences, 2010. 9(11): p. 1418-1425.

36. Landrum, J.T., et al., Analysis of Zeaxanthin Distribution within Individual Human Retinas, in Methods in Enzymology, L. Packer, Editor. 1999, Academic Press: San Diego. p. 457-467.

37. Bone, R.A., et al., Analysis of the Macular Pigment by HPLC: Retinal Distribution and Age Study. Invest. Ophthalmol. Vis. Sci., 1988. 29: p. 843-849.

38. Provis, J.M., C.M. Diaz, and B. Dreher, Ontogeny of the primate fovea: a central issue in retinal development. Progress in Neurobiology, 1998. 54(5): p. 549-581.

39. Leung, I.Y.-F., et al., Nutritional manipulation of primate retinas, II: Effects of age, $n-3$ fatty acids, lutein, and zeaxanthin on retinal pigment epithelium. Investigative Ophthalmology \& Visual Science, 2004. 45(9): p. 3244-3256. 
40. Hammond, B.R., Possible role for dietary lutein and zeaxanthin in visual development. Nutrition reviews, 2008. 66(12): p. 695-702.

41. Vishwanathan, R., et al., Lutein and Preterm Infants With Decreased Concentrations of Brain Carotenoids. Journal of Pediatric Gastroenterology and Nutrition, 2014. 59(5): p. 659-665.

42. Lieblein-Boff, J.C., et al., Exploratory metabolomic analyses reveal compounds correlated with lutein concentration in frontal cortex, hippocampus, and occipital cortex of human infant brain. PloS one, 2015. 10(8): p. e0136904.

43. Vishwanathan, R., et al., Macular lutein and zeaxanthin are related to brain lutein and zeaxanthin in primates. Nutritional neuroscience, 2013. 16(1): p. 2129.

44. Johnson, E.J., Role of lutein and zeaxanthin in visual and cognitive function throughout the lifespan. Nutrition reviews, 2014. 72(9): p. 605-612.

45. Li, S.-Y., et al., Effect of lutein on retinal neurons and oxidative stress in a model of acute retinal ischemia/reperfusion. Investigative Ophthalmology \& Visual Science, 2009. 50(2): p. 836-843.

46. Li, S.-Y., et al., Lutein enhances survival and reduces neuronal damage in a mouse model of ischemic stroke. Neurobiology of disease, 2012. 45(1): p. 624632.

47. Parker, R.S., Carotenoids in Human Blood and Tissues. Am. Instutute of Nutr., 1989. NA: p. 101-104.

48. Ferruzzi, M.G. and S.J. Schwartz, Methodology for Assessment of Carotenoid Levels in Blood Plasma and Plasma Fractions. Carotenoids in health and disease, 2004: p. 85.

49. Maiani, G., et al., Carotenoids: actual knowledge on food sources, intakes, stability and bioavailability and their protective role in humans. Molecular nutrition \& food research, 2009. 53(S2): p. S194-S218.

50. Dachtler, M., et al., Combined HPLC-MS and HPLC-NMR on-line coupling for the separation and determination of lutein and zeaxanthin stereoisomers in spinach and in retina. Analytical chemistry, 2001. 73(3): p. 667-674.

51. Zhang, C., et al., Plasma concentrations of carotenoids, retinol, and tocopherols in preeclamptic and normotensive pregnant women. American journal of epidemiology, 2001. 153(6): p. 572-580. 
52. Ziari, S.A., et al., Serum vitamin A, vitamin E, and beta-carotene levels in preeclamptic women in northern Nigeria. American journal of perinatology, 1996. 13(05): p. 287-291.

53. Mikhail, M.S., et al., Preeclampsia and antioxidant nutrients: Decreased plasma levels of reduced ascorbic acid, (-tocopherol, and beta-carotene in women with preeclampsia. Am. J. Obstet. Gynecol., 1994. NA: p. 150-157.

54. Palan, P.R., M.S. Mikhail, and S.L. Romney, Placental and serum levels of carotenoids in preeclampsia. Obstetrics \& Gynecology, 2001. 98(3): p. 459-462.

55. Behrman, R.E. and A.S. Butler, Mortality and acute complications in preterm infants. 2007.

56. Al-Gubory, K.H., P.A. Fowler, and C. Garrel, The roles of cellular reactive oxygen species, oxidative stress and antioxidants in pregnancy outcomes. The international journal of biochemistry \& cell biology, 2010. 42(10): p. 1634-1650.

57. Yeum, K.J., et al., Relationship of plasma carotenoids, retinol and tocopherols in mothers and newborn infants. J Am Coll Nutr, 1998. 17(5): p. 442-7.

58. Oostenbrug, G.S., et al., Maternal and neonatal plasma antioxidant levels in normal pregnancy, and the relationship with fatty acid unsaturation. British Journal of Nutrition, 1998. 80(01): p. 67-73.

59. Oostenbrug, G., et al., Pregnancy-induced hypertension: maternal and neonatal plasma lipid-soluble antioxidant levels and its relationship with fatty acid unsaturation. European journal of clinical nutrition, 1998. 52(10): p. 754-759.

60. Kiely, M., et al., Concentrations of tocopherols and carotenoids in maternal and cord blood plasma. Eur J Clin Nutr, 1999. 53(9): p. 711-5.

61. Ibrahim, K., T.J. Hassan, and S.N. Jafarey, Plasma vitamin A and carotene in maternal and cord blood. Asia-Oceania Journal of Obstetrics and Gynaecology, 1991. 17(2): p. 159-164.

62. Bolisetty, S., et al., Postnatal changes in maternal and neonatal plasma antioxidant vitamins and the influence of smoking. Archives of Disease in Childhood-Fetal and Neonatal Edition, 2002. 86(1): p. F36-F40.

63. Herrera, E., et al., Relationship between plasma fatty acid profile and antioxidant vitamins during normal pregnancy. European journal of clinical nutrition, 2004. 58(9): p. 1231-1238. 
64. Dimenstein, R., et al., Effect of subadequate maternal vitamin-A status on placental transfer of retinol and beta-carotene to the human fetus. Neonatology, 1996. 69(4): p. 230-234.

65. Lindeman, J., et al., The total free radical trapping ability of cord blood plasma in preterm and term babies. Pediatric research, 1989. 26(1): p. 20-24.

66. Butte, N. and D.H. Calloway, Proteins, vitamin A, carotene, folacin, ferritin and zinc in Navajo maternal and cord blood. Neonatology, 1982. 41(5-6): p. 273-278.

67. Breithaupt, D.E. and A. Bamedi, Carotenoid esters in vegetables and fruits: $a$ screening with emphasis on $\beta$-cryptoxanthin esters. Journal of Agricultural and Food Chemistry, 2001. 49(4): p. 2064-2070.

68. Bone, R.A., et al., Lutein and Zeaxanthin in Eyes, Serum and Diet of Human Subjects. Exp. Eye Res., 2000. 71: p. 239-245.

69. Schweigert, F., et al., Effect of the stage of lactation in humans on carotenoid levels in milk, blood plasma and plasma lipoprotein fractions. European Journal of Nutrition, 2004. 43(1): p. 39-44.

70. Ribaya-Mercado, J.D., et al., Relationships of body mass index with serum carotenoids, tocopherols and retinol at steady-state and in response to a carotenoid-rich vegetable diet intervention in Filipino schoolchildren1. Bioscience reports, 2008. 28(2): p. 97-106.

71. Andersen, L.F., et al., Longitudinal associations between body mass index and serum carotenoids: the CARDIA study. British Journal of Nutrition, 2006. 95(02): p. 358-365.

72. Brady, W.E., et al., Human serum carotenoid concentrations are related to physiologic and lifestyle factors. The Journal of Nutrition, 1996. 126(1): p. 129.

73. Grune, T., et al., $\beta$-Carotene is an important vitamin A source for humans. The Journal of Nutrition, 2010. 140(12): p. 2268S-2285S.

74. Picone, S., et al., Arterial cord blood lutein levels in preterm and term healthy newborns are sex and gestational age dependent. Clinical biochemistry, 2012. 45(18): p. 1558-1563.

75. Jensen, R.G., Lipids in human milk. Lipids, 1999. 34(12): p. 1243-1271.

76. Schanler, R.J., The Use of Human Milk for Premature Infants. Pediatr. Clin. N. Am., 2001. 48(1): p. 207-219. 
77. Jensen, R.G., The Lipids of Human Milk. 1989: CRC Press.

78. Nelson, D.L., A.L. Lehninger, and M.M. Cox, Lehninger principles of biochemistry. 2008: Macmillan.

79. Rustan, A.C. and C.A. Drevon, Fatty Acids: Structures and Properties, in eLS. 2001, John Wiley \& Sons, Ltd.

80. White, B., Dietary fatty acids. American family physician, 2009. 80(4).

81. Mensink, R.P. and M.B. Katan, Effect of dietary fatty acids on serum lipids and lipoproteins. A meta-analysis of 27 trials. Arteriosclerosis, Thrombosis, and Vascular Biology, 1992. 12(8): p. 911-919.

82. Hu, F.B., et al., Dietary fat intake and the risk of coronary heart disease in women. New England Journal of Medicine, 1997. 337(21): p. 1491-1499.

83. Legrand, P. and V. Rioux, Specific roles of saturated fatty acids: Beyond epidemiological data. European Journal of Lipid Science and Technology, 2015. 117(10): p. 1489-1499.

84. Kris-Etherton, P.M. and N. Committee, Monounsaturated fatty acids and risk of cardiovascular disease. Circulation, 1999. 100(11): p. 1253-1258.

85. Roche, H.M., Unsaturated fatty acids. Proceedings of the Nutrition Society, 1999. 58(02): p. 397-401.

86. James, M.I.G.a.A.T., Lipid Biochemistry: An Introduction. 3rd. ed. 1980, London: Chapman and Hall. 247.

87. Lopez, C. and O. Ménard, Human milk fat globules: Polar lipid composition and in situ structural investigations revealing the heterogeneous distribution of proteins and the lateral segregation of sphingomyelin in the biological membrane. Colloids and Surfaces B: Biointerfaces, 2011. 83(1): p. 29-41.

88. Lopez, C., Milk fat globules enveloped by their biological membrane: Unique colloidal assemblies with a specific composition and structure. Current Opinion in Colloid \& Interface Science, 2011. 16(5): p. 391-404.

89. Koletzko, B., et al., Physiological aspects of human milk lipids. Early Human Development, 2001. 65, Supplement 2: p. S3-S18.

90. Kim, J. and J. Friel, Lipids and Human Milk. Lipid Technology, 2012. 24(5): p. 103-105. 
91. Bauman, D.E. and J.M. Griinari, NUTRITIONAL REGULATION OF MILK FAT SYNTHESIS. Annual Review of Nutrition, 2003. 23(1): p. 203-227.

92. Bitman, J., et al., Comparison of the lipid composition of breast milk from mothers of term and preterm infants. The American Journal of Clinical Nutrition, 1983. 38(2): p. 300-12.

93. Martin, J.-C., et al., Triacylglycerol structure of human colostrum and mature milk. Lipids, 1993. 28(7): p. 637-643.

94. Lopez-Lopez, A., et al., Fatty acid and sn-2 fatty acid composition in human milk from Granada (Spain) and in infant formulas. European journal of clinical nutrition, 2002. 56: p. 1242-1254.

95. Haddad, I., M. Mozzon, and N.G. Frega, Trends in fatty acids positional distribution in human colostrum, transitional, and mature milk. European Food Research and Technology, 2012. 235(2): p. 325-332.

96. Innis, S.M., Human milk: maternal dietary lipids and infant development. Proceedings of the Nutrition Society, 2007. 66(03): p. 397-404.

97. Koletzko, B., et al., The roles of long-chain polyunsaturated fatty acids in pregnancy, lactation and infancy. Journal of Perinatal Medicine, 2008. 36(1): p. 5-14.

98. E. Patterson, R.W., G. F. Fitzgerald, R. P. Ross, and C. Stanton, Health Implications of High Dietary Omega-6 Polyunsaturated Fatty Acids. Journal of Nutrition and Metabolism, 2012. 2012: p. 16.

99. Innis, S.M., Dietary (n-3) fatty acids and brain development. The Journal of Nutrition, 2007. 137(4): p. 855-859.

100. Niu, S.-L., et al., Reduced G protein-coupled signaling efficiency in retinal rod outer segments in response to $n-3$ fatty acid deficiency. Journal of Biological Chemistry, 2004. 279(30): p. 31098-31104.

101. Precht, D. and J. Molkentin, Trans fatty acids in human milk. European Journal of Lipid Science and Technology, 2000. 102(10): p. 635-640.

102. Craig-Schmidt, M.C., World-wide consumption of trans fatty acids. Atherosclerosis Supplements, 2006. 7(2): p. 1-4.

103. Jensen, R.G., M.A. McGuire, and M.K. McGuire, Trans fatty acids in human milk. European Journal of Lipid Science and Technology, 2000. 102(10): p. 640642. 
104. Yuhas, R., K. Pramuk, and E.L. Lien, Human milk fatty acid composition from nine countries varies most in DHA. Lipids, 2006. 41(9): p. 851-858.

105. Glew, R.H., et al., Trans fatty acids and conjugated linoleic acids in the milk of urban women and nomadic Fulani of northern Nigeria. Clinica chimica acta, 2006. 367(1): p. 48-54.

106. Bahrami, G. and Z. Rahimi, Fatty acid composition of human milk in Western Iran. European journal of clinical nutrition, 2005. 59(4): p. 494-497.

107. Xiang, M., et al., Long-chain polyunsaturated fatty acids in human milk and brain growth during early infancy. Acta Paediatrica, 2000. 89(2): p. 142-147.

108. Marin, M.C., et al., [Fatty acid composition of human milk from mothers of preterm and full-term infants]. Archivos argentinos de pediatria, 2009. 107(4): p. 315-320.

109. Makrides, M., et al., Changes in the polyunsaturated fatty acids of breast milk from mothers of full-term infants over $30 \mathrm{wk}$ of lactation. The American Journal of Clinical Nutrition, 1995. 61(6): p. 1231-1233.

110. Wan, Z.-X., et al., Lipid content and fatty acids composition of mature human milk in rural North China. British Journal of Nutrition, 2010. 103(06): p. 913916.

111. Roy, S., P. Dhar, and S. Ghosh, Comparative evaluation of essential fatty acid composition of mothers' milk of some urban and suburban regions of West Bengal, India. International journal of food sciences and nutrition, 2012. 63(8): p. 895-901.

112. Al-Tamer, Y. and A. Mahmood, The influence of Iraqi mothers' socioeconomic status on their milk-lipid content. European journal of clinical nutrition, 2006. 60(12): p. 1400-1405.

113. Genzel-Boroviczényy, O., J. Wahle, and B. Koletzko, Fatty acid composition of human milk during the 1st month after term and preterm delivery. European journal of pediatrics, 1997. 156(2): p. 142-147.

114. Martin, M.A., et al., Fatty acid composition in the mature milk of Bolivian forager-horticulturalists: controlled comparisons with a US sample. Maternal \& child nutrition, 2012. 8(3): p. 404-418.

115. Pugo-Gunsam, P., et al., Fatty acid composition of white adipose tissue and breast milk of Mauritian and French mothers and erythrocyte phospholipids of 
their full-term breast-fed infants. British Journal of Nutrition, 1999. 82(04): p. 263-271.

116. Schmeits, B.L., et al., Fatty acid composition of the milk lipids of women in Nepal. Nutrition research, 1999. 19(9): p. 1339-1348.

117. Saphier, O., et al., Fatty acid composition of breastmilk of Israeli mothers. Indian pediatrics, 2013. 50(11): p. 1044-1046.

118. Nishimura, R.Y., et al., Breast milk fatty acid composition of women living far from the coastal area in Brazil. Jornal de pediatria, 2013. 89(3): p. 263-268.

119. Aydin, I., et al., Comparing the fatty acid levels of preterm and term breast milk in Turkish women. Turkish journal of medical sciences, 2014. 44(2): p. 305-310.

120. Ribeiro, M., et al., Fatty acid profile of human milk of Portuguese lactating women: prospective study from the 1st to the 16th week of lactation. Annals of Nutrition and Metabolism, 2008. 53(1): p. 50-56.

121. Serra, G., et al., Fatty acid composition of human milk in Italy. Neonatology, 1997. 72(1): p. 1-8.

122. Krešić, G., et al., Dietary and breast milk trans fatty acids seen in Croatian breastfeeding women from Adriatic region. Journal of Food \& Nutrition Research, 2013. 52(3).

123. Aleksandra, A., et al., Milk in human nutrition: comparison of fatty acid profiles. Acta Veterinaria, 2009. 59.

124. Decsi T, O.S., Molnár S, Burus I., Fatty acid composition of human milk in Hungary. Acta Paediatrica, 2000. 89(11): p. 1394-5.

125. Boersma, E.R., et al., Vitamin E, lipid fractions, and fatty acid composition of colostrum, transitional milk, and mature milk: an international comparative study. The American Journal of Clinical Nutrition, 1991. 53(5): p. 1197-1204.

126. Brenna, J.T., et al., Docosahexaenoic and arachidonic acid concentrations in human breast milk worldwide. The American Journal of Clinical Nutrition, 2007. 85(6): p. 1457-1464.

127. Del Prado, M., et al., Contribution of dietary and newly formed arachidonic acid to human milk lipids in women eating a low-fat diet. The American Journal of Clinical Nutrition, 2001. 74(2): p. 242-247. 
128. Makrides, M., M. Neumann, and R.A. Gibson, Effect of maternal docosahexaenoic acid (DHA) supplementation on breast milk composition. European journal of clinical nutrition, 1996. 50(6): p. 352-357.

129. Carlson, S.E., Docosahexaenoic acid supplementation in pregnancy and lactation. The American Journal of Clinical Nutrition, 2009. 89(2): p. 678S-684S.

130. López-López, A., A.I. Castellote-Bargalló, and M.C. López-Sabater, Comparison of two direct methods for the determination of fatty acids in human milk. Chromatographia, 2001. 54(11-12): p. 743-747.

131. Cantellops, D., et al., Determination of lipids in infant formula powder by direct extraction methylation of lipids and fatty acid methyl esters (FAME) analysis by gas chromatography. JOURNAL-AOAC INTERNATIONAL, 1999. 82: p. 11281139.

132. Huber, L., Validation of Analytical Methods. 2010, Agilent Technologies: Germany.

133. HARMONISATION, I.C.O., VALIDATION OF ANALYTICAL PROCEDURES: TEXT AND METHODOLOGY Q2(RI) I.H.T. GUIDELINE, Editor. 2005, INTERNATIONAL CONFERENCE ON HARMONISATION OF TECHNICAL REQUIREMENTS FOR REGISTRATION OF PHARMACEUTICALS FOR HUMAN USE: Geneva, Switzerland. p. 18.

134. Berenhauser, A.C., et al., Fatty acid composition in preterm and term breast milk. International journal of food sciences and nutrition, 2012. 63(3): p. 318-325.

135. Macias, C. and F.J. Schweigert, Changes in the concentration of carotenoids, vitamin A, alpha-tocopherol and total lipids in human milk throughout early lactation. Annals of nutrition and metabolism, 2001. 45(2): p. 82-85.

136. Samur, G., A. Topcu, and S. Turan, Trans fatty acids and fatty acid composition of mature breast milk in turkish women and their association with maternal diet's. Lipids, 2009. 44(5): p. 405-413.

137. Mueller, A., et al., Trans Fatty Acids in Human Milk are an Indicator of Different Maternal Dietary Sources Containing Trans Fatty Acids. Lipids, 2010. 45(3): p. 245-251.

138. Doell, D., et al., Updated estimate of trans fat intake by the US population. Food Additives \& Contaminants: Part A, 2012. 29(6): p. 861-874.

139. INEI, Peru: consumo percapita de los principales alimentos 2008-2009. 2010, Instituto Nacional de Estadistica e Informatica: Lima. p. 32. 
140. Trumbo, P., et al., Food and Nutrition Board of the Institute of Medicine, The National Academies. Dietary reference intakes for energy, carbohydrate, fiber, fat, fatty acids, cholesterol, protein and amino acids. J Am Diet Assoc, 2002. 102(11): p. 1621-30.

141. 2016/127, C.D.R.E., Regulations: supplementing Regulation (EU) No 609/2013 of the European Parliament and of the Council as regards the specific compositional and information requirements for infant formula and follow-on formula and as regards requirements on information relating to infant and young child feeding, in L 25, E. Union, Editor. 2016, Publications Office of the European Union Luxembourg.

142. omega-3, G.O.f.E.a.D. Global Recommendations for EPA and DHA Intake Rev 18 March 2015; Available from: http://www.iffo.net/system/files/A\%206\%20Summary\%20GOED\%20For\%20IA DSA \%20only\%20english.pdf.

143. Emmett, P.M. and I.S. Rogers, Properties of human milk and their relationship with maternal nutrition. Early Hum. Dev., 1997. 49 p. s7-s28.

144. Picciano, M.F., Pregnancy and lactation: physiological adjustments, nutritional requirements and the role of dietary supplements. The Journal of Nutrition, 2003. 133(6): p. 1997S-2002S.

145. Patton, S., et al., Carotenoids in Human Colostrum. Lipids, 1990. 25(3): p. 159165.

146. Gossage, C.P., et al., Carotenoid composition of human milk during the first month postpartum and the response to beta-carotene supplementation. Am J Clin Nutr, 2002. 76(1): p. 193-7.

147. Schweigert, F.J., et al., Effect of the stage of lactation in humans on carotenoid levels in milk, blood plasma and plasma lipoprotein fractions. Eur J Nutr, 2004. 43(1): p. 39-44.

148. Canfield, L.M., et al., Multinational study of major breast milk carotenoids of healthy mothers. Eur J Nutr, 2003. 42(3): p. 133-41.

149. de Azeredo, V.B. and N.M. Trugo, Retinol, carotenoids, and tocopherols in the milk of lactating adolescents and relationships with plasma concentrations. Nutrition, 2008. 24(2): p. 133-139.

150. Meneses, F. and N.M.F. Trugo, Retinol, b-carotene, and lutein + zeaxanthin in the milk of Brazilian nursing women: associations with plasma concentrations 
and influences of maternal characteristics. Nutrition Research, 2005. 25: p. 443451.

151. Jackson, J., et al., Major Carotenoids in Mature Human Milk: Longitudinal and Diurnal Patterns. Nutritional Biochemistry, 1998. 9: p. 2-7.

152. Gossage, C., et al., Effect of $\beta$-carotene supplementation and lactation on carotenoid metabolism and mitogenic T lymphocyte proliferation. The American journal of clinical nutrition, 2000. 71(4): p. 950-955.

153. Sherry, C.L., et al., Lutein supplementation increases breast milk and plasma lutein concentrations in lactating women and infant plasma concentrations but does not affect other carotenoids. The Journal of Nutrition, 2014. 144(8): p. 12561263. 


\section{APPENDICES}

\section{Fatty acids in human milk found in countries in South and North America.}

\begin{tabular}{|l|ccc|}
\hline $\begin{array}{l}\text { North } \\
\text { America }\end{array}$ & Canada & \multicolumn{1}{c}{ US } & Mexico \\
\hline Author & Yuhas & Yuhas & Yuhas \\
\hline lactation & & & \\
stage & mature & mature & mature \\
N & \multicolumn{1}{c}{48} & \multicolumn{1}{c}{49} & \multicolumn{1}{c|}{46} \\
\hline C12:0 & 5.25 & 4.40 & 4.97 \\
C14:0 & 5.84 & 4.91 & 5.57 \\
C16:0 & 18.67 & 19.26 & 19.91 \\
C18:0 & 5.83 & 6.21 & 6.07 \\
total SFA & 38.47 & 37.41 & 39.16 \\
C16:1n-7 & 2.79 & 2.64 & 2.64 \\
C16:1n-9 & 0.23 & 0.44 & 0.35 \\
C18:1n-9 & 35.18 & 32.77 & 30.79 \\
C20:n-9 & 0.52 & 0.39 & 0.42 \\
total & & & \\
MUFA & 42.89 & 40.40 & 38.11 \\
C18:2n-6 & & & \\
LA & 11.48 & 14.78 & 16.05 \\
C20:3n-6 & 0.27 & 0.35 & 0.33 \\
C20:4n-6 & & & \\
AA & 0.37 & 0.45 & 0.42 \\
total n-6 & 13.42 & 17.19 & 18.26 \\
C18:3n-3 & & & \\
ALA & 1.22 & 1.05 & 1.05 \\
C20:5n-3 & & & \\
EPA & 0.08 & 0.07 & 0.07 \\
C22:6n-3 & & & \\
DHA & 0.17 & 0.17 & 0.26 \\
total n-3 & 1.77 & 1.56 & 1.69 \\
\hline
\end{tabular}

\begin{tabular}{|c|c|c|c|c|}
\hline $\begin{array}{l}\text { South } \\
\text { America } \\
\end{array}$ & Chile & Argentina & Bolivia & Brazil \\
\hline Author & Yuhas & Marin & Martin & Nishimura \\
\hline $\begin{array}{l}\text { lactation } \\
\text { stage }\end{array}$ & mature & $\begin{array}{c}\text { 15day- } \\
\text { 3months }\end{array}$ & & \\
\hline $\mathbf{N}$ & 50 & 35 & 35 & 47 \\
\hline C12:0 & 6.15 & 5.10 & 5.72 & 7.46 \\
\hline C14:0 & 6.80 & 6.07 & 9.81 & 6.81 \\
\hline C16:0 & 18.79 & 21.23 & 24.96 & 19.50 \\
\hline C18:0 & 5.77 & 10.55 & 5.54 & 5.82 \\
\hline total SFA & 40.65 & 45.12 & & 42.67 \\
\hline C16:1n-7 & 2.70 & 2.78 & 6.06 & 2.11 \\
\hline C16:1n-9 & 0.40 & & 0.43 & \\
\hline C18:1n-9 & 26.19 & 31.10 & 27.50 & 26.46 \\
\hline $\begin{array}{l}\text { C20:n-9 } \\
\text { total }\end{array}$ & 0.55 & 0.11 & 0.10 & 0.12 \\
\hline MUFA & 33.71 & 34.41 & & 31.20 \\
\hline C18:2n-6 & & & & \\
\hline LA & 17.75 & 17.56 & 10.23 & 20.96 \\
\hline $\begin{array}{l}C 20: 3 n-6 \\
C 20: 4 n-6\end{array}$ & 0.44 & 0.42 & 0.47 & 0.34 \\
\hline $\mathbf{A A}$ & 0.42 & 0.48 & 1.06 & 0.48 \\
\hline total n-6 & 20.28 & 19.60 & 12.47 & 21.87 \\
\hline $\begin{array}{l}\text { C18:3n-3 } \\
\text { ALA } \\
\text { C20:5n-3 }\end{array}$ & 1.14 & 0.48 & 1.90 & 1.54 \\
\hline $\begin{array}{l}\text { EPA } \\
\text { C22:6n-3 }\end{array}$ & 0.09 & 0.11 & 0.20 & 0.08 \\
\hline DHA & 0.43 & 0.14 & 0.69 & 0.09 \\
\hline total n-3 & 2.05 & 0.85 & 3.44 & 2.11 \\
\hline
\end{tabular}


2. Fatty acids in human milk found in countries in Europe

\begin{tabular}{|c|c|c|c|c|c|c|c|c|c|c|}
\hline Europe & UK & Sweden & Germany & Spain & Portugal & Italy & France & Croatia & Serbia & Hungary \\
\hline Author & Yuhas & Zetterstrom & Genzel-Boroviczeny & Lopez-Lopez & Ribeiro & Serra & Pugo-Gunsan & Kresic & Arsic & Dekci \\
\hline lactation stage & mature & $1^{\text {st }}$ month & $30^{\text {th }}$ day & $>2$ weeks & 4 weeks & $14,21,28$ day & $42^{\text {th }}$ day & 3 months & $14^{\text {th }}$ day & mature \\
\hline C12:0 & 4.99 & 3.56 & & 6.28 & 4.55 & & 5.51 & & 6.48 & \\
\hline C14:0 & 5.87 & 5.98 & 7.39 & 6.00 & 5.36 & 10.69 & 7.49 & & 7.44 & \\
\hline C16:0 & 22.59 & 25.19 & 22.76 & 19.48 & 22.14 & 29.26 & 25.93 & & 22.24 & \\
\hline C18:0 & 6.25 & 1.17 & 7.47 & 6.25 & 5.93 & 5.55 & 7.09 & & 6.45 & \\
\hline total SFA & & 42.94 & & 40.66 & 40.42 & 45.50 & 48.23 & 32.60 & 44.30 & 39.79 \\
\hline C16:1n-7 & 2.85 & & & 1.78 & 0.10 & & 2.57 & & & \\
\hline C16:1n-9 & 0.44 & 2.69 & & 0.42 & & & & & 2.50 & \\
\hline C18:1n-9 & 33.28 & 39.90 & 30.74 & 36.35 & 27.34 & 39.93 & 33.19 & 43.00 & 32.78 & \\
\hline C20:n-9 & 0.44 & 0.64 & & 0.60 & 0.40 & & 0.41 & & 1.04 & \\
\hline total MUFA & 40.78 & 43.74 & & 39.63 & 33.23 & 42.69 & 36.78 & & 36.56 & 39.26 \\
\hline C18:2n-6 LA & 10.45 & 10.29 & 11.81 & 16.59 & 16.20 & 9.79 & 11.81 & 16.30 & 16.29 & 15.23 \\
\hline$C 20: 3 n-6$ & 0.33 & 0.37 & 0.41 & 0.48 & 0.45 & 0.25 & 0.25 & & 0.57 & 0.35 \\
\hline C20:4n-6 AA & 0.36 & 0.42 & 0.46 & 0.53 & 0.52 & 0.47 & 0.24 & 0.40 & 0.51 & 0.51 \\
\hline total n-6 & 12.42 & 11.67 & & 18.33 & 18.09 & 11.08 & 12.64 & 18.20 & 18.24 & \\
\hline C18:3n-3 ALA & 1.22 & 1.25 & 0.74 & 0.75 & 0.66 & 0.36 & 0.55 & 1.40 & 0.49 & 0.70 \\
\hline C20:5n-3 EPA & 0.11 & 0.06 & 0.12 & 0.10 & 0.16 & 0.09 & 0.03 & 0.10 & & 0.04 \\
\hline C22:6n-3 DHA & 0.24 & 0.28 & 0.32 & 0.15 & 0.42 & 0.12 & 0.14 & 0.20 & 0.19 & 0.22 \\
\hline total $n-3$ & 1.94 & 1.64 & & 1.38 & 1.42 & 0.74 & 1.05 & 1.70 & 0.86 & \\
\hline
\end{tabular}


3. Fatty acids in human milk found in countries in Africa and Oceania.

\begin{tabular}{|c|c|c|c|}
\hline Africa & $\begin{array}{c}\text { Nigeria } \\
\text { rural }\end{array}$ & $\begin{array}{c}\text { Nigeria } \\
\text { urban }\end{array}$ & Mauritus \\
\hline Author & Glew & Glew & $\begin{array}{c}\text { Pugo- } \\
\text { Gunsan }\end{array}$ \\
\hline lactation stage & & & 42nd day \\
\hline $\mathbf{N}$ & 41 & 41 & 13 \\
\hline C12:0 & 10.10 & 10.01 & 7.19 \\
\hline C14:0 & 13.50 & 13.5 & 6.96 \\
\hline C16:0 & 25.00 & 25.3 & 18.51 \\
\hline C18:0 & 4.50 & 5 & 4.97 \\
\hline total SFA & 57.10 & 56.52 & 40.21 \\
\hline C16:1n-7 & 2.00 & 1.7 & 1.95 \\
\hline C16:1n-9 & 0.07 & 0.06 & \\
\hline C18:1n-9 & 27.90 & 29.2 & 23.01 \\
\hline C20:n-9 & & & 0.22 \\
\hline total MUFA & & & 25.56 \\
\hline C18:2n-6 LA & 7.80 & 7 & 26.85 \\
\hline$C 20: 3 n-6$ & 0.13 & 0.11 & 0.46 \\
\hline C20:4n-6 AA & 0.62 & 0.48 & 0.48 \\
\hline total n-6 & & & 28.55 \\
\hline C18:3n-3 ALA & 0.80 & 0.77 & 2.65 \\
\hline C20:5n-3 EPA & 0.07 & 0.08 & 0.12 \\
\hline C22:6n-3 DHA & 0.15 & 0.4 & 0.38 \\
\hline total n-3 & & & 3.65 \\
\hline
\end{tabular}

\begin{tabular}{|l|cc|}
\hline Oceania & Australia & Australia \\
\hline Author & Yuhas & Gibson \\
\hline lactation stage & & 30th day \\
N & 48 & 23 \\
\hline C12:0 & 5.49 & \\
C14:0 & 6.28 & \\
C16:0 & 22.26 & \\
C18:0 & 6.77 & \\
total SFA & 43.79 & 47.21 \\
& & \\
C16:1n-7 & 2.97 & \\
C16:1n-9 & 0.42 & \\
C18:1n-9 & 32.23 & \\
C20:n-9 & 0.38 & \\
total MUFA & & 36.48 \\
C18:2n-6 LA & 10.66 & 13.56 \\
C20:3n-6 & 0.31 & 0.44 \\
C20:4n-6 AA & 0.38 & 0.45 \\
total n-6 & 12.74 & \\
C18:3n-3 ALA & 0.90 & 0.89 \\
C20:5n-3 EPA & 0.10 & 0.07 \\
C22:6n-3 DHA & 0.23 & 0.26 \\
total n-3 & 2.28 & \\
\hline
\end{tabular}




\section{Fatty acids in human milk found in countries in Asia and South Asia.}

\begin{tabular}{|c|c|c|c|}
\hline Asia & China & $\begin{array}{l}\text { North } \\
\text { China }\end{array}$ & Japan \\
\hline Author & Yuhas & Wan & Yuhas \\
\hline $\begin{array}{l}\text { lactation stage } \\
\mathbf{N}\end{array}$ & $\begin{array}{c}\text { mature } \\
50 \\
\end{array}$ & $\begin{array}{c}9-12 \\
\text { week } \\
52 \\
\end{array}$ & $\begin{array}{c}\text { mature } \\
51 \\
\end{array}$ \\
\hline C12:0 & 4.24 & 4.71 & 5.86 \\
\hline C14:0 & 3.61 & 3.92 & 6.11 \\
\hline C16:0 & 18.62 & 18.68 & 20.20 \\
\hline C18:0 & 6.13 & 5.63 & 6.14 \\
\hline total SFA & 35.13 & 35.92 & 41.51 \\
\hline C16:1n-7 & 1.88 & 1.29 & 2.56 \\
\hline C16:1n-9 & 0.49 & & 0.36 \\
\hline C18:1n-9 & 36.49 & 31.26 & 31.43 \\
\hline C20:n-9 & 1.25 & & 0.52 \\
\hline total MUFA & 43.90 & 32.59 & 38.43 \\
\hline C18:2n-6 LA & 14.88 & 17.73 & 12.66 \\
\hline C20:3n-6 & 0.28 & 0.23 & 0.25 \\
\hline C20:4n-6 AA & 0.49 & 0.30 & 0.40 \\
\hline total n-6 & 16.72 & 18.75 & 14.42 \\
\hline C18:3n-3 ALA & 2.02 & 1.03 & 1.33 \\
\hline C20:5n-3 EPA & 0.07 & & 0.26 \\
\hline C22:6n-3 DHA & 0.35 & 0.19 & 0.99 \\
\hline total $n-3$ & 2.90 & 1.22 & 3.10 \\
\hline
\end{tabular}

\begin{tabular}{|c|c|c|c|}
\hline South Asia & Phillipines & $\begin{array}{c}\text { West } \\
\text { India } \\
\text { Urban }\end{array}$ & Nepal \\
\hline Author & Yuhas & Roy & Glew \\
\hline lactation stage & mature & $\begin{array}{l}\text { 2nd-3th } \\
\text { week }\end{array}$ & $\begin{array}{c}\text { 2nd-4th } \\
\text { week }\end{array}$ \\
\hline $\mathbf{N}$ & 54 & 62 & 48 \\
\hline C12:0 & 13.82 & 2.43 & 12.00 \\
\hline C14:0 & 12.12 & 3.67 & 10.50 \\
\hline C16:0 & 23.02 & 20.48 & 21.70 \\
\hline C18:0 & 4.75 & 3.85 & 4.88 \\
\hline total SFA & 57.06 & 35.13 & 52.60 \\
\hline C16:1n-7 & 4.59 & 2.64 & 2.76 \\
\hline C16:1n-9 & 0.08 & & \\
\hline C18:1n-9 & 21.85 & 28.35 & 25.50 \\
\hline C20:1n-9 & 0.28 & 0.84 & 2.22 \\
\hline total MUFA & 30.33 & 34.03 & 34.40 \\
\hline C18:2n-6 LA & 7.90 & 11.05 & 7.91 \\
\hline C20:3n-6 & 0.31 & 0.65 & 0.31 \\
\hline C20:4n-6 AA & 0.39 & 0.65 & 0.35 \\
\hline total n-6 & 9.47 & 13.59 & 10.00 \\
\hline C18:3n-3 ALA & 0.43 & 1.68 & 1.93 \\
\hline C20:5n-3 EPA & 0.15 & 0.39 & 0.13 \\
\hline C22:6n-3 DHA & 0.74 & 0.72 & 0.21 \\
\hline total n-3 & 1.71 & 3.65 & 2.44 \\
\hline
\end{tabular}


5. Fatty acids in human milk found in countries in Middle East.

\begin{tabular}{|c|c|c|c|c|}
\hline Middle east & West Iran & Iraq Suburban & Israel & Turkey \\
\hline Author & Bahrami & Al-Tamer & Saphier & Aydin \\
\hline $\begin{array}{l}\text { lactation stage } \\
\mathbf{N}\end{array}$ & 52 & $\begin{array}{c}5 \text { th mo } \\
25\end{array}$ & $\begin{array}{c}\text { 2nd-3rd mo } \\
29\end{array}$ & $\begin{array}{c}28 \text { th day } \\
15\end{array}$ \\
\hline $\begin{array}{l}\text { C12:0 } \\
\text { C14:0 } \\
\text { C16:0 } \\
\text { C18:0 } \\
\text { total SFA }\end{array}$ & 41.30 & $\begin{array}{c}9.67 \\
14.97 \\
22.09 \\
5.23 \\
55.71\end{array}$ & $\begin{array}{l}21.40 \\
43.00\end{array}$ & $\begin{array}{c}6.91 \\
9.60 \\
23.05 \\
8.45 \\
51.56\end{array}$ \\
\hline $\begin{array}{l}\text { C16:1n-7 } \\
\text { C16:1n-9 }\end{array}$ & & 3.09 & & 1.64 \\
\hline $\begin{array}{l}\text { C18:1n-9 } \\
\text { C20:1n-9 } \\
\text { total MSFA }\end{array}$ & 30.90 & $\begin{array}{l}23.53 \\
29.62\end{array}$ & $\begin{array}{l}31.30 \\
33.50\end{array}$ & $\begin{array}{c}13.31 \\
0.86 \\
17.17\end{array}$ \\
\hline $\begin{array}{l}\text { C18:2n-6 LA } \\
\text { C20:3n-6 } \\
\text { C20:4n-6 AA } \\
\text { total n-6 }\end{array}$ & $\begin{array}{l}13.80 \\
1.40\end{array}$ & $\begin{array}{c}11.55 \\
0.16 \\
11.79\end{array}$ & $\begin{array}{l}20.00 \\
0.44\end{array}$ & $\begin{array}{c}25.58 \\
0.63 \\
1.81 \\
29.43\end{array}$ \\
\hline $\begin{array}{l}\text { C18:3n-3 ALA } \\
\text { C20:5n-3 EPA } \\
\text { C22:6n-3 DHA } \\
\text { total n-3 }\end{array}$ & $\begin{array}{l}1.10 \\
0.20\end{array}$ & $\begin{array}{l}0.20 \\
0.06 \\
0.12 \\
0.38\end{array}$ & $\begin{array}{l}2.00 \\
0.17\end{array}$ & $\begin{array}{l}1.20 \\
0.08 \\
0.52 \\
1.85\end{array}$ \\
\hline
\end{tabular}




\section{VANESA MENDEZ}

Born, Lima, Peru

B.S., Chemistry

Pontificia Universidad Catolica del Peru

Lima, Peru

2011

M.S., Chemistry

Florida International University

Miami, Florida

2012

Funding: Grants-In-Aid of Research.

Sigma Xi, The Scientific Research Society

2014 Doctoral Candidate

Florida International University

Miami, Florida

2014

2nd Place Student Presentation Award

17th International Symposium on Carotenoids.

Salt Lake City, Utah

2015

Funding: Student Summer Research Award Biomedical

Research Initiative. Florida International University

\section{PUBLICATIONS}

Mendez, V. Measurement of Carotenoid Levels in Human Serum and a Catalog of the Lutein Conformation Populations from Semiempirical Calculations. Master's Thesis. Florida International University, Miami, October, 2011.

John Landrum, Richard Bone, Vanesa Mendez, Anisley Valenciaga and Darwin Babino. Comparison of dietary supplementation with lutein diacetate and lutein: a pilot study of the effects on serum and macular pigment. Acta. Biochim. Pol. 2012;59(1): 167-169.

Emily R. Schenk, Vanesa Mendez, John T. Landrum, Mark E. Ridgeway, Melvin A. Park, and Francisco Fernandez-Lima. Direct Observation of Differences of Carotenoid Polyene Chain cis/trans Isomers Resulting from Structural Topology. Anal. Chem., 2014, 86 (4), pp 2019-2024. 